\title{
Review Article \\ Heat Transfer Augmentation Technologies for Internal Cooling of Turbine Components of Gas Turbine Engines
}

\author{
Phil Ligrani \\ Parks College of Engineering, Aviation, and Technology, Saint Louis University, 3450 Lindell Boulevard, \\ McDonnell Douglas Hall Room 1033A, St. Louis, MO 63103, USA
}

Correspondence should be addressed to Phil Ligrani; p_ligrani@msn.com

Received 24 September 2012; Revised 7 January 2013; Accepted 23 January 2013

Academic Editor: J.-C. Han

Copyright (C) 2013 Phil Ligrani. This is an open access article distributed under the Creative Commons Attribution License, which permits unrestricted use, distribution, and reproduction in any medium, provided the original work is properly cited.

To provide an overview of the current state of the art of heat transfer augmentation schemes employed for internal cooling of turbine blades and components, results from an extensive literature review are presented with data from internal cooling channels, both with and without rotation. According to this survey, a very small number of existing investigations consider the use of combination devices for internal passage heat transfer augmentation. Examples are rib turbulators, pin fins, and dimples together, a combination of pin fins and dimples, and rib turbulators and pin fins in combination. The results of such studies are compared with data obtained prior to 2003 without rotation influences. Those data are comprised of heat transfer augmentation results for internal cooling channels, with rib turbulators, pin fins, dimpled surfaces, surfaces with protrusions, swirl chambers, or surface roughness. This comparison reveals that all of the new data, obtained since 2003, collect within the distribution of globally averaged data obtained from investigations conducted prior to 2003 (without rotation influences). The same conclusion in regard to data distributions is also reached in regard to globally averaged thermal performance parameters as they vary with friction factor ratio. These comparisons, made on the basis of such judgment criteria, lead to the conclusion that improvements in our ability to provide better spatiallyaveraged thermal protection have been minimal since 2003. When rotation is present, existing investigations provide little evidence of overall increases or decreases in overall thermal performance characteristics with rotation, at any value of rotation number, buoyancy parameter, density ratio, or Reynolds number. Comparisons between existing rotating channel experimental data and the results obtained prior to 2003, without rotation influences, also show that rotation has little effect on overall spatially-averaged thermal performance as a function of friction factor.

\section{Introduction}

The techniques employed to enhance convective heat transfer rates for internal cooling of turbine airfoils of gas turbine engines include rib turbulators, pin fins, dimpled surfaces, surfaces with arrays of protrusions, swirl chambers, and surface roughness [1]. According to Ligrani et al. [1], all of these devices act to increase secondary flows and turbulence levels to enhance mixing, in some cases, to form coherent fluid motions in the form of streamwise-oriented vortices. Such vortices and secondary flows not only act to increase secondary advection of heat away from surfaces, but also to increase three-dimensional turbulence production by increasing shear and creating gradients of velocity over significant flow volumes. These then give larger magnitudes of turbulence transport over larger portions of the flow fields. All of the devices mentioned also provide some heat transfer augmentation by increasing surface areas for convective heat transfer. The overall objective for such internal cooling technologies is optimal thermal protection with minimal use of coolant air with coolant mass flow rates as small as possible, and minimal pressure drop penalties within coolant passages. Appropriate levels of thermal protection are quantified by sufficiently low absolute temperature levels, as well as relatively uniform temperature distributions, as characterized by sufficiently small temperature gradients within solid components.

Figure 1 shows a typical internal cooling arrangement for a multipass turbine blade from Han et al. [2,3]. With such an arrangement, the cooling air enters the airfoil through the 


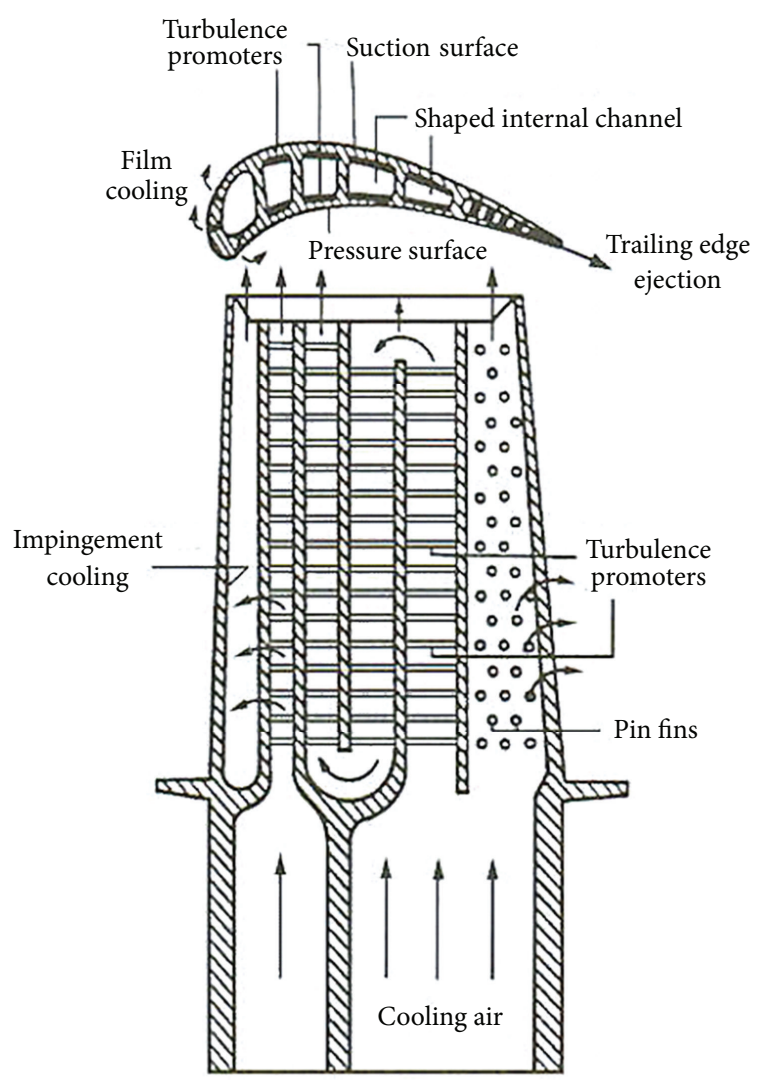

FIgURE 1: Typical internal cooling arrangement for a multipass turbine blade from Han et al. $[2,3]$.

blade root and then passes through one of several internal passages. As a result of the blade shape, the cooling passages at many locations are generally confined, and are therefore, difficult to cool. Such cooling passages also generally utilize complex cross-sectional shapes, because they must be compatible with the external contours of the turbine airfoils. Features that provide the highest heat transfer coefficients for a given flow rate are required in the leading and trailing edge cavities of airfoils that may or may not be film cooled. The leading edge geometry is characterized by a small coolant metal area to hot gas metal area ratio, while a thin trailing edge creates geometric constraints on passage sizes and accessibility for cooling air. For this reason, impingement cooling and swirl chambers are typically employed near the leading edge of the airfoil, either together or individually, and pin fins, and more complex rib turbulator shapes are typically found near the trailing edge regions of the airfoil. Rib turbulators, or turbulence promoters, are also located in passages near the mid-chord parts of the airfoil. Dimples and/or shaped roughness elements are also sometimes employed in all interior parts of airfoils, either by themselves or in conjunction with other devices. After passing over or through these devices, the cooling air then either exits through film cooling holes, or though exit passages which lead to another part of the airfoil.
Numerous and important constraint requirements are imposed upon the development of internal cooling configurations for gas turbine engines, such as the arrangement presented in Figure 1. This is a consequence of the complexity and sophistication of turbine component design. Satisfying these constraints requires simultaneous consideration of a variety of multidisciplinary technological issues. These include manufacturing, available volume space, airfoil shape, airfoil surface cooling requirements, aerodynamic losses, coolant availability, second law losses, and pressure drop penalties, in addition to heat transfer and thermal considerations. As a result, the current trend for advanced turbine component cooling design is a simultaneous development of external thermal protection schemes, like film cooling, with internal cooling technologies, including consideration of the adjacent conjugate conduction variations in adjacent solid components, as well as associated aerodynamic pressure loss penalty issues. One way that designers, manufacturers, and researchers attempt to meet these myriad requirements is development of internal cooling technologies which employ combinations of different devices together within one coolant passage. Examples include Murata et al. [4] who employ rib turbulators, pin fins, and dimples together, Rao et al. [5] who utilize a combination of pin fins, and dimples, and Siw et al. [6] who use rib turbulators and pin fins in combination within the same coolant passage.

One key objective of the present paper is discussion of new internal passage heat transfer augmentation technologies, such as these, as they are employed for internal cooling of turbine components of gas turbine engines. The overall paper is presented in three major parts. Presented first is a brief review of developments up to 2003, considering investigations which do not include the effects of rotation. An overview of more recent developments since 2003 (without rotation issues considered) is presented next. The third and final part of the paper then addresses the effects of rotation on heat transfer augmentation technologies for internal cooling.

Included within the discussions are comparisons of heat transfer augmentations, friction factor augmentations, and thermal performance parameters provided by different devices used for internal cooling of turbine airfoils, including rib turbulators, pin fins, dimpled surfaces, surfaces with arrays of protrusions, swirl chambers, and surface roughness. To characterize the heat transfer augmentations, $\mathrm{Nu} / \mathrm{Nu}_{o}$, the ratio of the Nusselt number of the channel with augmenters divided by the Nusselt number of a similar smooth channel (at the same experimental conditions) is employed. Likewise, the increase in pressure losses is represented by $f / f_{o}$, the ratio of the friction factor measured in a channel with the augmenters divided by the friction factor of a similar smooth channel at the same experimental conditions. Such Nusselt number and friction factor ratios provide means to compare different techniques, as well as the performance of a given technique at different experimental conditions.

The overall intent of the discussions and presentation is development of improved cooling configurations, which lead to overall improvements in overall gas turbine engine efficiency, reliability, and durability. In addition, the area is important because schemes for internal cooling of turbine 
airfoils can also be used in other devices, such as combustion chambers, air-conditioners, radiators, microscale heat exchangers, macroscale heat exchangers, devices for electronics cooling, and biomedical devices, to name only a few.

\section{Developments up to 2003}

Within this part of the paper, heat transfer augmentation technologies developed prior to 2003, generally without consideration of rotation effects, are briefly summarized. The discussion is presented in five parts: pin fin arrays, dimpled surfaces, rib turbulators, swirl chambers, and overall comparisons.

2.1. Pin Fin Arrays. When employed for internal cooling, pin fins or pedestals are generally arranged into arrays and extend between two opposite walls of an internal cooling passage. Brigham and VanFossen [7] and Ligrani and Mahmood [8] describe typical arrangements. According to Ligrani et al. [1], pin fins are generally used in the parts of turbine airfoils where higher levels of heat transfer augmentation are required and where high pressure drops are tolerated, and in many cases, even desired. Trailing edges of airfoils fall into this category. High coolant Reynolds numbers, associated with high coolant pressure levels, are desirable for heat transfer. However, by the time the coolant reaches the trailing edge, it has often acquired a significant amount of heat, and the process of extracting heat from trailing edge components is more challenging compared to other parts of the airfoil. At the same time, manufacturing limitations on the width of trailing edge slots prevent adequate restriction for the coolant, thus requiring that the flow is metered upstream. Pin fins or pedestals provide appropriate structural support near the trailing edge and are effective for allowing the pressure throughout most of the cooling circuit to remain high, while providing the necessary restriction near the trailing edge, to limit the coolant consumption to the desired level.

The large majority of existing pin fin investigations only focus on heat or mass transfer. Considered are the influences of pin geometry, pin materials, pin fin array configuration, channel geometry, different Reynolds numbers, and other parameters [12-15]. VanFossen [16] presents heat transfer coefficients measured on circular pin fins made of different materials, which are placed in rectangular cross-sectional channels. Brigham and VanFossen [7] report large overall heat transfer and mass transfer augmentations for circular pin fins arranged in in-line and staggered arrays. Simoneau and VanFossen [17] measure pin surface heat transfer coefficients and streamwise turbulence intensities as the test section gas temperature is changed. Later investigations by Lau et al. [18] and McMillin and Lau [19] consider streamwise pressure variations in a channel with circular pin fins. Chyu [20], Chyu and Goldstein [21], and Chyu et al. [22] report local and spatially averaged mass transfer coefficients for different pin fin arrays, which provide evidence of large overall mass transfer augmentations for certain in-line and staggered circular arrays. Grannis and Sparrow [23] numerically simulate the two-dimensional flow and pressure fields around diamondshaped pin fins. Olson [24] presents normalized pressure drop and wall Nusselt number data as dependent upon Reynolds number and wall heat flux magnitude.

The effects of different pin fin shapes on heat transfer and flow in internal passages are considered by a number of investigators $[8,20,22,23,25-30]$. A variety of pin fin shapes are investigated, including circular $[8,20,25-30]$, pins with end-wall fillets [20], diamond [23, 25-27], three-dimensional protruding elements [26], cubic $[25,26]$, and elliptical [28, 29]. Of these studies, the one described by Hwang and $\mathrm{Lu}$ [27] is especially unique because three pin fin configurations, each arranged in a staggered array, are considered in a trapezoidal duct, both with and without lateral flow ejection.

In regard to pin fin flow structure, Chyu and Natarajan [26] present mass transfer distributions and surface flow streakline patterns (obtained using an oil-graphite technique) around several different shapes of single protruding elements placed on an endwall, including a cylinder, a cube, a diamond, a pyramid, and a hemisphere. The vortices and mixing present just downstream of pin fins are further elucidated by results presented by Uzol and Camci [29]. According to Won et al. [30], the most important features downstream of an individual pin are two large vortex pairs, which are formed by the blockage effects of the pin fins. The secondary flows within and around these vortices are especially intense relative to other secondary flows which are present. Additional description of the different flow structures which develop near one endwall junction of one pin fin (as it is contained within a pin array) is provided by Ligrani et al. [1], including a typical surface Nusselt number ratio distribution, produced on the endwall by these flow structures [8].

2.2. Dimpled Surfaces. Dimples are arrays of indentations along surfaces. These are most commonly spherical in shape, although a variety of other shapes have also been employed, including triangular and tear-drop. Arrays of dimples are beneficial method for internal cooling because they produce multiple vortex pairs which augment local Nusselt number distributions as they advect downstream. They are notable for the low pressure drop penalties which they produce, which is because they do not protrude into the flow to produce significant amounts of form drag. With this benefit, dimples offer advantages for cooling later turbine stages where lower pressure cooling air is employed. They are also advantageous because the pressure drop which they produce through an airfoil passage is relatively low, which allows favorable pressure margins to be maintained in parts of the airfoil interior which are further downstream [1]. Another benefit is dimple manufacture removes material from internal cooling passage components, in contrast to rib turbulators and pin fins, which require addition of extra material and weight.

One of the first published studies of the effects of hemispherical dimples on flow structure and heat transfer is described by Murzin et al. [31]. These investigators describe the flow over and within shallow spherical depressions and conclude that this flow is mostly symmetric, and consists of a stable recirculatory flow inside of the depression. Gromov 
et al. [32] describe symmetric and nonsymmetric streamlines and flow patterns produced by hemispherical cavities with a variety of sizes. Cells of fluid motion are described in the form of tightening spirals, helical streamlines, and horseshoe shaped vortices. Kesarev and Kozlov [33] present distributions of local heat transfer coefficients inside a single hemispherical cavity, and indicate that the convective heat transfer from the cavity is higher, especially on the downstream portion, than that from the surface of a plane circle of the same diameter as the cavity diameter. Afanasyev et al. [34] describe the heat transfer enhancement mechanism for flows over walls indented with regular arrays of spherical pits with several different shapes. Enhancements of 30-40 percent, with pressure losses that are not increased appreciably relative to a smooth surface, are reported. Belen'kiy et al. [35] describe heat transfer intensification from a tube surface fitted with a staggered array of concave dimples on surfaces annular internal passages. Terekhov et al. [36] present experimental measurements of flow structure, pressure fields, and heat transfer in a channel with a single dimple on one surface. Different magnitudes and frequencies of flow oscillations are described, along with heat transfer and pressure loss dependence on dimple geometry. From flow visualizations, Zhak [37] describes a variety of vortex structures in different shaped rectangular cavities. Schukin et al. [38] present results which are directly applicable to gas turbine cooling, including average heat transfer coefficients from the measurements on a heated plate downstream of a single hemispherical cavity in a diffuser channel and in a convergent channel. Bearman and Harvey [39] investigate cross flows over cylindrical surfaces with an array of dimples. The geometry of the dimples corresponds to the optimum concavity depth found by Kimura and Tsutahara [40] for minimum drag on cylinders. At high Reynolds numbers, significantly lower drag coefficients are present with arrays of dimples compared to smooth surfaces. Kithcart and Klett [41] compare heat transfer and skin friction of flows over surfaces on one wall of a rectangular channel with hemispherical dimples, hemispherical protrusions, and rectangular protrusions. The authors conclude that all three geometries produce similar levels of heat transfer augmentation.

More recently, Chyu et al. [42] present data showing the effects of Reynolds number on local heat transfer coefficient distributions on surfaces imprinted with staggered arrays of two different shapes of concavities: spherical and tear-drop. Their measurements show distributions of heat transfer coefficients everywhere on surfaces which are significantly higher than values in channels with smooth surfaces. Lin et al. [43] present computational simulation results of the flow structure and resulting heat transfer distributions for the same surface geometries and flow conditions. Flow streamlines and temperature distributions are presented which provide insight into the flow structural characteristics produced by the dimples. Another study, described by Gortyshov et al. [44], employs spherically shaped dimples, called spherical intensifiers, placed at different relative positions on the opposite sides of a narrow channel. The investigators observe that an increase in relative dimple depth produces increases in surface heat transfer rates, as well as increases in streamwise pressure penalties. Moon et al. [45] give heat transfer and friction factor data, for ratios of channel height to dimple print diameter from 0.37 to 1.49 , that illustrate the effects of channel height on a surface with a staggered pattern of dimples. According to these investigators, improvements in heat transfer intensification and pressure losses remain at approximately constant levels over the ranges of Reynolds number and channel height investigated. Chen et al. [46] employ hemispherical-shaped protrusions on the insides of tubes to augment surface heat transfer rates. Six different protrusion arrangements are employed, which increase surface heat transfer rates above those present in smooth tubes by 25 to 137 percent when compared at the same Reynolds number. Data from Moon and Lau [47] are based on measurements made only at a few discrete locations on their test surface, whereas the other studies obtained results from averages made from spatially-resolved results, measured over continuous sections of test surfaces.

Mahmood et al. [48] and Ligrani et al. [1] describe the mechanisms responsible for local and spatially averaged heat transfer augmentations on flat channel surfaces with an array of dimples along one wall. In the Mahmood et al. [48] study, the ratio of channel height to dimple print diameter is 0.5 . Key flow features responsible for local heat transfer augmentations include (i) shedding of multiple vortex pairs from dimples, (ii) the strong secondary fluid motions within these vortex pairs and associated vortical fluid, (iii) shear layer reattachment within each dimple, and (iv) periodicity and unsteadiness associated with vortex pair shedding and the flows within individual dimples, which is produced as flow is ejected and then inrushes to each dimple. This unsteadiness causes the thermal boundary layer which forms downstream of individual dimples to be periodically reinitialized, and increases flow mixing over length-scales approximately equal to the dimple print diameter. The effects on thermal transport are especially pronounced near downstream rims of dimples as well as on flat surfaces downstream of and between dimples [1]. Mahmood and Ligrani [49] consider the effects of the ratio of inlet stagnation temperature to local surface temperature for ratios of channel height to dimple print diameter of $0.20,0.25,0.50$, and 1.00. According to these authors, visualized smoke patterns show that vortex pairs, which are periodically shed from the dimples, become stronger as the ratio of channel height to print diameter decreases. However, regardless of the ratio of channel height to dimple print diameter, local Nusselt numbers increase substantially as the ratio of inlet stagnation temperature to local surface temperature decreases.

Burgess and Ligrani [51] present spatially resolved Nusselt numbers measured on the dimpled test surface placed on one wall of a channel with a height to dimple print diameter ratio of 1.0, and ratios of dimple depth to dimple print diameter of $0.1,0.2$, and 0.3 . The opposite channel wall is smooth. Reynolds number based on channel height for the measurements is 20,000. According to these investigators, lower Nusselt number ratios are located over the upstream halves of the depressions. Local Nusselt number ratios are then higher in the downstream halves. The highest values are then located near the downstream rims of each dimple, both 
slightly within each depression, and on the flat surface just downstream of each dimple. In general, spatially averaged Nusselt number augmentations from dimples increase (at a particular Reynolds number), as the ratio of dimple depth to dimple print diameter increases from 0.1 to 0.3 . However, in application to component internal cooling, a limit often exists regarding maximum allowable manufactured dimple depth, and thus, dimple depth relative to the thickness of associated turbine blade and vane material is an important consideration. Consistent with the results of Kesarev and Kozlov [33] and Schukin et al. [38], most local values in the concave cavities are higher than values measured a smooth channel at the same Reynolds number and temperature ratio. Additional information on the flow structure due to dimple depressions on a channel surface is provided by Ligrani et al. [52]. Here, detailed descriptions of the behavior of the primary and secondary vortex pairs, which are shed from the dimples, are described. Most importantly, the locations of the primary and secondary vortex pairs coincide closely with locations where the normalized longitudinal Reynolds normal stress is increased. Frequent, continuous, unsteady interactions are also described between the flows in adjacent dimples, where the frequency of the large-scale unsteadiness scales on time-averaged bulk velocity and dimple print diameter.

Mahmood et al. [53] and Ligrani et al. [54] consider heat transfer and flow structure in a channel with dimples and protrusions on opposite walls. Similar arrangements, but only with measurements of friction factors and some flow structural characteristics, are considered by Kithcart and Klett [41]. According to Ligrani et al. [54], instantaneous flow visualization images and surveys of time-averaged flow structure show that the protrusions result in added vortical, secondary flow structures, and flow mixing. As a result, local friction factors and local Nusselt numbers are augmented compared to a channel with no protrusions on the top wall. Mahmood et al. [53] indicate that important Nusselt number variations are observed as the array of protrusions is changed with respect to the locations of the dimples. With protrusions, form drag and channel friction are increased. As a result, thermal performance parameters are then generally slightly lower when protrusions and dimples are employed, compared to a channel with a smooth-dimple arrangement. Additional information on the flow structure resulting from an array of surface dimples is provided by Ligrani et al. [1]. According to Isaev et al. [55], the flow inside of a spherical indentation dimple is also quite complex, with nonsymmetric flow patterns sometimes present, even though the dimple is symmetric. According to these investigators, "large-scale vortex rings, inside which particles perform reciprocating motions are incorporated into the spiral flows outgoing from singularities at the surface of the well."

2.3. Rib Turbulators. Rib turbulators are often in the form of rectangular cross-sectional bars mounted along the surface, which are often angled with respect to the bulk flow direction. Because they protrude into the flow, they act to trip the flow, mix the flow, and also generate vortices and threedimensional velocity gradients. Rib turbulators or trip strips are general purpose heat transfer augmentors in cooled airfoils. By varying the main geometric parameters, trip strip height, channel blockage, orientation, and spacing, it is possible to optimize the cooling scheme in such a way that the airfoil mid-body is not overcooled, while providing the necessary exit temperatures at the leading edge and trailing edge where film cooling may be required [1]. Due to casting limitations, ribs are rarely rectangular in crosssection when employed within turbine components. Instead, they often maintain more rounded profiles due to manufacturability limitations. A significant number of experimental and numerical studies address the effects of rib turbulators on heat transfer in internal channels $[1,56-100]$. Considered are single pass and multipass channels, square and rectangular channels, and channels with and without rotation.

Several of the earliest experimental studies consider single-pass, stationary channels with no rotation $[62,68-$ $71,78,92,93$ ]. Of these studies, Casarsa et al. [62] characterize the velocity and heat transfer fields in an internal cooling channel with $90^{\circ}$ ribs which produce 30 percent blockage. Included are heat transfer enhancement magnitudes, timeaveraged distributions of mean velocity components, and time-averaged distributions of normalized root-mean-square velocity fluctuations. Mahmood et al. [78] present spatially resolved Nusselt numbers and flow structure are for a stationary channel with an aspect ratio of 4 and angled rib turbulators inclined at $45^{\circ}$ with perpendicular orientations on two opposite surfaces. The ratio of rib height to hydraulic diameter is 0.078 , the rib pitch-to-height ratio is 10 , and the blockage provided by the ribs is 25 percent of the channel cross-sectional area. According to these authors, spatially resolved local Nusselt numbers are highest on tops of the rib turbulators, with lower magnitudes on flat surfaces between the ribs, where regions of flow separation and shear layer reattachment have pronounced influences on local surface heat transfer behavior. Also important are intense, highly unsteady secondary flows and vortex pairs, which increase secondary advection and turbulent transport over the entire channel cross-section.

Other studies of stationary channels, prior to 2003, with no rotation, consider single-pass and multipass channels. Thurman and Poinsatte [97] measure heat transfer and bulk air temperature in a three-pass duct with orthogonal ribs and bleed holes both located on one wall. According to these investigators, changing the locations of the ribs relative to the holes produces large changes to surface heat transfer coefficient distributions. Cho et al. [63] employ continuous and discrete, parallel and cross arrays of ribs in a single-pass square duct. Discrete ribs with gaps in between are found to produce more uniform heat transfer coefficient distributions than continuous ribs. According to these authors, remarkable enhancements of local and spatially averaged surface heat transfer rates are possible with rib turbulators, in spite of lowered local Nusselt numbers at certain locations along ribbed surfaces.

Computational studies of flows and heat transfer in ducts with rib turbulators consider straight single-pass ducts [56, $72,73,86,89,99]$, two-pass ducts [57], two-pass ducts with U-shaped channels in between $[60,76,84,88,90], 90^{\circ}$ 
orthogonal ribs $[72,73,84,86,99], 45^{\circ}$ angled ribs $[56,57$, $60,76,88-90], 45^{\circ} \mathrm{V}$-shaped ribs [73], and rotation [56, 57, $60,72,76,84,88,90]$. In one case [89], the angled ribs placed on two opposite walls of the channel are rounded. Jia et al. [73] and Watanabe and Takahashi [99] include experimentally measured friction factors, Nusselt numbers, and streamwise velocity fluctuations, which are compared to numerically predicted results. Rigby and Bunker [85] employ a full threedimensional Navier-Stokes equation solver to investigate the same complex trailing edge passage of a high pressure turbine blade investigated experimentally by Bunker et al. [61].

In regard to flow structure, flow recirculation patterns around a single rib are positioned just downstream of each rib, and cover a considerable portion of the ribbed wall. Each of these recirculation zones is often considered to be a potential hot spot because it is associated with locally lower surface Nusselt numbers [1,78]. Downstream of the flow recirculation zone, downstream of each rib, is a line across the surface where streamlines reattach. Weak secondary flows often form downstream of this reattachment line for $90^{\circ}$ rib turbulators [63]. With angled ribs, additional secondary flows are induced by flow skewing $[1,62]$. Additional data regarding the effects of the cross-rib arrangement on flow structure are presented by Mahmood et al. [78] for a $45^{\circ}$ rib arrangement, by Jia et al. [73] for a square cross-section with ribs on one wall, and by Watanabe and Takahashi [99] for a channel with $90^{\circ}$ rib turbulators. Data which provide quantitative detail on the recirculation zone which forms just above a rib, the recirculation zone which forms near the corner just upstream of a rib, the large recirculation zone which is present downstream of a rib, and the spanwise vortex, or second smaller separation zone, which is present in the vicinity of the downstream bottom corner of a rib are also given by Casarsa et al. [62]. Note that the sudden expansion downstream of the rib results in a large recirculation zone. A shear layer forms between this recirculating flow, and the high-speed mainstream bulk flow, located just above [62, 99]. According to Casarsa et al. [62], the shear layer reattachment line exhibits a particular shape near the lateral wall because side wall boundary layers are altered by the presence of the rib. When the boundary layer is deviated by the rib, new streamwise vorticity is generated inside of it. The flow then rolls up on itself. The result is local deformation of the reattachment line from a straight line [62].

2.4. Swirl Chambers. Swirl chambers are internal flow passages arranged with either spinning vanes, internal inserts, or inlets and outlets configured to produce large-scale swirling of the flow (relative to the chamber dimensions) generally about the principal chamber axis [105]. This large-scale swirling and the Görtler vortex pairs that are formed enhance surface heat transfer rates. Here, the heat transfer and fluid mechanics within swirl chambers are described as they are used to augment heat transfer rates for internal turbine blade cooling, an application introduced by Glezer et al. [106, 107]. Other investigations prior to 2003 employ a variety of different methods to induce large-scale swirling, generally, with an overall goal of augmentation of heat transfer rates
[105-127]. Of these investigations, Hwang and Cheng [118] employ multiple swirling jets to augment heat transfer in a triangular duct. In some cases, the highest local heat transfer enhancements are produced on a bottom surface by wall jets, whereas in others, the highest local enhancements are produced on a target wall by impingement jets. Local and spatially averaged surface Nusselt numbers are given as they vary with Reynolds number and three different jet inlet angles [118].

The configuration employed by Hedlund and Ligrani [115], Hedlund et al. [116], and Ligrani et al. [123] models an internal passage used to cool the leading edge of a turbine blade. Multiple inlets are employed at fixed locations, to induce swirling, which makes this swirl chamber geometry different from the arrangements used in many other investigations. Hedlund et al. [116] and Hedlund and Ligrani [115] show that important variations of surface Nusselt numbers and time-averaged flow characteristics are present due to arrays of Goertler vortex pairs, especially near the inlets of the swirl chambers, where Nusselt numbers are highest. Nusselt numbers then decrease and become more spatially uniform along the interior surface of the chamber as the flows advect away from each inlet. Numerous convoluted Görtler vortex pairs of different size near the concave surface of the chamber are evidenced by smoke visualizations, as well as by time-averaged surveys of flow characteristics, which show regions of positive and negative time-averaged circumferential vorticity adjacent to each other near the concave surface. Important Nusselt number variations due to the Görtler vortex pairs are evident at nearby surface locations, where higher and lower local Nusselt numbers correspond to Görtler vortex pair downwash and upwash regions, respectively.

\subsection{Overall Comparisons. Figures 2 and 3 present compar-} isons of globally averaged Nusselt number ratios $\overline{\overline{\mathrm{Nu}}} / \mathrm{Nu}_{o}$ and friction factor ratios $f / f_{o}$ for rib turbulators, pin fins, swirl chambers, dimple-smooth arrangements, dimple-protrusion arrangements, dimple-dimple arrangements, surface roughness, and smooth-walled channels. The $f / f_{o}$ abscissa scale in Figure 3 ranges from 0 to 20 (compared to 0 to 80 in Figure 2) to provide a detailed view of variations over this friction factor ratio range [1].

According to Ligrani et al. [1], of the techniques considered, swirl chambers give some of the highest levels of heat transfer augmentation when compared at the same value of friction factor ratio. In some cases, several of the rib turbulator configurations produce comparable Nusselt number ratios. However, pressure losses and friction factor ratios associated with swirl chambers and rib turbulators are also relatively high. Many of the rib turbulator configurations produce levels of heat transfer augmentation that are higher than those produced by the dimple-protrusionsmooth configurations. However, generally, the increases in pressure losses due to the rib turbulators are also larger than those associated with the dimple-protrusion-smooth configurations. $f / f_{o}$ magnitudes are as high as 71 for channels with rib turbulators, and as high as 25 for swirl chambers, 


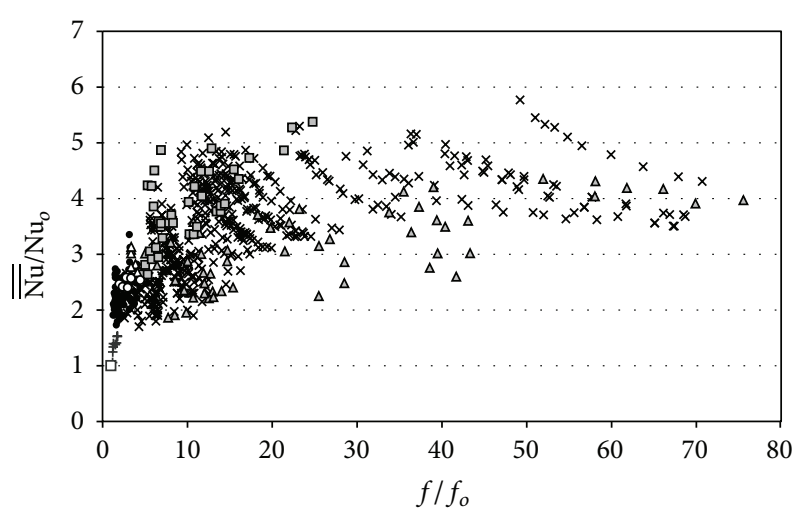

$\times$ Rib turbulators

$\Delta$ Pin fins

- Swirl chambers

- Dimple-smooth

$\Delta$ Dimple-protrusion

- Dimple-dimple

+ Surface roughness

- Smooth channel

FIgURE 2: Comparison of relative performance of rib turbulators, pin fins, swirl chambers, dimple-smooth arrangements, dimpleprotrusion arrangements, dimple-dimple arrangements, surface roughness, and smooth-walled channels from investigations prior to 2003. Globally averaged Nusselt number ratios $\overline{\overline{\mathrm{Nu}}} / \mathrm{Nu}_{o}$ are presented as dependent upon friction factor ratios $f / f_{o}[1]$.

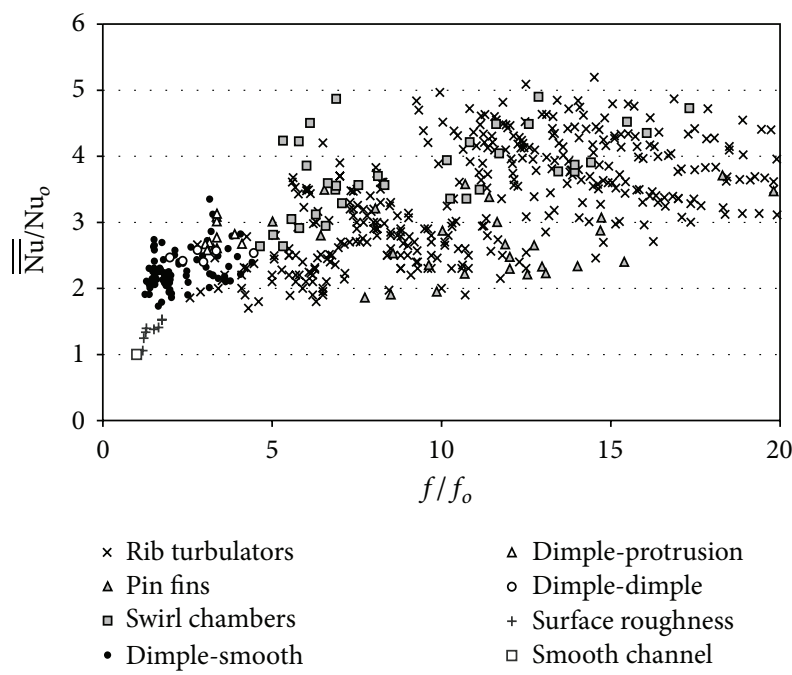

FIGURE 3: Comparison of relative performance of rib turbulators, pin fins, swirl chambers, dimple-smooth arrangements, dimpleprotrusion arrangements, dimple-dimple arrangements, surface roughness, and smooth-walled channels from investigations prior to 2003. Globally averaged Nusselt number ratios $\overline{\overline{\mathrm{Nu}}} / \mathrm{Nu}_{o}$ are presented as dependent upon friction factor ratios $f / f_{o}[1]$.

whereas magnitudes for dimple-protrusion-smooth configurations reach only up to 4.5. Nusselt number ratios in channels with pin fins lie near the bottom of or below the values for rib turbulators when compared at the same $f / f_{o}$ value. Pin fin friction factor ratios are then as high as 76 [1].

Also included in Figures 2 and 3 are Nusselt number ratios and friction factor ratios for a smooth channel, and for a channel with three-dimensional, irregular surface roughness
$[129,130]$. Such surface roughness augmenters work as microturbulators and microvortex generators. These act to increase mixing and levels of turbulence transport locally near surfaces. With this mechanism, $\overline{\overline{\mathrm{Nu}}} / \mathrm{Nu}_{o}$ range from 1.06 to 1.53 , and $f / f_{o}$ range from 1.18 to 1.75 in Figures 2 and 3 [1].

The data in these two figures are presented again in Figures 4 and 5. In the first of these figures, globally averaged thermal performance parameters $\overline{\overline{\mathrm{Nu}}} / \mathrm{Nu}_{o} /\left(f / f_{o}\right)^{1 / 3}$ are given as dependent upon the friction factor ratio $f / f_{o}$. The form of this performance parameter is suggested by Gee and Webb [131] to provide a heat transfer augmentation quantity $\left(\overline{\overline{\mathrm{Nu}}} / \mathrm{Nu}_{o}\right)$ and a friction factor augmentation quantity $\left(\left(f / f_{o}\right)^{1 / 3}\right)$, where each is given for the same ratio of mass flux in an internal passage with augmentation devices to mass flux in an internal passage with smooth surfaces. According to Figure 4 , the highest $\overline{\overline{\mathrm{Nu}}} / \mathrm{Nu}_{o} /\left(f / f_{o}\right)^{1 / 3}$ thermal performance parameters are produced by swirl chambers and dimple-smooth arrangements, and the lowest are produced by channels with arrays of pin fins. Results for rib turbulators, dimple-protrusion arrangements, dimple-dimple arrangements, surface roughness, and smooth-walled channels then generally lie between these distributions [1].

The $\overline{\overline{\mathrm{Nu}}} / \mathrm{Nu}_{o} /\left(f / f_{o}\right)$ parameter, presented in Figure 5 , is referred to as the Reynolds analogy performance parameter. The highest values of this parameter are produced by dimplesmooth arrangements, and the lowest are produced by pin fins. Results for rib turbulators, swirl chambers, dimpleprotrusion arrangements, dimple-dimple arrangements, surface roughness, and smooth-walled channels then generally lie between these distributions [1].

\section{Developments Since 2003}

Within this part of the paper, heat transfer augmentation technologies developed since 2003, generally without consideration of rotation effects, are discussed. The material is presented in five parts: pin fin arrays, dimpled surfaces and surfaces with protrusion arrays, rib turbulators, combination devices, and overall comparisons.

3.1. Pin Fin Arrays. Recent investigations of arrays of pin fins within internal ducts consider the influences of a variety geometric and flow parameters. These include pin fin shape [9], pin fin height [132], streamwise and spanwise pin-fin spacings $[133,134]$, streamwise pin fin spacing and Reynolds number [135], and combinations of pin fins with dimples [5, 136]. Also addressed in recent studies are turning flow effects on arrangements utilized for trailing edge cooling [137], high solidity pin fin arrays with incremental replenishment [138], effects of particle deposition and erosion characteristics on heat transfer in pin fin arrays [139], and effects of pin fin array detached spaces [140].

Examples from investigations of shaped pin fin arrays are shown in Figures 6 and 7 from Siw et al. [9]. Considered are triangular, semicircular, and circular pin fins. The corresponding three sets of dimensional heat transfer coefficient results are given in Figure 7 . These data sets are obtained at the 


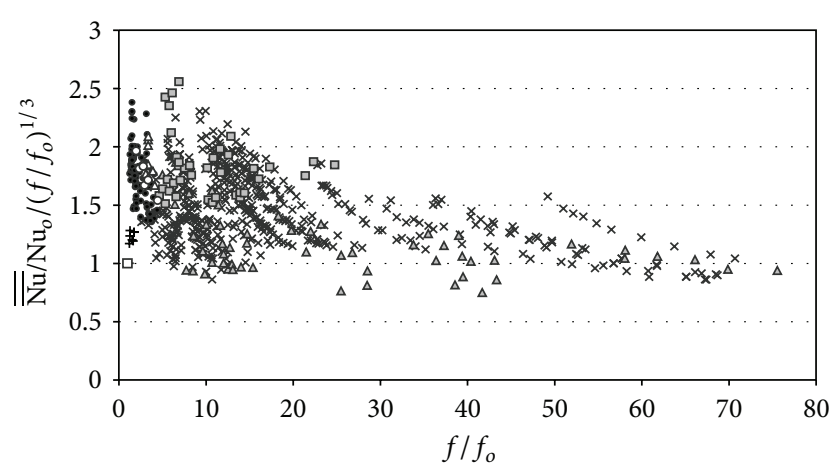

$\times$ Rib turbulators

$\triangle$ Pin fins

- Swirl chambers

- Dimple-smooth

FIGURE 4: Comparison of relative performance of rib turbulators, pin fins, swirl chambers, dimple-smooth arrangements, dimpleprotrusion arrangements, dimple-dimple arrangements, surface roughness, and smooth-walled channels from investigations prior to 2003. Globally averaged thermal performance parameters $\overline{\overline{\mathrm{Nu}}} / \mathrm{Nu}_{o} /\left(f / f_{o}\right)^{1 / 3}$ are presented as dependent upon friction factor ratios $f / f_{o}[1]$.

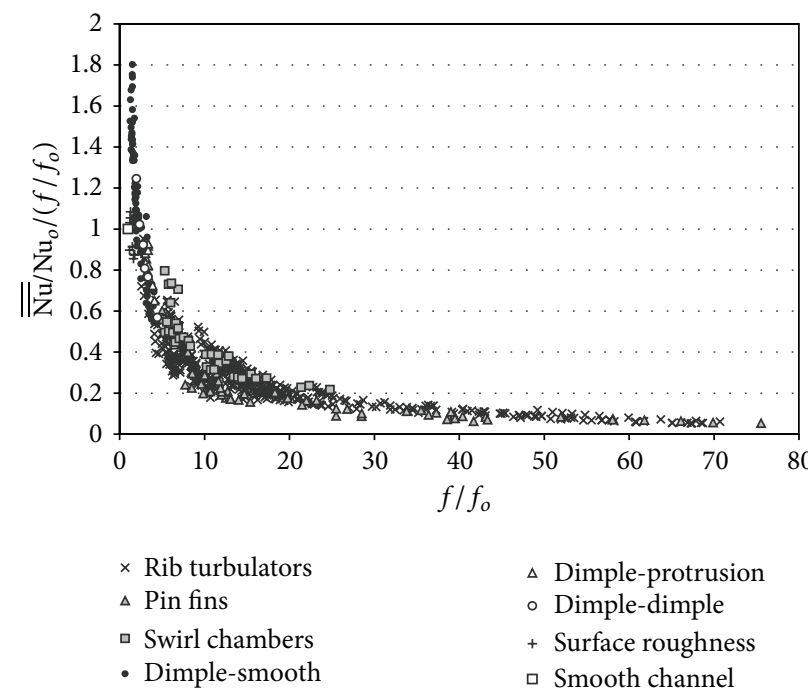

FIGURE 5: Comparison of relative performance of rib turbulators, pin fins, swirl chambers, dimple-smooth arrangements, dimpleprotrusion arrangements, dimple-dimple arrangements, surface roughness, and smooth-walled channels from investigations prior to 2003. Globally averaged thermal performance parameters $\overline{\overline{\mathrm{Nu}}} / \mathrm{Nu}_{o} /\left(f / f_{o}\right)$ are presented as dependent upon friction factor ratios $f / f_{o}[1]$.

same Reynolds number of 25,000 and with the same interpin spacing, where Reynolds number is determined using inlet duct hydraulic diameter, and bulk spatially averaged air inlet velocity. Local qualitative heat transfer coefficient variations for the circular and semicircular arrays are similar, with the highest local maxima present near the third and fourth streamwise rows, just upstream of regions where values

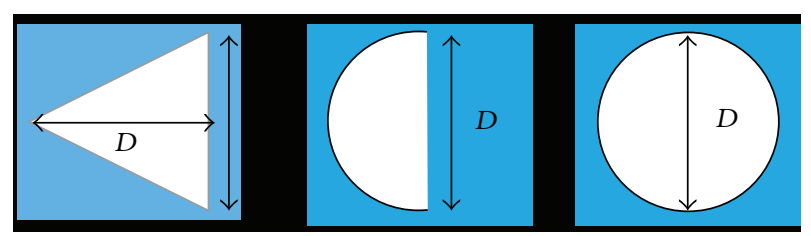

Figure 6: Pin fins element configurations employed in the investigation by Siw et al. [9].

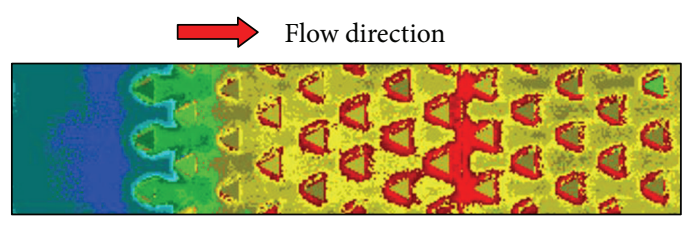

(a) Triangular pin fin array

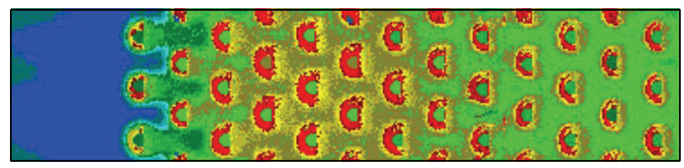

(b) Semicircular pin fin array

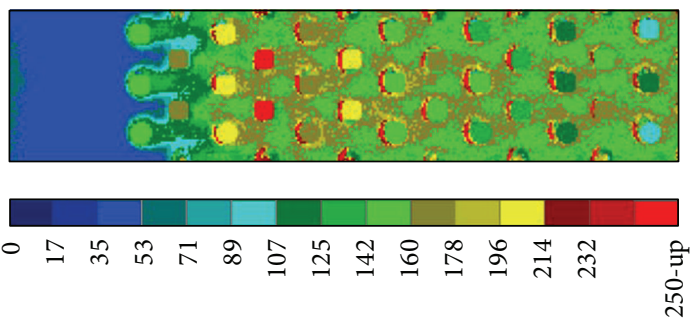

(c) Circular pin fin array

FIgURE 7: Dimensional heat transfer coefficient distributions in $\mathrm{W} / \mathrm{m}^{2} \mathrm{~K}$ units for the configurations shown in Figure 6 from Siw et al. [9].

decrease somewhat to approximate fully developed values [9]. With such fully developed conditions, local periodic values repeat themselves with streamwise development, with local periodic variations which depend upon streamwise pin fin spacing. Local values are augmented in the first few rows of both the circular and semicircular arrays because of the development and presence of horseshoe vortices around each pin fin.

As the flow develops farther downstream, local heat transfer coefficients on endwall regions around the triangular pin fins are higher than values at similar locations for the circular and semicircular arrangements. Overall, the highest overall heat transfer coefficients, which are spatially averaged over all pin fin and endwall surfaces, is produced by the triangular pin fin arrangement. According to Siw et al. [9], this is a result of the sharp edges on the triangular elements which generate wakes with increased shear, more flow mixing, and increased turbulence transport. The triangular array also gives more uniform heat transfer coefficient distributions with spatial location on the endwall [9]. 


\subsection{Dimpled Surfaces and Surfaces with Protrusion Arrays.} Recent investigations of arrays of dimples and protrusions consider flow structure from dimpled surfaces [141], Vshaped dimples [142], and effects of arrays of dimples or protrusions on heat transfer augmentation on the turn-region of a U-shaped channel used for cooling of a blade tip region [10]. Examples from this latter investigation, from Sundén et al. [10], are presented in Figures 8 and 9. The U-shaped channel arrangements, with either dimples or protrusions, are shown in Figure 8. Note that a smooth-tip two-pass channel is used as a baseline result for performance comparison. Numerically predicted dimensionless surface temperature distributions for the two arrangements are given in Figure 9, with a dimple result presented on the left and a protrusion result presented on the right. Here, inlet Reynolds number is 200,000, where Reynolds number is determined using inlet duct hydraulic diameter, and bulk spatially averaged air inlet velocity. In addition, higher numerical values of the quantity presented in Figure 9 indicate higher absolute surface temperatures. With both arrangements, local surface temperatures on the left-hand sides of each image are lower because of the impingement of cold supply fluid. Higher temperatures are then present near the side corners, farther downstream, because of local recirculating flows. In general, a larger region with lower temperatures is produced by the protrusion array arrangement, rather than by the dimpled surface arrangement. According to the investigators [10], both arrangements give increased turbulent mixing, relative to the baseline channel, with heat transfer enhancement ratios as high as 2.0. Both the dimple and protrusion arrangements are thus believed to provide effective means to increase heat transfer enhancements, with relatively small additional pressure losses.

3.3. Rib Turbulators. Recent investigations of rib turbulators address different rib configurations [143-154], as well as a variety of other geometric parameters, such as continuous and truncated ribs $[155,156]$, semiattached rib configurations [157], and effects of a turning vane arrangement within a ribbed internal cooling channel [11, 149, 158]. A variety of internal flow arrangements are addressed, including extraction flow effects and bleed holes $[159,160]$, different crossflow schemes [161], conjugate heat transfer with crossing jets [162], and impingement and effusion cooling with ribbed surfaces $[163,164]$. Other recent investigations focus on the effects of rib arrays in combination with steam cooling [165, 166] and mist/air cooling [167].

Examples from the investigation of Chen et al. [11] are given in Figures 10 and 11. Within this investigation, the effects of turning vane configuration are considered by employing the three $\mathrm{U}$-shaped internal cooling passage configurations which are shown in Figure 10: (a) test section 1 , without a turning vane in the passage turn, (b) test section 2 , with turning vane in the passage turn, and (c) test section 3 , with turning vane and ribs in the passage turn. Figure 11 presents the experimentally measured Nusselt number ratio distribution on the ribbed wall for configuration (c) for $\operatorname{Re}=30,000$, where Reynolds number is based upon inlet
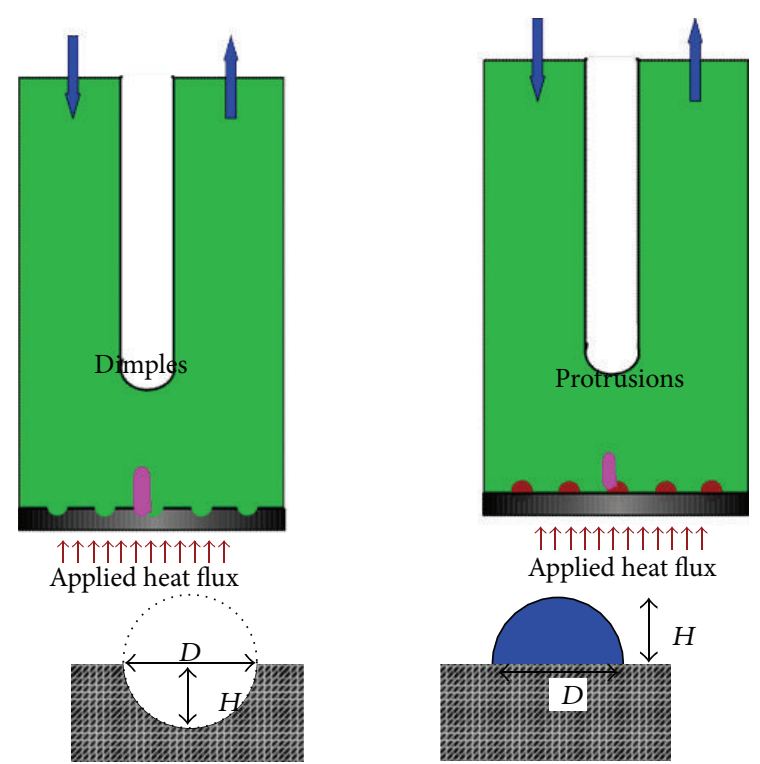

FIGURE 8: Dimple and protrusion configurations investigated in a two-pass channel for tip-wall cooling by Sundén et al. [10].

duct hydraulic diameter, and bulk spatially averaged air inlet velocity. Enhancements from the ribs in the turn region are apparent within Figure 11. However, according to Chen et al. [11], distributions for configuration (c) before and after the turn are similar to distributions for configuration (b). This leads to the conclusion that the ribs within the turn have little influence on Nusselt number ratio distributions before and after the turn. When all three configurations are considered, the overall spatially averaged Nusselt number ratio distribution is highest with configuration (a). The lowest overall pressure loss coefficient is then produced by configuration (b).

3.4. Combination Devices. Important advances in the development of internal cooling technologies for turbine components result from simultaneous use of different augmentation technologies together. A number of recent investigations consider combinations of two different types of devices $[5,6,136$, $168,169]$. For example, Amano et al. [168] investigate turbine blade cooling channels with dimples and rib-turbulators, Qui et al. [169] and Siw et al. [6] consider cooling channels with pin fins and rib turbulators, and Rao et al. $[5,136]$ employ combinations of pin fins and dimples. Use of three different devices simultaneously is discussed by Lan et al. [170] and by Murata et al. [4], who investigate internal passage cooling with combinations of rib turbulators, dimples, and protrusions. Another recent investigation focuses on internal and external cooling methods in combination, by addressing the interactions between internal ribs and external coolant film [171]. The combination and interaction of internal ribs with impingement and/or effusion cooling is described by Yang et al. [164] and Wang et al. [172]. 

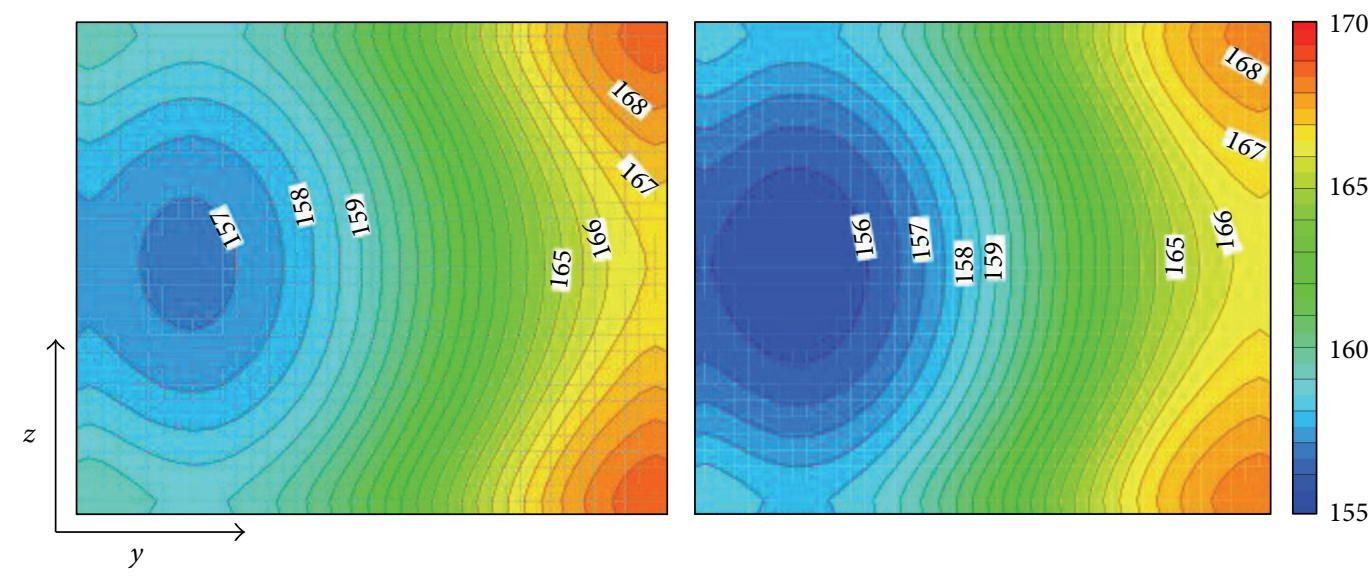

FIGURE 9: Surface temperature distributions for the dimple (left) and protrusion (right) configurations shown in Figure 8, as investigated by Sundén et al. [10].

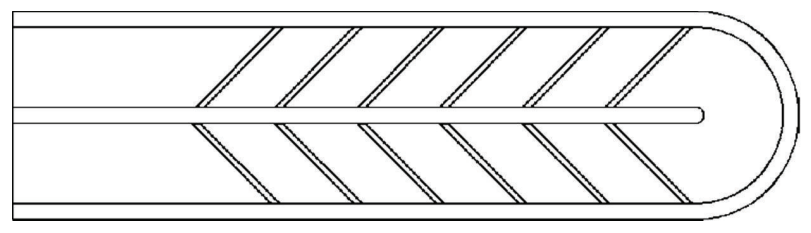

(a) Test section 1: without turning vane

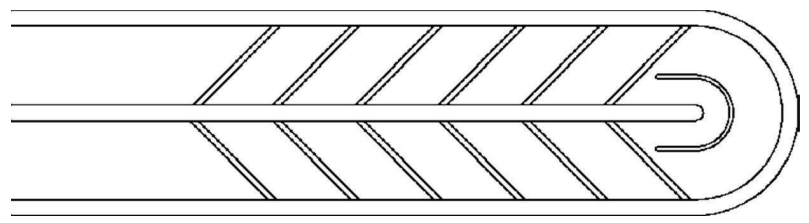

(b) Test section 2: with turning vane in turn

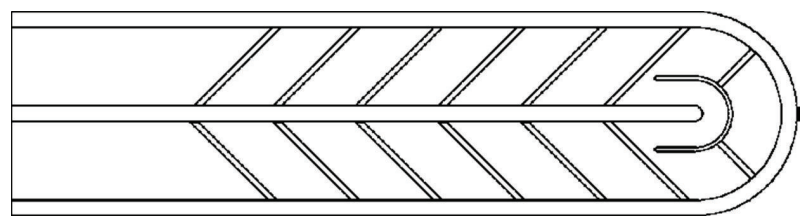

(c) Test section 3: with turning vane and ribs in turn

FIGURE 10: Vane turning test section arrangements, investigated by Chen et al. [11].

Utilizing pin fins and dimpled surfaces together is illustrated by the results which are given in Figures 12 and 13 from Rao et al. [5]. The pin fin and pin fin-dimple channel configurations which are investigated are shown in Figure 12. According to the investigators, the spacing and arrangement of the pin fin array is an optimal array arrangement for turbine airfoil cooling. The resulting endwall Nusselt number ratio distribution, presented in Figure 13, is obtained with a constant heat flux surface boundary condition for the pin fin-dimple channel with $\operatorname{Re}=18,600$, where Reynolds number is determined using inlet duct hydraulic diameter, and bulk spatially averaged air inlet velocity. Important Nusselt number ratio increases are evident near the upstream edges of the pin fins and near the downstream edges of

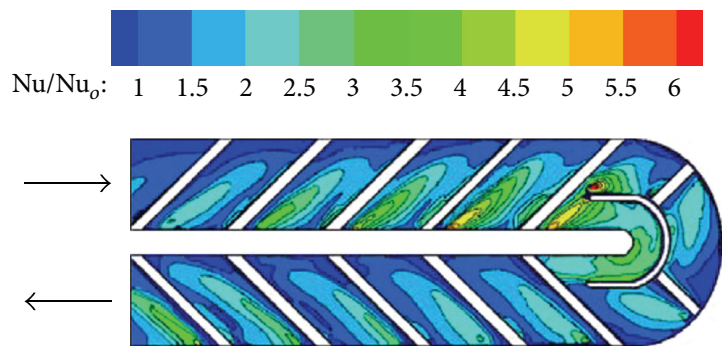

FIGURE 11: Normalized Nusselt number distribution on the ribbed wall for test section 3, experimental data for $\operatorname{Re}=30,000$, from Chen et al. [11].
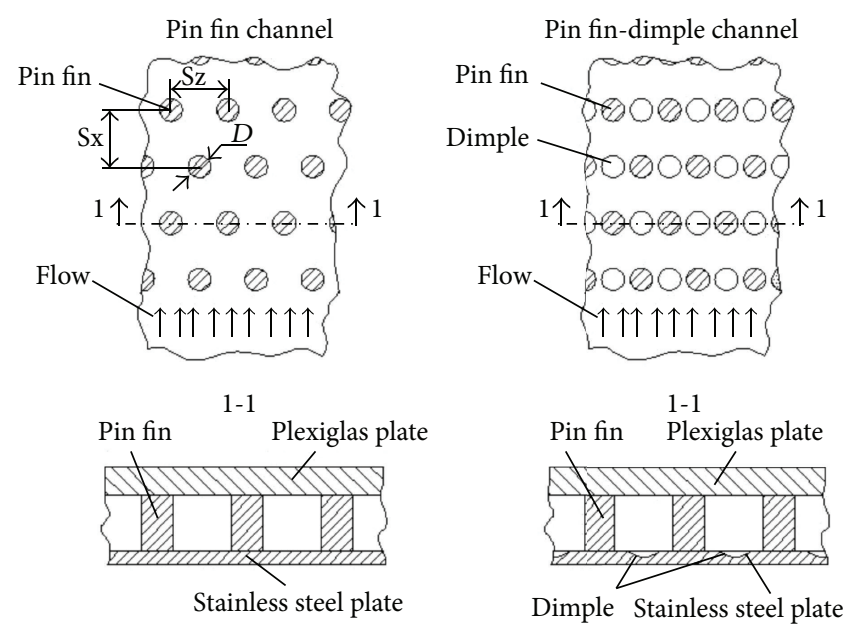

FIgURE 12: Pin fin and pin fin-dimple channel configurations considered within the channel flow investigation by Rao et al. [5].

the dimples. The former variations are mostly due to the horseshoe vortices which form at the pin fin/endwall junction $[1,8]$, whereas the latter are a result of the different vortex structures which are periodically shed from the individual dimples $[1,48,52]$. The results from this investigation show that overall endwall heat transfer enhancements are larger for the pin fin-dimple channel, relative to the channel with 


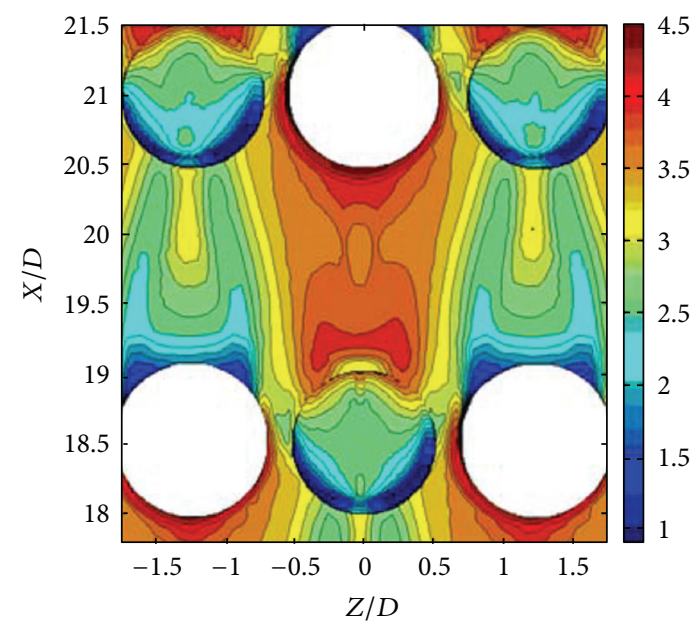

FIGURE 13: Local Nusselt number ratios on the endwall of the pin fin-dimple channel for $\mathrm{Re}=18,600$ from the investigation by Rao et al. [5].

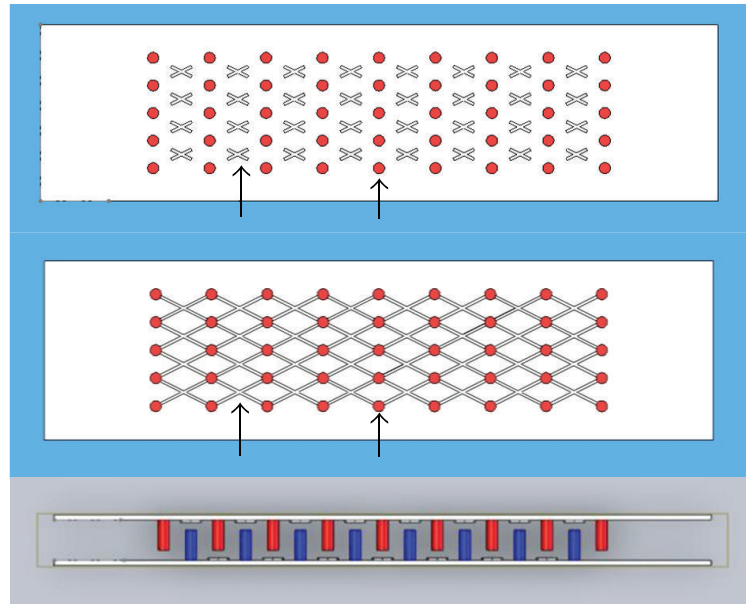

FIGURE 14: Broken rib, full rib array, and side view of staggered pin fin configuration from Siw et al. [6].

pin fins only. The investigators [5] attribute this to increased turbulent mixing near the wall caused by strong vortical flow shedding from the dimples.

Examples which illustrate the combined use of pin fins and ribs are presented in Figures 14 and 15, from Siw et al. [6]. The broken rib array, full rib array, and side view of the staggered pin fin configuration, which are utilized, are shown in Figure 14. The resulting dimensional heat transfer coefficient distributions for a Reynolds number of 25,000 are then given in Figure 15 (where Reynolds number is determined using inlet duct hydraulic diameter, and bulk spatially averaged air inlet velocity). Here, the ratio of detached spacing between the pin-tip and the endwall, and pin diameter is 2 . The ratio of pin height and pin diameter is also 2. Inspection of Figure 15 reveals that the largest heat transfer coefficient values are generally present with the broken rib arrangement, most especially at many endwall locations. The investigators [6] attribute this behavior to excessive vortices which are

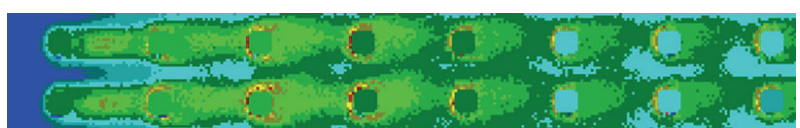

(a) Baseline-no ribs

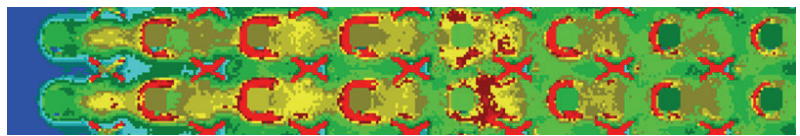

(b) Broken ribs

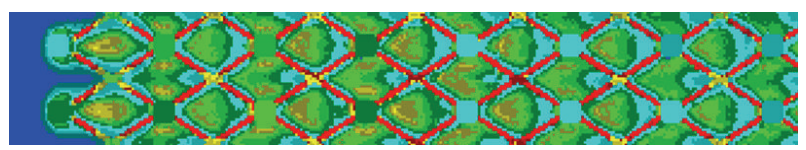

$\begin{array}{llllllllllllllll}0 & 21 & 42 & 64 & 85 & 107 & 128 & 150 & 171 & 192 & 214 & 235 & 257 & 278 & 300 & 320\end{array}$

(c) Full ribs

FIGURE 15: Local dimensional heat transfer coefficient distributions $\left(\mathrm{W} / \mathrm{m}^{2} \mathrm{~K}\right)$ for configurations shown in Figure 14 for $\mathrm{Re}=25,000$ from Siw et al. [6].

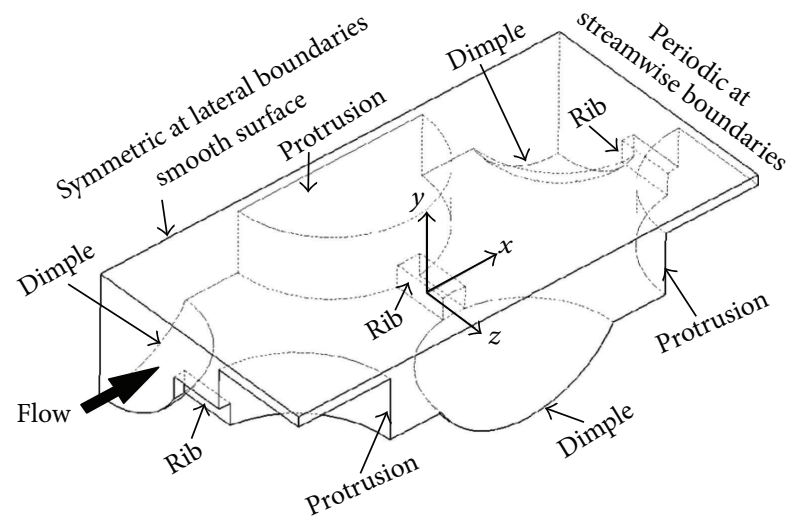

FIGURE 16: Rib, dimple, protrusion arrangement, illustrating domain for numerical analysis, employed in the investigation by Murata et al. [4].

generated by the broken ribs. The development of these vortices seems to occur as horseshoe vortices, which are also a major contributor to heat transfer enhancement, additionally continue to be present.

The combined use of rib turbulators, dimples, and protrusions is illustrated by the results which are given in Figures 16 and 17 [4]. The experimental Nusselt number ratio data in the latter figure are presented for the overall arrangement shown in Figure 16 for a Reynolds number of 2,000, with two different protrusion sizes. Here, Reynolds number is based upon bulk spatially averaged air inlet velocity, and a length scale equal to two times channel height. Even though the Reynolds number for this investigation is relatively low, the results presented are representative of fully turbulent flow conditions. Note that the data presented in Figure 17 are given for ratios of protrusion diameter to channel height of 2.54 and 1.70. According to Murata et al. [4], Figure 17 shows that the highest heat transfer augmentations are present near 

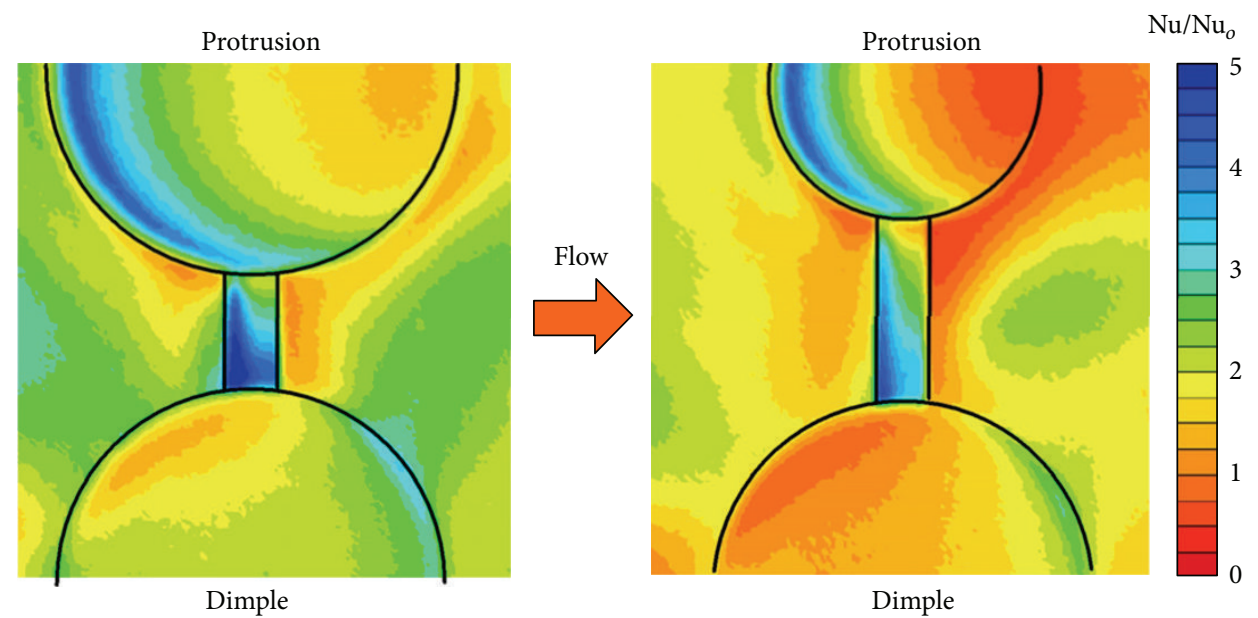

FIGURE 17: Local Nusselt number ratios for the rib, dimple, and protrusion arrangement employed in the investigation by Murata et al. [4] for $\mathrm{Re}=2,000$ and two different protrusion sizes.

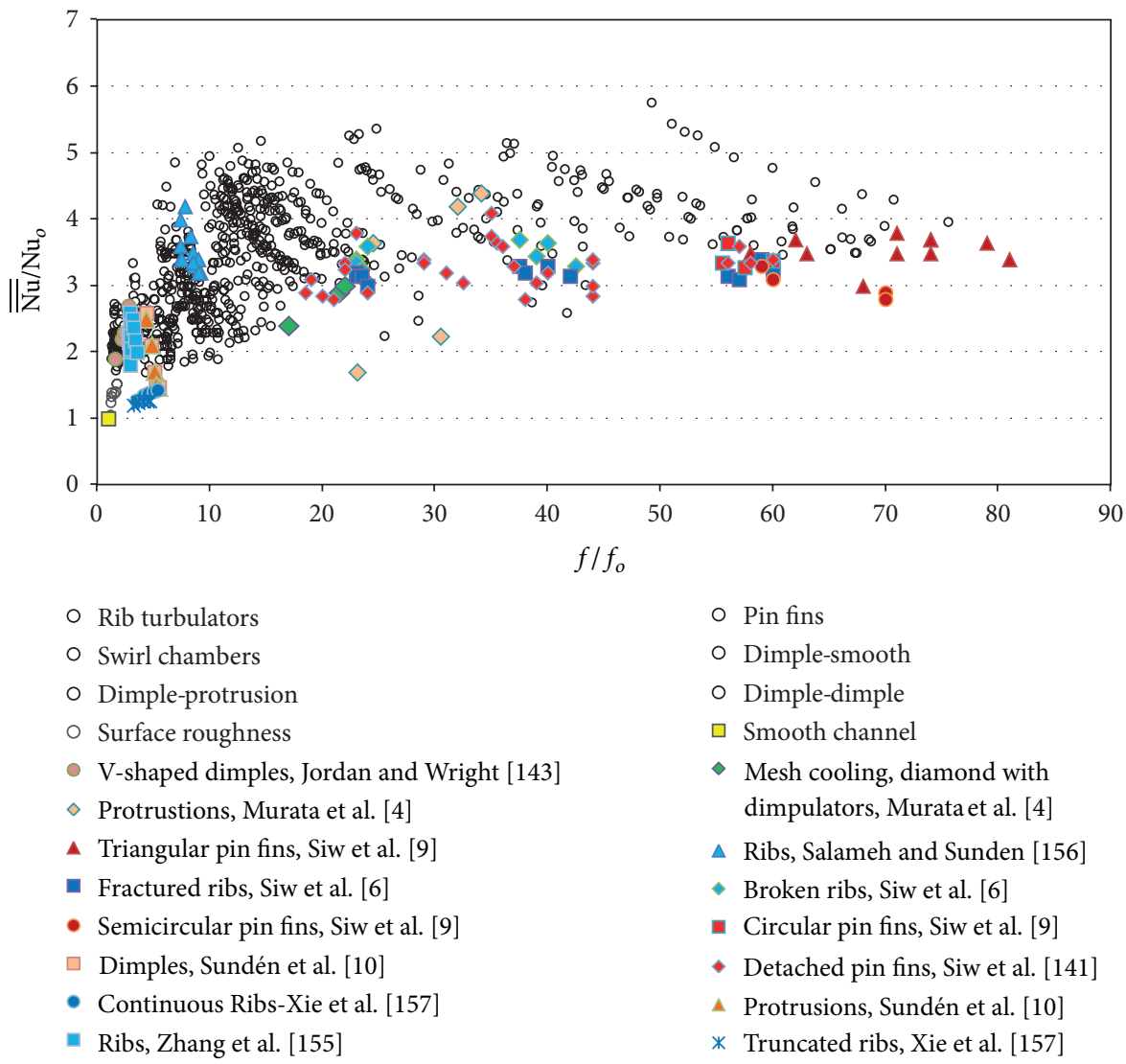

FIGURE 18: Comparison of relative performance of rib turbulators, pin fins, swirl chambers, dimple-smooth arrangements, dimple-protrusion arrangements, dimple-dimple arrangements, surface roughness, and smooth-walled channels, including results from investigations after 2003. Globally averaged Nusselt number ratios $\overline{\overline{\mathrm{Nu}}} / \mathrm{Nu}_{o}$ are presented as dependent upon friction factor ratios $f / f_{o}$.

the leading edge of the protrusion and on the top of the rib surface. Important augmentations are also occurring near the downstream edges of the dimples, as well as on an angled trajectory between adjacent dimples, which is a consequence of the flow blockage produced by the protrusions. When the effects of protrusion diameter are considered, Figure 17 also shows that Nusselt number ratios are higher on the protrusion and ribs when the protrusion diameter is larger. With the smaller protrusion diameter, an isolated high Nusselt number ratio region is observed downstream of the 


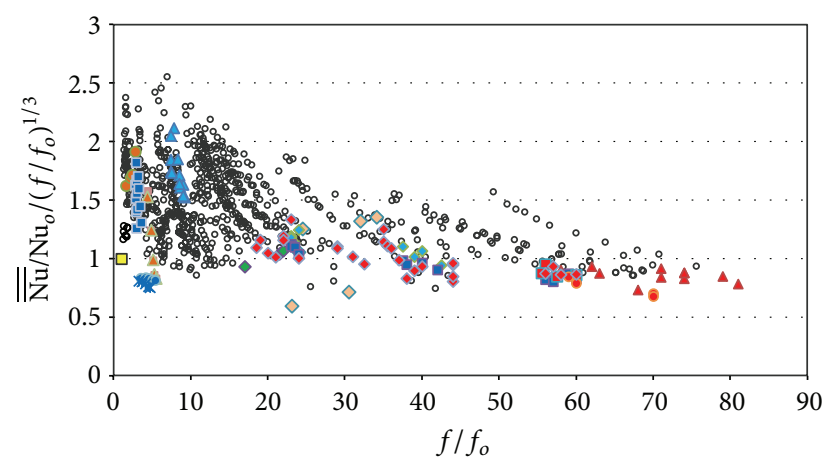

FIGURE 19: Comparison of relative performance of rib turbulators, pin fins, swirl chambers, dimple-smooth arrangements, dimpleprotrusion arrangements, dimple-dimple arrangements, surface roughness, and smooth-walled channels, including results from investigations after 2003. Globally averaged thermal performance parameters $\overline{\overline{\mathrm{Nu}}} / \mathrm{Nu}_{o} /\left(f / f_{o}\right)^{1 / 3}$ are presented as dependent upon friction factor ratios $f / f_{o}$. Symbol definitions are given in Figure 18.

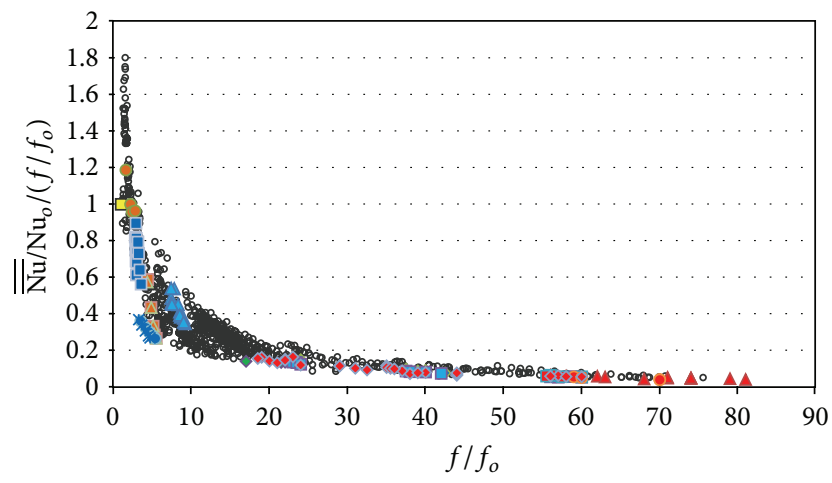

Figure 20: Comparison of relative performance of rib turbulators, pin fins, swirl chambers, dimple-smooth arrangements, dimpleprotrusion arrangements, dimple-dimple arrangements, surface roughness, and smooth-walled channels, including results from investigations after 2003. Globally averaged thermal performance parameters $\overline{\overline{\mathrm{Nu}}} / \mathrm{Nu}_{o} /\left(f / f_{o}\right)$ are presented as dependent upon friction factor ratios $f / f_{o}$. Symbol definitions are given in Figure 18.

transverse rib segment, which is due to reattachment of the shear layer, which forms above the flow separation region present just downstream of the rib.

The combined use of inclined pin fins and wavy endwalls is described by Takeishi et al. [174] and Motoda et al. [175]. Typically, pin fins with angles of plus and minus 45 degrees, relative to the flat endwall plane, are employed. Overall, spatially averaged heat transfer rates are higher on endwalls with normal pin fins, compared to endwalls with angled pin fins. However, overall heat transfer increases (for the entire passage surface area) are present for the angled pin fin arrangement because of an increase of overall surface area by a factor of 1.4. Associated pressure drop penalties are then generally lower when inclined pin fins are employed. With wavy endwalls also utilized, the average heat transfer coefficient of the -45 degree inclined pin fins is about the

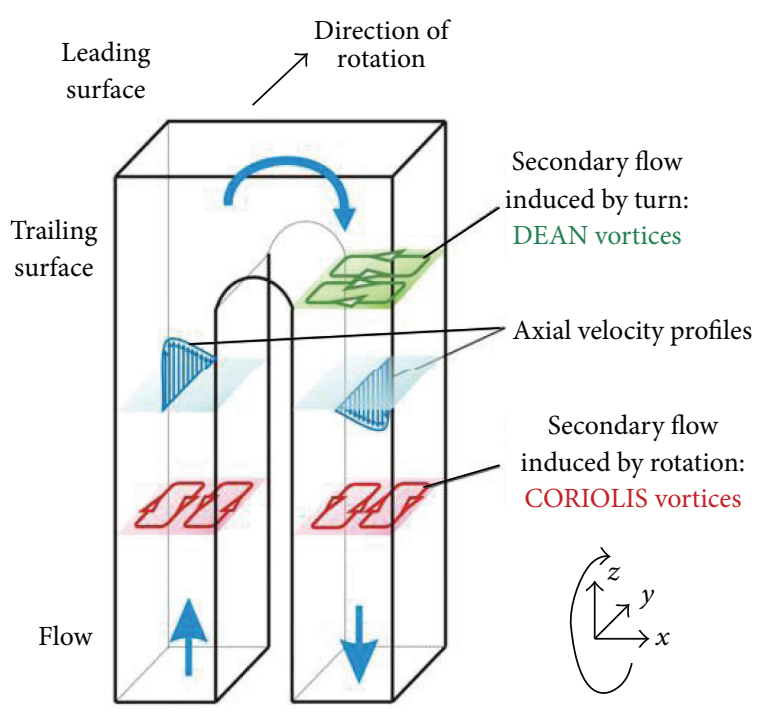

FIGURE 21: Schematic diagram illustrating typical channel arrangement and orientation relative to rotational motion, including resulting secondary flows [50].

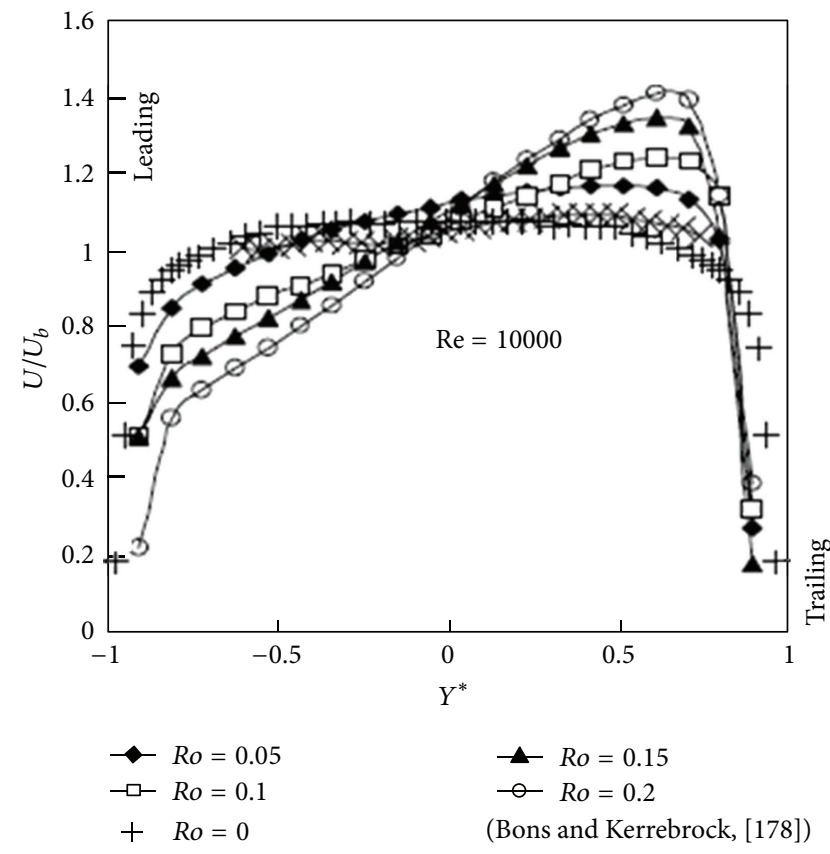

FIGURE 22: Midspan velocity profile for outward radial flow in a rotating smooth-walled channel from Chen and Liou [101].

same or a little higher than the heat transfer coefficients of pin fins mounted on a flat endwall. However, the overall pressure loss penalty of the -45 degree inclined pin fins with a wavy endwall is generally less than the penalty when a flat endwall is used.

3.5. Overall Comparisons. Figure 18 presents comparisons of globally averaged Nusselt number ratios $\overline{\overline{\mathrm{Nu}}} / \mathrm{Nu}_{o}$ and friction 


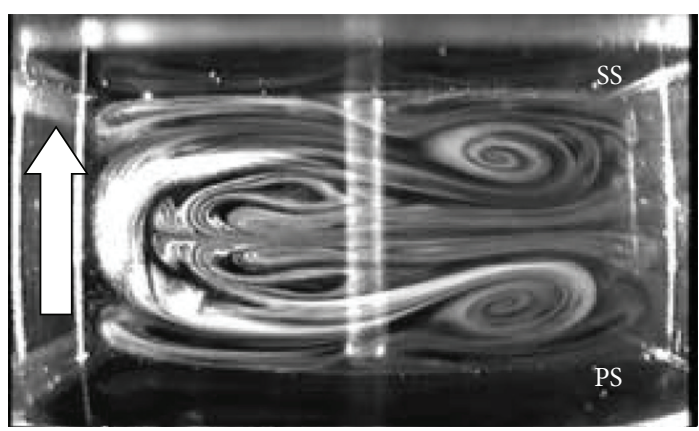

(a)

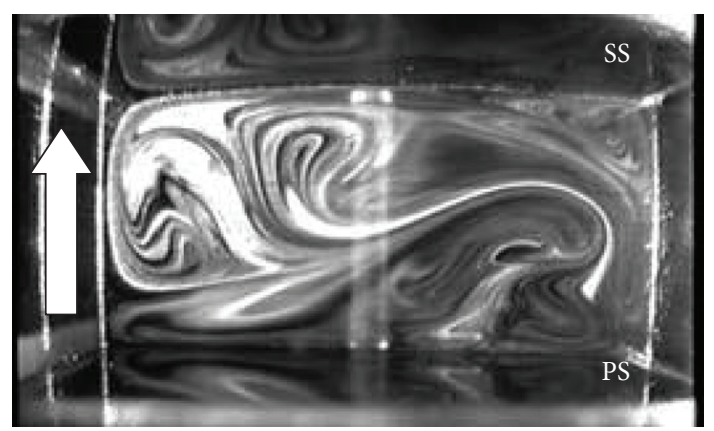

(b)

FIGURE 23: Flow visualization images from Elfert et al. [50] for the $180^{\circ}$ bend region of the two-pass channel, viewing the flow in the negative $z$-direction with the bend entrance on the left and the bend exit on the right. (a) Image without rotation. (b) Image with rotation.

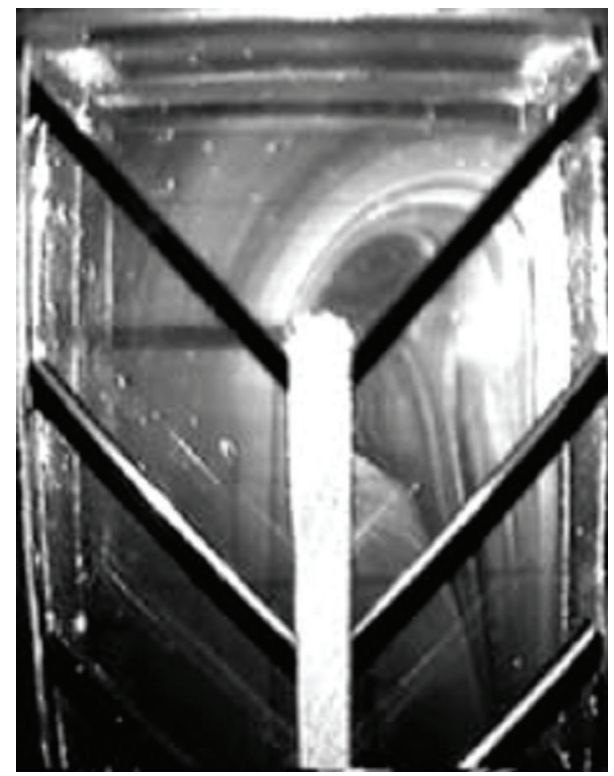

Figure 24: Flow visualization image from Elfert et al. [50] for the $180^{\circ}$ bend region of the two-pass channel, viewing the flow in the positive $y$-direction with the bend entrance on the left and the bend exit on the right.

factor ratios $f / f_{o}$ for rib turbulators, pin fins, swirl chambers, different dimple and protrusion arrangements, surface roughness, and smooth-walled channels. Included within Figure 18 are results from investigations since 2003. In regard to these more recent studies, data from pin fin investigations $[9,140]$ are designated with red symbols, data from rib investigations $[6,154-156]$ are designated with blue symbols, data from one mesh cooling study [176] are designated with green symbols, and data from investigations which employ dimples and protrusions $[4,10,142]$ are designated with brown symbols. Note that some of the combination device results from the previous section $[5,11,136,164,168-172]$ are not included since friction factor ratio data are not available in these studies. The mesh cooling arrangement, employed by Bunker et al. [176], employs a "small diamond with dimpulators" arrangement. Here, the ratio of dimple print diameter to channel height is 3.0, ratio of protrusion diameter to channel height is 3.5, and ratio of rib height to channel height is 0.15 . Description of this mesh cooling arrangement is also provided by Murata et al. [4]. Also included in Figure 18 are a Nusselt number ratio and a friction factor ratio for a smooth channel (designated with a yellow symbol), to provide a baseline comparison. Inspection of Figure 18 reveals that all of the new data, obtained since 2003, collect within scatter of the data obtained from investigations conducted prior to 2003 [1]. The arrangement with the most promising performance characteristics may be the rib investigation of Salameh and Sunden [155], since Nusselt number ratios are as high as 4.2 with a corresponding friction factor ratio of 7.8. The ribs in this investigation are placed transversely to the direction of the main flow on the outer wall of the bend of a $\mathrm{U}$-shaped duct. The ratio of rib height to hydraulic diameter is 0.1 , and the rib pitch ratio is 10 . Both continuous and truncated ribs are considered.

The data in Figure 18 are also presented again in Figures 19 and 20. In the first of these figures, globally averaged thermal performance parameters $\overline{\overline{\mathrm{Nu}}} / \mathrm{Nu}_{o} /\left(f / f_{o}\right)^{1 / 3}$ are given as dependent upon the friction factor ratio $f / f_{o}$. The $\overline{\overline{\mathrm{Nu}}} / \mathrm{Nu}_{o} /\left(f / f_{o}\right)$ parameter, presented in Figure 20, is referred to as the Reynolds analogy performance parameter. Like the results in the previous figure, Figures 19 and 20 show that all of the new data, obtained since 2003, are collected within distributions of the data obtained from investigations conducted prior to 2003 [1].

\section{Effects of Rotation}

Fluid flows within rotating channels are different from those in stationary channels because of the development of secondary flows, such as Coriolis vortices, from rotational influences. As a result, local surface heat transfer and friction factor augmentation levels are often altered because of the presence of rotation. 
Figure 21 shows a typical turbine blade cooling passage arrangement from Elfert et al. [50]. Here, a two-pass system is employed, which is rotating in an orthogonal mode, such that the flat surface which comprises the $180^{\circ}$ bend between the two passes is parallel to the axis of rotation. Note that the rotation is directed from the trailing side to the leading side of the passage. In modeling a turbine blade, the leading and trailing surfaces are analogous to the suction and pressure sides, respectively.

According to Servouze and Sturgis [177], the Coriolis force is an apparent force that exists in the noninertial, rotating reference frame. Application of the Coriolis effect on radially outward flow results in a force acting towards the pressure side, whereas application of the Coriolis effect on radially inward flow results in a force acting towards the suction side. As a result, Coriolis forces often induce secondary flows in fluid flows contained within rotating systems. Counterrotating vortex pairs then develop in both radially outward flows and radially inward flows, with different directions of vortex rotation for different flow directions (relative to pressure and suction sides of the passage). Such vortex pairs are illustrated in Figure 21. Experimental evidence of Coriolis vortex pairs in rotating channels is provided by Elfert et al. [50] and Bons and Kerrebrock [178] from Particle Image Velocimetry (PIV) experiments, as well as by Chen and Liou [101] from Laser-Doppler Velocimetry (LDV) experiments.

The axial velocity profiles, also shown in Figure 21, are altered because of the presence of Coriolis vortices. Midspan velocity profiles for radially outward flow in a rotating smooth-walled channel from Chen and Liou [101] are presented in Figure 22. From these results, it is evident that, as rotation number increases, velocity increases near the pressure (or trailing) side and decreases near the suction (or leading) side relative to the mean stream velocity. Other experimental results from Chen and Liou [39] show that the opposite velocity profile trend is present for radially inward flow. Here, overall deviations and skewness (relative to the mean stream velocity) are quantitatively less as rotation number increases (relative to radially outward flow).

Figure 21 also shows Dean vortices near the exit of the $180^{\circ}$ turn and near the beginning of the segment of radially inward channel flow. Dean vortices develop as a result of curvature of flow streamlines around the passage bend. A visualization of such a Dean vortex pair is shown in Figure 23(a) from Elfert et al. [50] for a stationary, nonrotating channel. With this arrangement, the vortex pair is approximately symmetric. With rotation present, the image in Figure 23(b) is obtained with Coriolis vortices on the left side of the image, before the flow enters the turn, and distorted Dean vortices on the right side, just before the flow exits the turn. Here, the influences of rotation cause the vortices near the suction side to become more coherent, whereas the vortices near the pressure side are more distorted and diffuse. This trend continues until local mixing within and around the pressure side vortices dominates the channel, as illustrated by the image in Figure 23(b). Overall, diffuse and convoluted secondary flows and the associated thermal transport mechanisms are most important near the pressure surface.
Another important event associated with turning ducts is formation of a separation flow region on the inner wall of the channel immediately following the bend, as shown in Figure 24. According to Elfert et al. [50], the characteristics and extent of such a separation region depend upon flow characteristics, such as flow speed, channel geometry, and channel surface condition.

4.1. Effects of Rotation on Local Nusselt Numbers, Spatially Averaged Nusselt Numbers, and Overall Thermal Performance. In general, rotation appears to alter heat transfer distributions only over very limited portions of channel surfaces. Existing investigations provide little evidence of overall increases or decreases in overall thermal performance characteristics with rotation, at any value of rotation number, buoyancy parameter, density ratio, or Reynolds number. In many investigations of internal passage flows with heat transfer and rotation, the Reynolds number and rotation number are given by

$$
\begin{aligned}
& \mathrm{Re}=\frac{U D_{h}}{v}, \\
& \mathrm{Ro}=\frac{\Omega D_{h}}{U},
\end{aligned}
$$

respectively. The inlet density ratio is then expressed using an equation of the form

$$
D R=T_{c}\left(\frac{1}{T_{w}}-\frac{1}{T_{c}}\right)=\frac{\left(\rho_{w}-\rho_{c}\right)}{\rho_{c}} .
$$

The buoyancy parameter is then given by the following,

$$
B o=\operatorname{Ro}^{2}\left(\frac{R}{D_{h}}\right) D R .
$$

The relationships and interdependence of these different parameters are apparent from these definitions.

In regard to overall rotation effects, the investigations of Fu et al. [128], Wright et al. [179], and Acharya et al. [180] generally show that overall thermal performance in smooth channels is affected more by Reynolds number than rotation number. According to Fu et al. [128], pressure losses and Reynolds number are generally insensitive to rotation number. A similar conclusion is reached if ribbed turbulators are present in rotating channels [102, 173, 179, 181, 182].

This is largely because of the competing effects of rotation on the pressure (or trailing) sides and the suction (or leading) sides of internal channel flows. According to Huh et al. [104], differences in local Nusselt number ratios for pressure sides and suction sides are generally a result of the rotation induced secondary flows caused by Coriolis vortices. These investigators indicate that (for radially outward flow channels) the effects of these secondary flows are generally more pronounced on the pressure sides of channels, where larger local heat transfer augmentations are often present (compared to suction sides).

This qualitative trend is often reversed for channels with multiple passes and turns $[58,64,65,74,75,77,81,100$, 
$104,128,177,182-206]$. This is because, after the first turn, flow changes from radially outward to radially inward, which alters the application direction of the Coriolis force. The overall result for radially inward flow is generally surface heat transfer augmentations on passage suction sides. However, note that for channels with more than two passes, the quantitative consequences of the change in flow direction often become less pronounced in later passages [74, 185, 188, 189, 194, 202-204, 207].

A number of recent investigations show that rotation effects on Nusselt number ratios are often suppressed at high Reynolds numbers. This is illustrated by Nusselt number ratios which tend toward stationary channel values as Reynolds number increases [58, 64, 65, 74, 82, 104, 128, 182$184,186,187,191,200,201,206,208-214]$. One rationale for this behavior is the inverse dependence of Reynolds number and rotation number, which means that rotation number generally decreases as the associated Reynolds number increases (provided certain experimental parameters are maintained constant). Investigations which maintain constant Reynolds number show that Nusselt number ratios deviate further from stationary values with increasing rotation number $[103,189$, 193, 202, 203, 215-218].

According to Wright et al. [219], rotation effects sometimes become more pronounced at lower Reynolds numbers when rib turbulators are employed. In general, according to this study, for Reynolds numbers from 10,000 to 40,000, the higher thermal performance characteristics, with no rotation, do not change significantly when rotation is added. The only exception is observed for $\operatorname{Re}=10,000$, wherein some improvement in overall thermal performance with rotation number is present [219].

For radially outward flow channels, Nusselt number ratio tends to decrease asymptotically with streamwise development from the channel entrance, with pressure side Nusselt number ratios higher than suction side values $[64,74$, $75,103,104,128,178,179,183,185-193,196-198,200,201$, 204-211, 215, 220-230]. Such trends are due to developing thermal boundary layers. When rib turbulators are present, the asymptotic decrease is less pronounced; however, Nusselt number ratios continue to be larger on the pressure side than on the suction side $[58,64-66,75,77,81,82,100,128,177,179$, $182-184,186,189,194,199,208,209,212,213,220,229,231-$ 235].

Many investigations show significant increases in Nusselt number ratio as internal passages turn from radially outward to radially inward flow, and from radially inward flow to radially outward flow $[64,65,74,75,77,104,128,173,182$, 183, 185, 187-189, 191-194, 196-201, 204, 206, 207, 214, 215, $232,236]$. This local augmentation is generally attributed to the flow impinging on the outer walls of the turn, as well as the secondary flows resulting from the presence of Dean vortices. Other investigations show slight decreases in Nusselt number ratio in turn regions, especially when rib turbulators are employed [58, 74, 183, 184, 186, 189, 202, 203]. Such variations may be due to shear, separation, and reattachment phenomena which become more important than Dean vortex effects at high Reynolds numbers $[64,65,74,104,128,182-$ $184,186]$.
A number of investigations consider rotating channels with a variable wall temperature surface boundary condition $[128,186,194,201,207,215,217,218,227,228,234,236,237]$. In many of these investigations, wall temperature increases along the length of the channel, with slight decreases through turn regions.

Investigations which employ naphthalene sublimation to determine mass transfer generally obtain results which are consistent with heat transfer experiments [216, 238-247]. However, one drawback of such mass transfer methods is the absence of buoyancy within experiments.

Several other experimental investigations of heat transfer and flow behavior in rotating channels consider effects of channel shape and orientation [169, 248-251], pin fins [169, 251, 252], impingement cooling [253], dimpled surfaces [254], arrays of spherical protrusions [255], trailing edge cooling [256-258], and rib turbulators [148, 149, 166, 169, 259, 260]. For example, Tse and Steubner [98] measure different velocity components in a serpentine channel with $45^{\circ}$ ribs. Prabhu and Vedula [83] measure surface static pressure variations with different channel aspect ratios, and different rotational speeds in a rectangular channel with tranverse ribs on one wall. Compared with results from nonrotating ribbed channels $[59,87]$, important effects of rotation are evident in the results from both investigations.

Computational studies of flows and heat transfer in ducts with rib turbulators consider straight single-pass ducts [56, $72,73,81,86,89,99]$, two-pass ducts [57], two-pass ducts with U-shaped channels in between $[60,76,84,88,90]$, $90^{\circ}$ orthogonal ribs $[72,73,81,84,86,99], 45^{\circ}$ angled ribs $[56,57,60,76,88-90], 45^{\circ} \mathrm{V}$-shaped ribs [73], and rotation $[56,57,60,72,76,81,84,88,90]$. In one case [89], the angled ribs placed on two opposite walls of the channel are rounded. The results from the most notable of these investigations with rotation show that the secondary flows induced by angled ribs, rotating buoyancy, and Coriolis forces produce alterations to surface heat transfer coefficients, and flow field structure. This includes the development of strong nonisotropic turbulence stresses [88, 90]. Jia et al. [73] and Watanabe and Takahashi [99] include experimentally measured friction factors, Nusselt numbers, and streamwise velocity fluctuations, which are compared to numerically predicted results. Experimental investigation of a complex trailing edge passage of a high pressure turbine blade is described by Bunker et al. [61]. Rigby and Bunker [85] employ a full three-dimensional Navier-Stokes equation solver to investigate the same arrangement.

4.2. Effects of Rotation Number. Figure 25 from Zhou and Acharya [102] shows how spatially averaged pressure and suction side Nusselt number ratios vary with rotation number. Of course, different trends with rotation number are present for the two sides of the rotating channel with radially outward flow. These experimental results from Zhou and Acharya [102] are obtained for both portions of a two-pass channel. As a result, opposite trends with rotation number are also evident in Figure 25 for the suction and pressure 

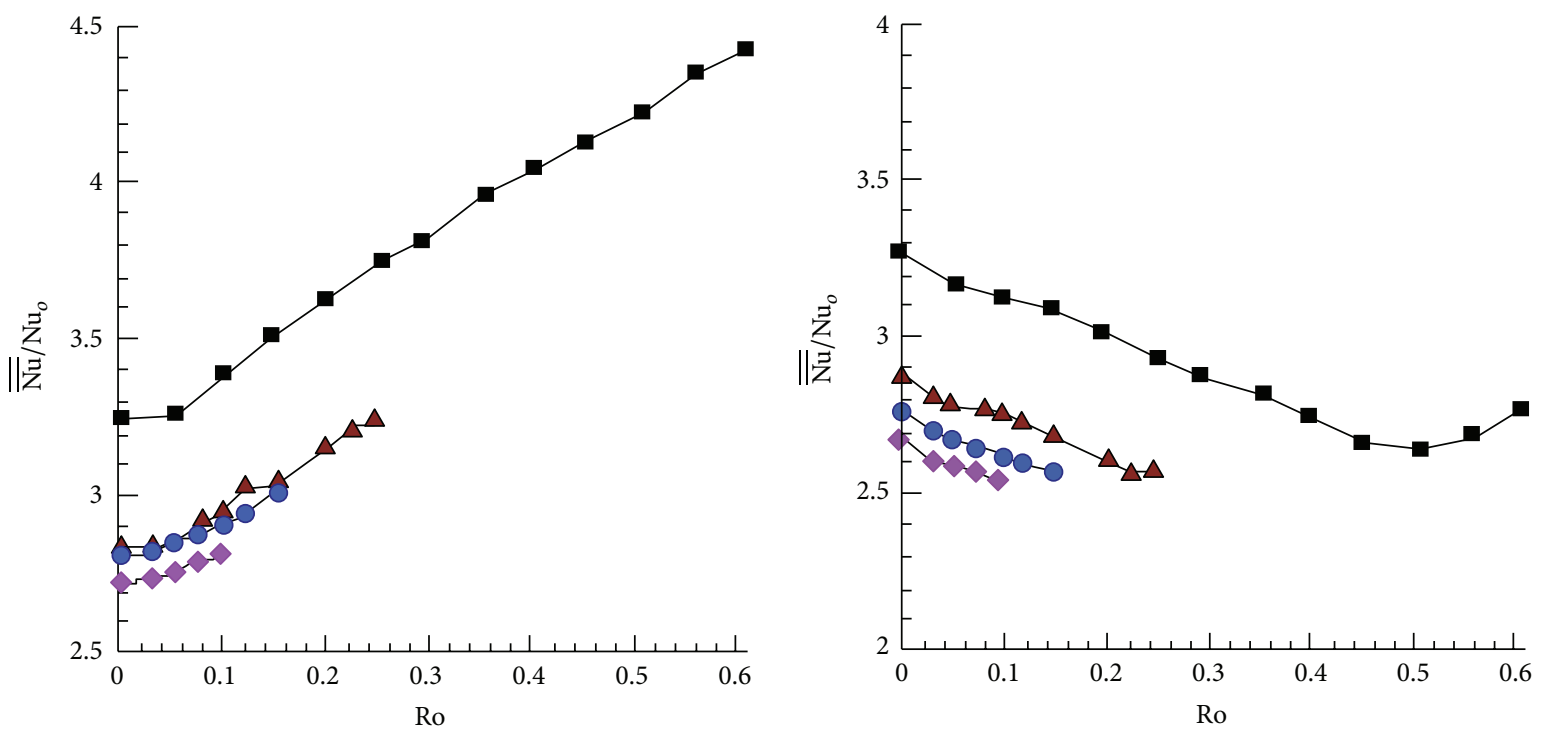

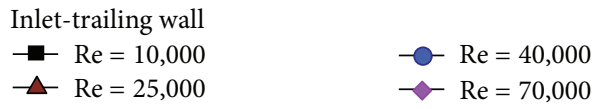

(a)

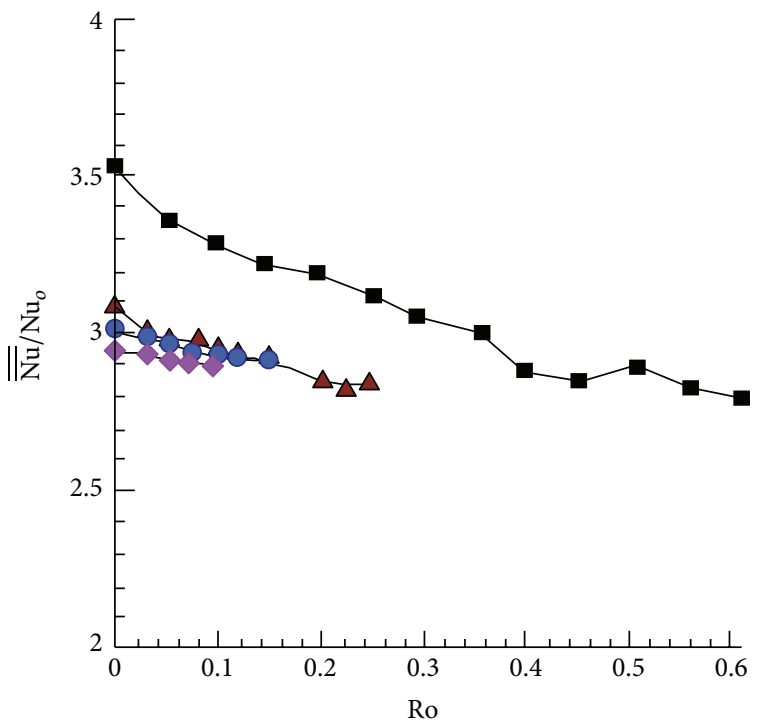

Outlet-trailing wall

- $\operatorname{Re}=10,000$

$\triangle \mathrm{Re}=25,000$

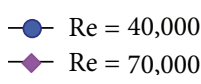

(c)
Inlet-leading wall

$\rightarrow \operatorname{Re}=10,000$

$\triangle \operatorname{Re}=25,000$

$\operatorname{Re}=40,000$

(b)

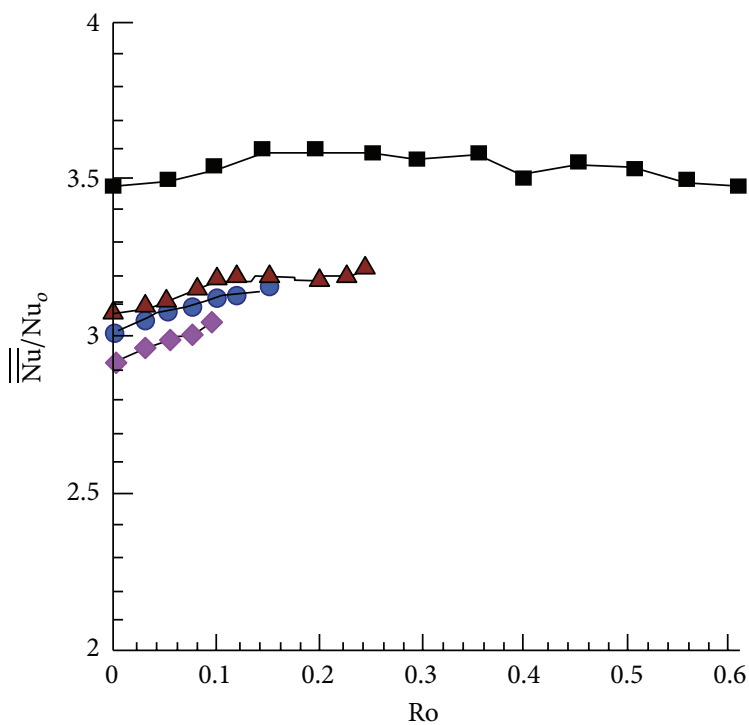

Outlet-leading wall

$-\operatorname{Re}=10,000$
$\triangle \operatorname{Re}=25,000$

$\mathrm{Re}=40,000$

$\mathrm{Re}=70,000$

(d)

FIGURE 25: Spatially averaged Nusselt number ratio variations as dependent upon rotation number for the rotating channel investigation of Zhou and Acharya [102]. (a) Radial outflow pressure side results. (b) Radial outflow suction side results. (c) Radial inflow pressure side results. (d) Radial inflow suction side results.

sides for radially inward flow $[58,64,65,74,75,82,102-$ $104,179,180,184,185,188-191,193,194,196,200,202-212,216-$ 218, 221-223, 230, 237, 239, 241, 261-264].

Overall, the results in Figure 25 show that Nusselt number ratios increase with rotation number for the pressure side with radial outward flow, and decrease with rotation number for the suction side with radial outward flow. Decreases with rotation number are then present for the pressure side with radial inward flow, and increases are present with rotation number for the suction side with radial inward flow. Because of such competing effects on each passage portion, overall, spatially averaged Nusselt numbers are generally not significantly different from values, measured in similar configurations, with no rotation. For many turbine thermal 

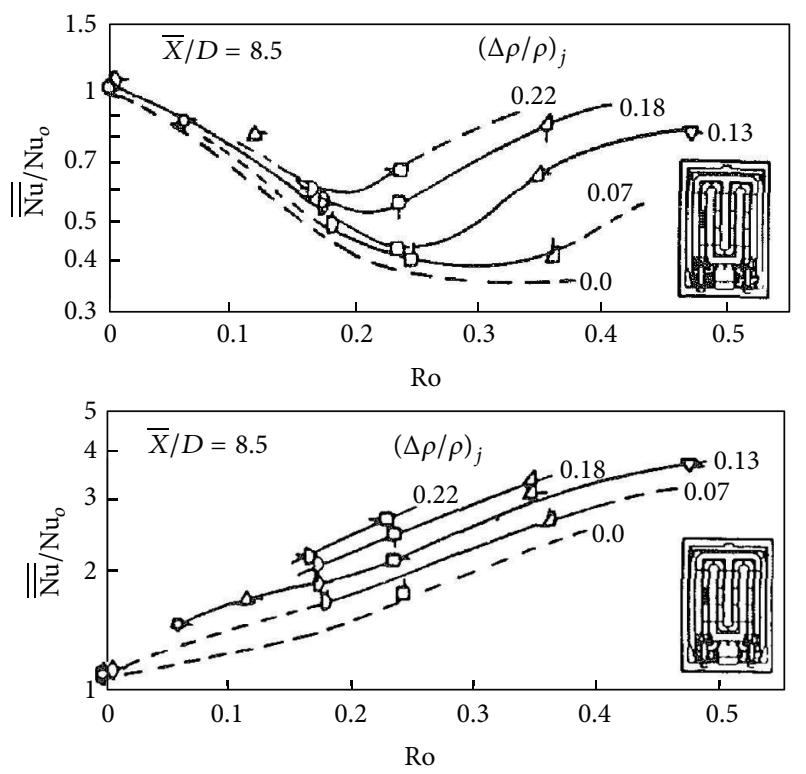

Figure 26: Spatially averaged Nusselt number ratio variations as dependent upon inlet density ratio and rotation number for the rotating channel investigation of Wagner et al. [103]. Results for a radial outflow arrangement are presented for suction (top figure) and pressure (bottom figure) sides of the rotating passage.

designers, local Nusselt number reductions are as, or more important, than local augmentations. This is because such degradations of local heat transfer coefficients also must be understood and correctly accommodated within designs in order to insure appropriate engine longevity.

4.3. Effects of Inlet Density Ratio. Figure 26 presents spatially averaged Nusselt number ratio variations as dependent upon inlet density ratio and rotation number for the rotating channel investigation of Wagner et al. [103]. Results for a radial outflow arrangement are presented for suction (top figure) and pressure (bottom figure) sides of the rotating passage. Overall, these data show that increasing the inlet density ratio enhances the Nusselt number ratio for both pressure and suction side surfaces $[74,102,103,173,188$, 189, 202-204, 206, 235, 265, 266]. From Figure 26, it is apparent that the density ratio has a significant influence on heat transfer magnitudes over a range of rotation numbers. According to Hajek et al. [189], inlet density ratio has a slightly larger effect on the pressure side than the suction side and diminishing influences in later passages of multipass and serpentine channels. Because the buoyancy parameter is proportional to the product of the inlet density ratio and rotation number squared, alteration of these different parameters results in different data trends.

4.4. Effects of Buoyancy Parameter. Figure 27 from Huh et al. [104] shows that local area-averaged Nusselt number ratios are affected by variations of the buoyancy parameter in the same approximate qualitative manner as when the rotation number changes. Note that the buoyancy parameter

\begin{tabular}{|c|c|c|c|c|c|}
\hline Re & $10 \mathrm{~K}$ & $15 \mathrm{~K}$ & $20 \mathrm{~K}$ & $30 \mathrm{~K}$ & $40 \mathrm{~K}$ \\
\hline Trailing & $\triangle$ & $\square$ & $\searrow$ & $\bigcirc$ & + \\
\hline Leading & $\triangle$ & $\square$ & $\searrow$ & $\bigcirc$ & + \\
\hline
\end{tabular}
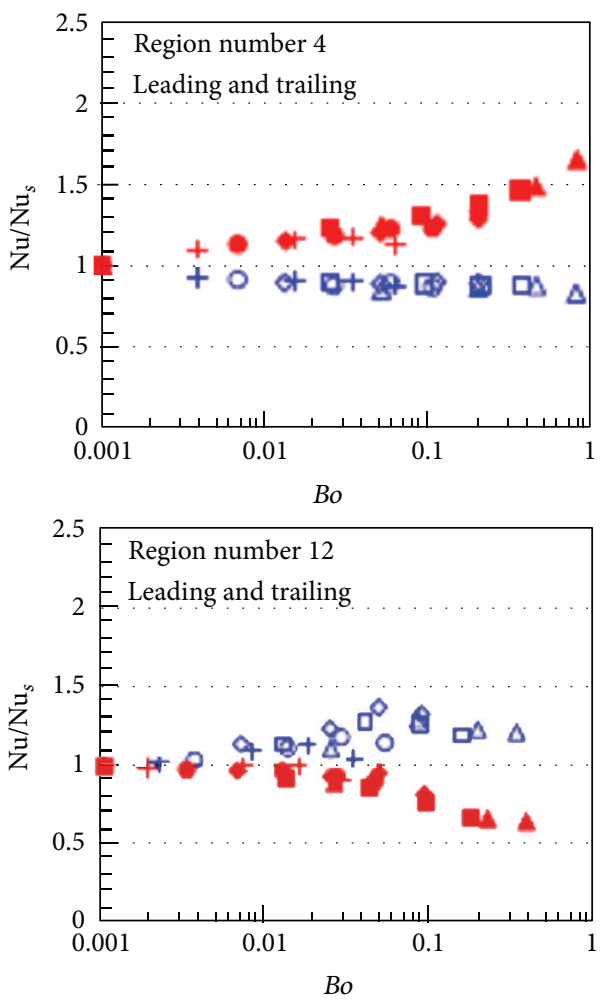

FIgURE 27: Local area-averaged Nusselt number ratio variations as dependent upon buoyancy parameter for the smooth surface rotating channel investigation of Huh et al. [104]. Region 4 is measured with radially outward flow, and region 12 is measured with radially inward flow.

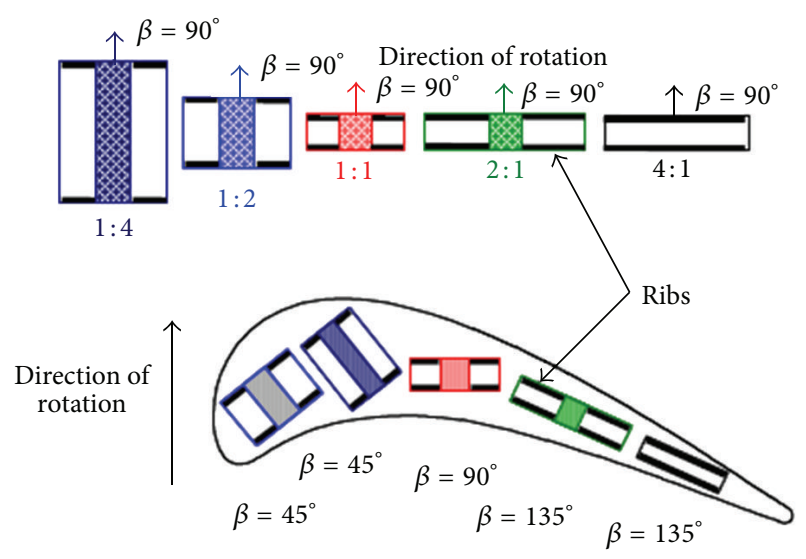

FIgURE 28: Examples of various channel orientations and aspect ratios employed for internal cooling of turbine blades from Fu et al. [128]. 


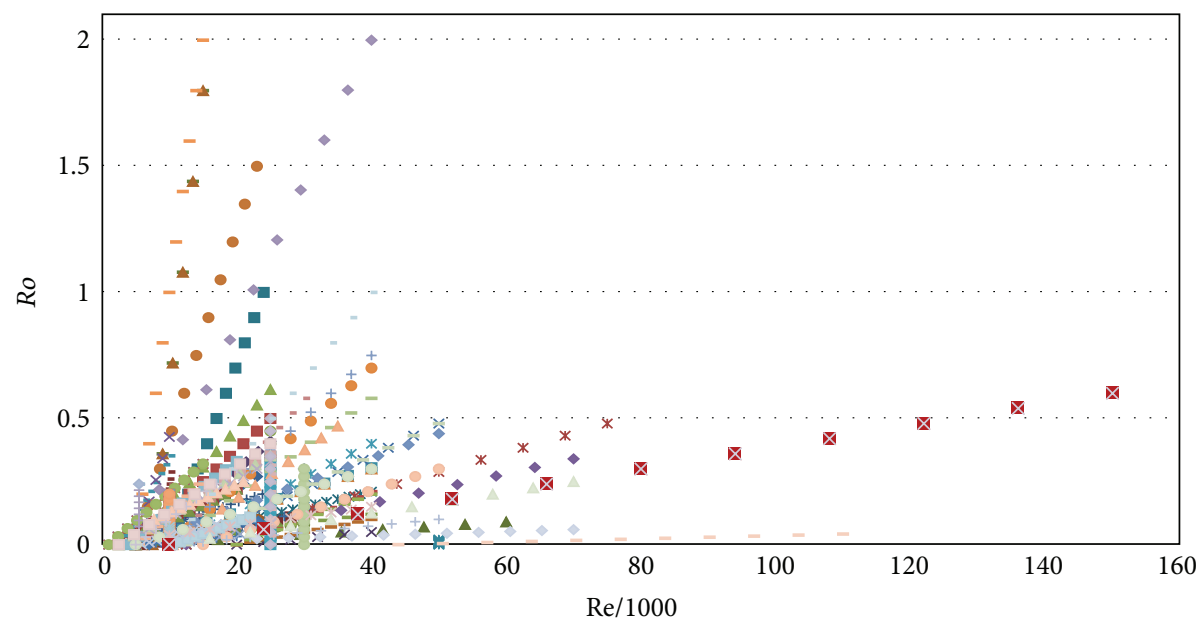

- Acharya et al. [238]

$\times$ Agarwal et al. [239]

+ Azad et al. [58]

- Chang and Morris [220]

$x$ Chang et al. [236]

+ Cho et al. [240]

- Dutta and Han [64]

$\times$ Dutta et al. [223]

+ Shin et al. [66]

- Fu et al. [187]

$\times$ Hajek and Higgins [188]

+ Han and Zhang [210]

- Harasgama and Morris [224]

$\times$ Hsieh et al. [192]
- Acharya et al. [180]

* Al-Hadhrami et al. [183]

- Bons and Kerrebrock [178]

- Chang et al. [221]

* Chen and Liou [101]

- Cho et al. [241]

- Dutta et al. [232]

* Elfert et al. [50]

- Fu et al. [186]

- Griffith et al. [208]

* Hajek et al. [189]

- Han et al. [190]

- Hsieh and Hong [225]

* Huh et al. [104]
A Acharya et al. [129]

- Al-Hadhrami and Han [184]

- Chang et al. [231]

- Chang et al. [222]

- Chiang and Li [185]

- Cho et al. [242]

- Dutta et al. [65]

- El-Husayni et al. [261]

- Fu et al. [128]

^ Griffith et al. [209]

- Hajek et al. [263]

- Han et al. [211]

4 Hsieh and Liu [191]

- Huh et al. [193]

(a)

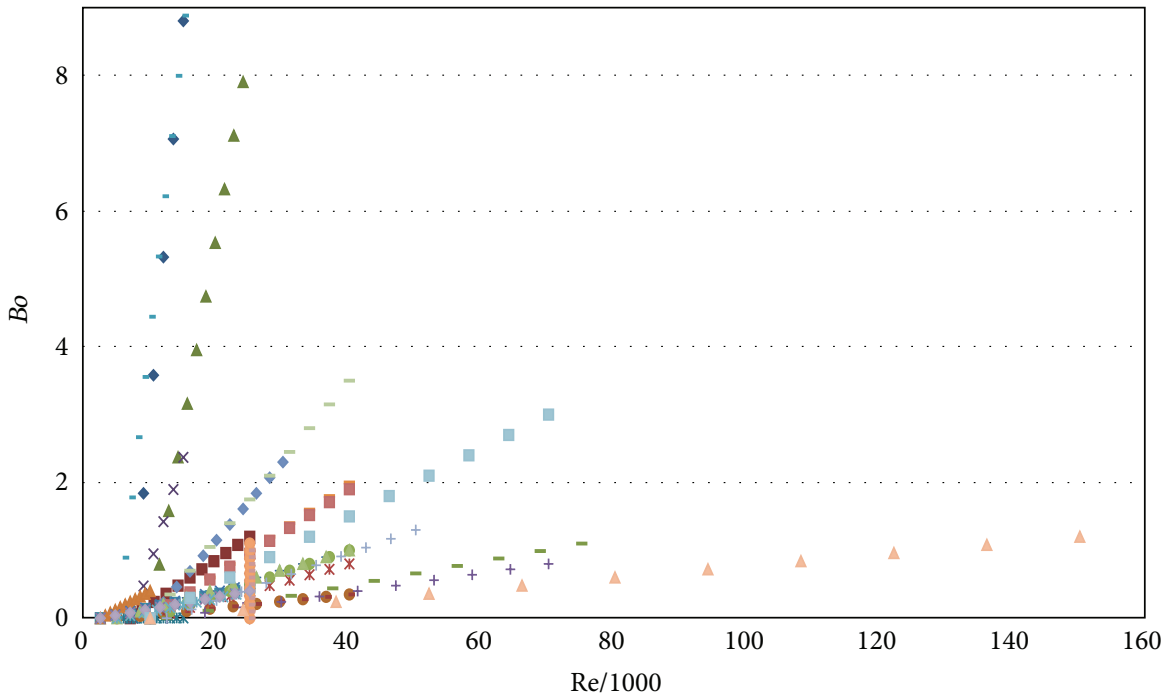

- Chang et al. [231]

- Fu et al. [186]

- Han and Zhang [210]

+ Johnson et al.[74]

L Liu et al. [182]

- Wagner et al. [204]

- Chang and Morris [220]

+ Fu et al. [128]

^ Han et al. [190]

- Liou et al. [233] $\times$ Parsons et al. [81]

- Wright et al. [230]

- Chang et al. [221]

- Fu et al. [187]

$\times$ Han et al. [211]

- Liu et al. [200]

* Servouze and Sturgis [177]

- Zhang et al. [100]

$\times$ Chang et al. [222]

- Hajek et al. [189]

(b)
* Huh et al. [104]

- Liu et al. [268]

- Wagner et al. [202, 203]

- Zhou and Acharya [102]

* Chang et al. [236]

- Hajek et al. [263]

- Huh et al. [193]

- Liu et al. [269]

+ Wagner et al. [103]

$\triangle$ Zhou et al. [206]

FIgURE 29: Continued. 

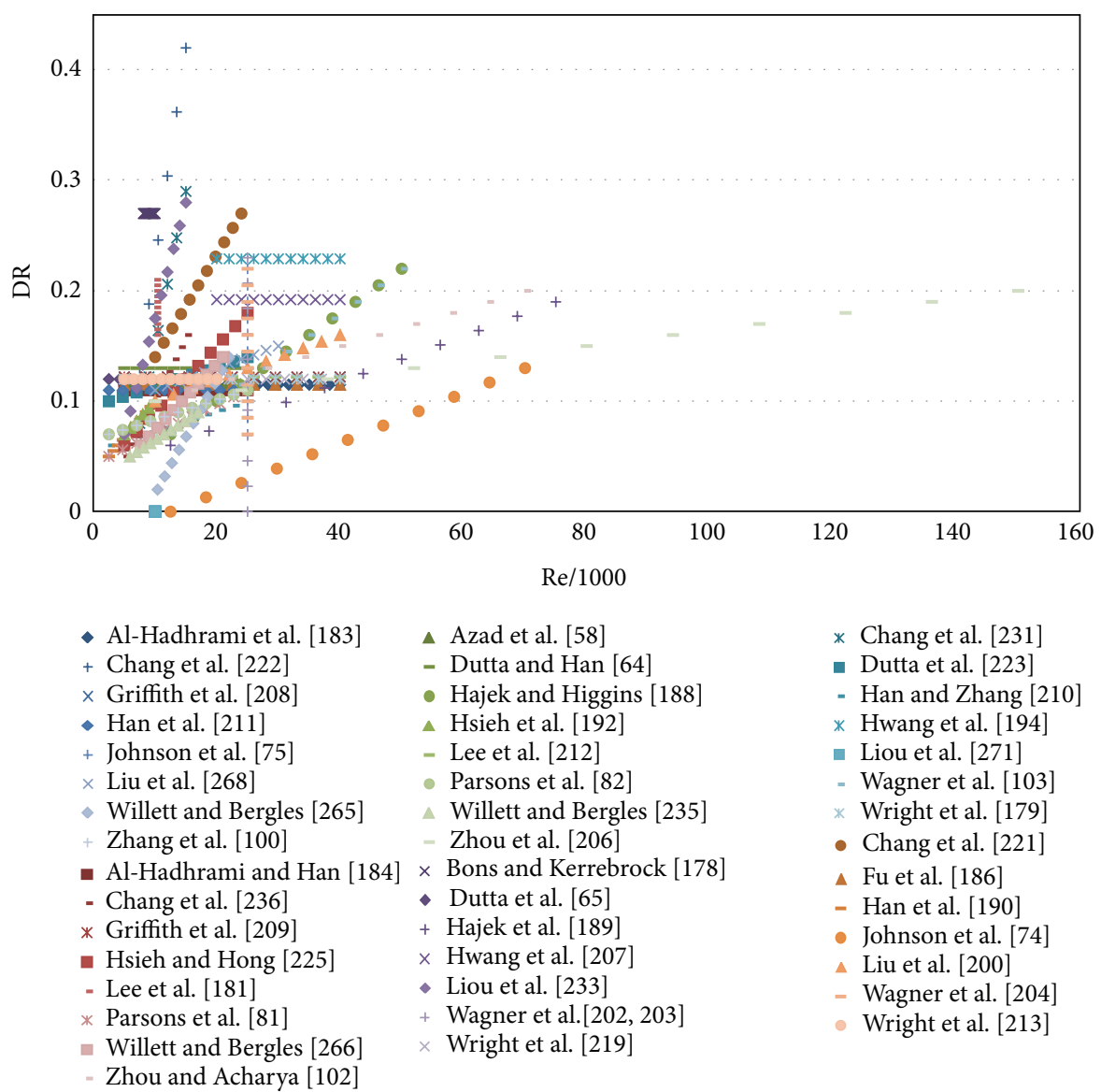

* Chang et al. [231]
- Dutta et al. [223]
- Han and Zhang [210]
* Hwang et al. [194]
- Liou et al. [271]
- Wagner et al. [103]
* Wright et al. [179]
- Chang et al. [221]
- Fu et al. [186]
- Han et al. [190]
- Johnson et al. [74]
- Liu et al. [200]
- Wagner et al. [204]
- Wright et al. [213]

(c)

FIGURE 29: Ranges of experimental parameters for existing investigations of channel flows with rotation. (a) Rotation number versus Reynolds number. (b) Buoyancy parameter versus Reynolds number. (c) Inlet density ratio versus Reynolds number.

combines the effects of rotation number, density ratio, and a geometric configuration parameter. With radially outward flow, Nusselt number ratios increase with buoyancy parameter on the passage pressure side and decrease with buoyancy parameter on the passage suction side. With radially inward flow, the converse is generally true, with suction side Nusselt numbers increasing with buoyancy parameter, such that values are generally larger than Nusselt numbers measured on the passage pressure side $[74,100,102-104,128,177,182$, $186,187,189,190,193,200,204,206,210,220-222,263$, 265, 267]. The channel cross-section for the Huh et al. [104] investigation has a $12.70 \mathrm{~mm}$ height, and $23.81 \mathrm{~mm}$ width, resulting in a hydraulic diameter of $16.93 \mathrm{~mm}$.

4.5. Effects of Channel Geometry and Orientation. In regard to aspect ratio, $\mathrm{Fu}$ et al. [128, 187] consider a variety of arrangements, and often show similar Nusselt number trends regardless of the aspect ratio, particularly as the buoyancy parameter is changed. In some situations, Nusselt number ratios are slightly higher in channels with low aspect ratios. In addition, pressure losses developing in low aspect ratios are generally significantly less than channels with higher aspect ratios $[128,187]$.
The results in Figure 28 illustrate the variety of different channel orientations which are sometimes employed within a turbine rotor blade. From these arrangements, the importance of channel orientation with respect to the rotating axis is evident because not all internal cooling channels are oriented perpendicular to the rotating axis. With the arrangement shown in Figure 21, pressure side is synonymous with trailing side, and suction side is synonymous with leading side. Within a $45^{\circ}$ oriented channel, for example, designating the suction side and pressure side is not longer so straight-forward. For example, the suction side can still be considered the leading side, or it can be considered to be a combination of a portion of the leading side, a sidewall, and even a small portion of the trailing side. According to several investigations $[64,180,183,184,187,209]$, rotation appears to have less influence on heat transfer on the leading and trailing sides when the channel is oriented at $45^{\circ}$, compared to a $90^{\circ}$ arrangement.

In regard to channel shape, triangular $[65,181,232,268$, 269] and circular [226, 270] ducts are also investigated. In regard to triangular arrangements, care is required when considering the pressure and suction sides of the rotating passages. Overall, rotation and buoyancy have less influence 


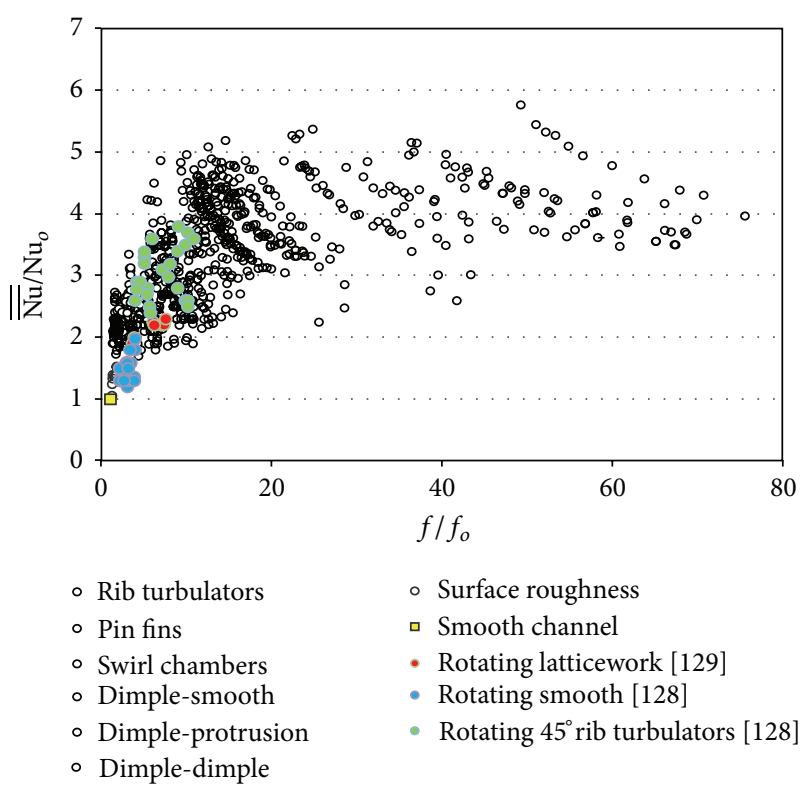

FIGURE 30: Comparisons of relative performance of rib turbulators, pin fins, swirl chambers, dimple-smooth arrangements, dimpleprotrusion arrangements, dimple-dimple arrangements, surface roughness, and smooth-walled channels [1], with results from rotation experiments $[128,173]$. Globally averaged Nusselt number ratios $\overline{\overline{\mathrm{Nu}}} / \mathrm{Nu}_{o}$ are presented as dependent upon friction factor ratios $f / f_{o}$.

on heat transfer in ribbed channels than smooth ducts [269]. In addition, different rib orientations and arrangements produce different Nusselt number ratio distributions, especially as either the rotation number or the buoyancy parameter increases [268].

4.6. Effects of Different Wall Heating Arrangements. Several studies examine the effects different wall heating arrangements. For example, El-Husayni et al. [261] apply uniform heat flux to several different rotating channel arrangements: the primary wall only, two opposite walls only, and all four walls together. All of the other non-heated surfaces are maintained adiabatic. Comparisons generally show that the different configurations result in minimal changes to magnitudes and distributions of local Nusselt number ratios. The only exception is an increase in Nusselt number ratio on the pressure side of a ribbed channel with all four walls at uniform heat flux as the rotation number increases. With other conditions and arrangements, Nusselt number ratios either remained constant or decreased. Han and Zhang [210] consider rotating channels with all four walls at the same temperature, four walls with uniform heat flux, and with the pressure side hotter than the suction side. The investigators conclude that heat transfer coefficients on the pressure side are higher with uniform heat flux than with uniform wall temperature. Heat transfer coefficients on the suction side are then much higher with uniform heat flux and hotter pressure side, than when a uniform wall temperature is imposed.
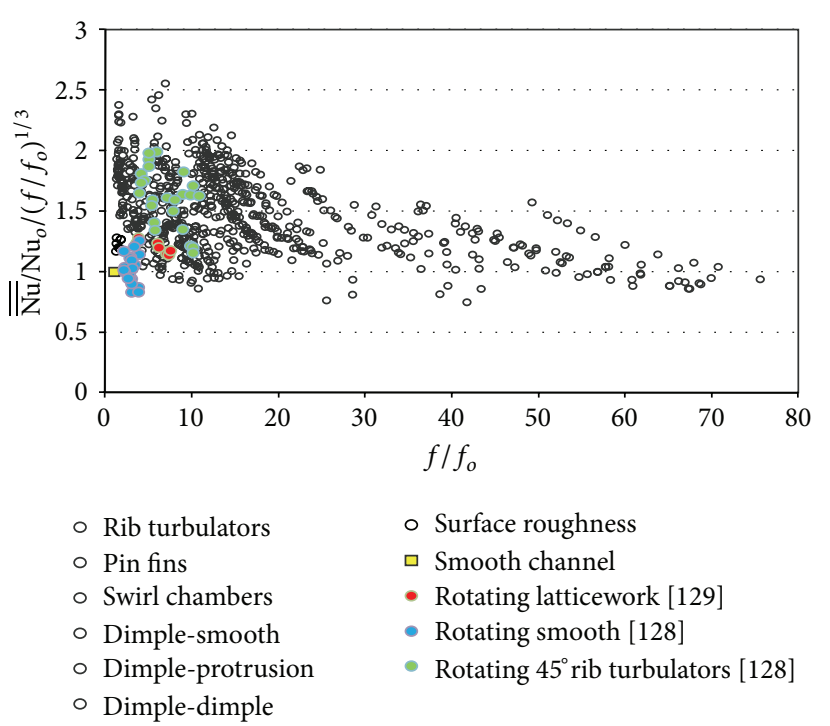

- Surface roughness

- Smooth channel

- Rotating latticework [129]

- Rotating smooth [128]

- Rotating $45^{\circ}$ rib turbulators [128]

- Dimple-protrusion

FIGURE 31: Comparisons of relative performance of rib turbulators, pin fins, swirl chambers, dimple-smooth arrangements, dimpleprotrusion arrangements, dimple-dimple arrangements, surface roughness, and smooth-walled channels [1], with results from rotation experiments $[128,173]$. Globally averaged thermal performance parameters $\overline{\overline{\mathrm{Nu}}} / \mathrm{Nu}_{o} /\left(f / f_{o}\right)^{1 / 3}$ are presented as dependent upon friction factor ratios $f / f_{o}$.

4.7. Ranges of Reynolds Number, Rotation Number, Buoyancy Parameter, and Inlet Density Ratio of Existing Investigations. Investigated ranges of Reynolds number, rotation number, buoyancy parameter, and inlet density ratio for existing investigations of rotating channel flows are shown in Figure 29. The overall objective of the related studies is application of experimental data to the operating conditions of turbine blades within industrial and aeroengine gas turbine engines. However, unfortunately, magnitudes of Reynolds number, rotation number, buoyancy parameter, and inlet density ratio are generally (in some manner) smaller than the actual values which are encountered in operating engines. For example, see investigation of Liou et al. [271].

One reason for these deficiencies is because high rotation numbers can be achieved by maintaining rotational speed and reducing the Reynolds number. As a result, rotation numbers within the range of operating turbines within gas turbine engines can be produced, but at the penalty of a low Reynolds number.

As indicated, local, spatially resolved heat transfer augmentations and reductions within rotating channels are dependent upon both Reynolds number and rotation number. As such, additional, high-quality experimental data are needed, with high Reynolds numbers simultaneously with high rotation numbers (to correctly match engine operating conditions), especially when these two parameters are varied independently of each other.

4.8. Rotation and Its Relationship to Thermal Performance Parameters. Here, comparisons are made between results presented by Ligrani et al. [1] for stationary (nonrotating) 


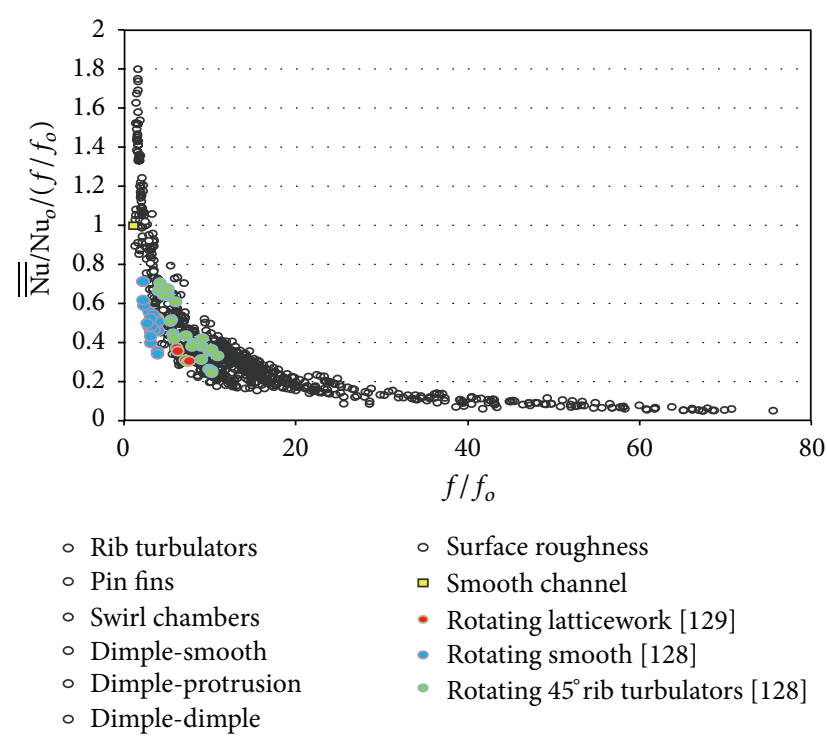

FIGURE 32: Comparisons of relative performance of rib turbulators, pin fins, swirl chambers, dimple-smooth arrangements, dimpleprotrusion arrangements, dimple-dimple arrangements, surface roughness, and smooth-walled channels [1], with results from rotation experiments $[128,173]$. Globally averaged thermal performance parameters $\overline{\overline{\mathrm{Nu}}} / \mathrm{Nu}_{o} /\left(f / f_{o}\right)$ are presented as dependent upon friction factor ratios $f / f_{o}$.

channels, and experimental thermal performance data from rotating channels $[128,173]$. As discussed previously, Ligrani et al. [1] present information on heat transfer augmentation techniques with respect to internal cooling channels, including rib turbulators, pin fins, dimpled surfaces, surfaces with protrusions, swirl chambers, and surface roughness. Included are spatially averaged thermal performance parameters as they relate to friction factor ratios. The comparisons between the rotating channel experimental data $[128,173]$ and the Ligrani et al. [1] results are given in Figures 30, 31, and 32. The rotating lattice work arrangement [173] is comprised of a crossing-rib configuration on leading and trailing passage sides to model a coolant passage with inclined serpentine subchannels. Each rib in this arrangement is inclined at 45 degrees relative to the radial direction, such that ribs on opposite walls are arranged with an aligned and crossing arrangement, such that the subchannel on one surface turns smoothly to the subchannel on the opposite surface. This arrangement forces the fluid within the latticework configuration to turn by 90 degrees in a repeated and periodic fashion. As shown by Figures 30, 31, and 32, the rotating thermal performance characteristics are distributed such that they are well within the scatter and distribution of the stationary channel performance data. This indicates that rotation has little effect on overall thermal performance as a function of friction factor. Additional confirmation is also provided that overall, spatially averaged performance characteristics from rotating channel studies are generally insensitive to variations of rotation number.

\section{Summary and Conclusions}

Many important constraint requirements are imposed upon the development of internal cooling configurations for gas turbine engines. Satisfying these constraints requires simultaneous consideration of multidisciplinary issues, such as manufacturing, airfoil surface cooling requirements, aerodynamic losses, coolant availability, second law losses, available volume space, airfoil shape, and pressure drop penalties, in addition to heat transfer and thermal considerations. As a result, current and future trends for advanced turbine component cooling design require simultaneous development of external thermal protection schemes, like film cooling, with internal cooling technologies, including consideration of the adjacent conjugate conduction variations in adjacent solid components, as well as associated aerodynamic pressure loss penalty issues. To meet these requirements, designers, manufacturers, and researchers must develop internal cooling systems with combinations of different devices together within one coolant passage. Resulting objectives are optimal thermal protection and minimal pressure loss penalties.

To provide an overview of the current state of the art of heat transfer augmentation schemes employed for internal cooling of turbine blades and components, results from an extensive literature review are presented with data from internal cooling channels, both with and without rotation. According to this survey, a very small number of existing investigations consider the use of combination devices for internal passage heat transfer augmentation. Examples are rib turbulators, pin fins and dimples together [4], a combination of pin fins and dimples [5], and rib turbulators and pin fins in combination [6]. The results of such studies are compared with data obtained prior to 2003 without rotation influences, as summarized by Ligrani et al. [1]. Those data [1] are comprised of heat transfer augmentation results for internal cooling channels, with rib turbulators, pin fins, dimpled surfaces, surfaces with protrusions, swirl chambers, or surface roughness.

This comparison reveals that all of the new data, obtained since 2003, are collected within the distribution of data obtained from investigations conducted prior to 2003, without rotation influences [1]. A newer arrangement with the most promising performance characteristics may be the rib investigation of Salameh and Sunden [155], since Nusselt number ratios are as high as 4.2 with a corresponding friction factor ratio of 7.8. The same conclusion in regard to data distributions is also reached in regard to globally averaged thermal performance parameters, $\overline{\overline{\mathrm{Nu}}} / \mathrm{Nu}_{o} /\left(f / f_{o}\right)^{1 / 3}$ and $\overline{\overline{\mathrm{Nu}}} / \mathrm{Nu}_{o} /\left(f / f_{o}\right)$, as they vary with friction factor ratio $f / f_{o}$. These comparisons, made on the basis of such judgement criteria, lead to the conclusion that improvements in our ability to provide better overall, spatially averaged thermal protection have been minimal since 2003. However, in spite of this conclusion, many heat transfer augmentation technologies, developed since 2003, may provide improved local thermal protection, compared to many devices developed prior to that time. Such local thermal protection is mostly 
related to local gradients of temperature and heat transfer, which are also vitally important for the long-term durability and reliability of gas turbine engines. Local temperature gradients are also important regarding local thermal stress magnitudes. The best configurations, with the lowest thermal stresses, are thus, the ones with the lowest local temperature gradients.

When rotation is present, existing investigations provide little evidence of overall increases or decreases in overall thermal performance characteristics with rotation, at any value of rotation number, buoyancy parameter, density ratio, or Reynolds number. In addition, overall thermal performance in smooth channels with rotation is affected more by Reynolds number than rotation number. Comparisons between the rotating channel experimental data $[128,173]$ and the Ligrani et al. [1] results show that rotating thermal performance characteristics are distributed well within the distribution of the stationary performance data, obtained prior to 2003. This also indicates that rotation has little effect on overall thermal performance as a function of friction factor. Additional confirmation is also provided that overall, spatially averaged performance characteristics from rotating channel studies are generally insensitive to variations of rotation number.

This is largely because of the competing effects of rotation on the pressure (or trailing) sides and the suction (or leading) sides of internal channel flows. Differences in local Nusselt number ratios for pressure sides and suction sides are generally a result of increasing rotation numbers, and the rotation induced secondary flows caused by Coriolis vortices. For radially outward flow channels, the effects of these secondary flows produce local heat transfer augmentations on the pressure (or trailing) sides of channels, and local heat transfer decreases on suction (or leading) sides (compared to similar channels with no rotation). Different trends with increasing rotation number are present for the two sides of a rotating channel with radially inward flow.

Local area-averaged Nusselt number ratios are affected by variations of the buoyancy parameter in the same approximate qualitative manner as when rotation number changes. With radially outward flow, Nusselt number ratios increase with buoyancy parameter on the passage pressure side, and decrease with buoyancy parameter on the passage suction side (compared to similar channels with no rotation). When the inlet density ratio is altered, results for a radial outflow arrangement show that increasing this parameter enhances the Nusselt number ratio for both pressure and suction side surfaces over a range of rotation numbers.

To further advance heat transfer augmentation technologies for internal cooling of turbine components of gas turbine engines, additional high quality experimental data are needed. Each experimental requires complete documentation of relevant characteristics, including local and spatially averaged distributions of surface Nusselt numbers, as well as overall flow passage pressure drop penalties and friction factor ratios. Relevant engine operating conditions, both with and without rotation, must be matched whenever possible. Additional thermal performance improvements can be obtained with singular devices utilizing detailed and extensive knowledge of the effects and interactions between geometry and configuration details, and the resulting flow and heat transfer characteristics. Use of novel combination devices also offers excellent opportunities for improved internal cooling thermal performance. New high quality rotation experimental data are additionally needed (especially, local, spatially resolved data), particularly with different inflow and outflow arrangements, and a variety of heat transfer augmentation devices.

\section{Nomenclature}

Bo: Buoyancy parameter

D: Dimple print diameter

$D$ : Channel length scale

$D$ : $\quad$ Pin fin length scale

$D_{h}$ : Channel or duct hydraulic diameter

$D R$ : Inlet density ratio, $(\Delta \rho / \rho)_{i}$

$f: \quad$ Friction factor

$f_{o}$ : Baseline friction factor in a smooth channel with no augmentation devices

$H$ : Channel length scale

$H$ : Dimple depth

$\mathrm{Nu}$ : Local Nusselt number based on channel length scale

$\mathrm{Nu}_{o}$ : Baseline, constant property Nusselt number in a smooth channel with no augmentation devices

$\mathrm{Nu}_{S}$ : Baseline, constant property Nusselt number in a smooth channel with no augmentation devices

$R$ : Rotation radial position

Re: Reynolds number based on channel length scale and channel spatially-averaged inlet velocity

Ro: Rotation number

T: Temperature

$U$ : Channel or duct spatially-averaged inlet velocity

$x$ : Longitudinal coordinate

$X$ : Longitudinal coordinate

$\bar{X}$ : Longitudinal coordinate

$X^{*}$ : Normalized longitudinal coordinate

$y$ : Normal coordinate

$Y^{*}:$ Normalized normal coordinate

$z$ : Spanwise coordinate.

\section{Greek Symbols}

$\beta$ : Rotation angle

$\rho$ : Density

$\nu$ : Kinematic viscosity

$\Omega$ : Rotational angular velocity. 


\section{Superscripts}

=: Globally averaged value.

\section{Subscripts}

$c$ : Coolant stagnation value

$w$ : Wall value.

\section{References}

[1] P. M. Ligrani, M. M. Oliveira, and T. Blaskovich, "Comparison of heat transfer augmentation techniques," AIAA Journal, vol. 41, no. 3, pp. 337-362, 2003.

[2] J. C. Han, S. Dutta, and S. Ekkad, Gas Turbine Heat Transfer and Cooling Technology, Taylor and Francis, New York, NY, USA, 2000.

[3] J. C. Han, J. S. Park, and C. K. Lie, "Heat Transfer and pressure drop in blade cooling channels with turbulence promoters," NASA CR-3837, 1984.

[4] A. Murata, S. Nishida, H. Saito, K. Iwamoto, Y. Okita, and C. Nakamata, "Heat transfer enhancement due to combination of dimples, protrusions, and ribs in narrow internal passage of gas turbine blade," ASME Paper GT2011-45356, 2011.

[5] Y. Rao, C. Wan, Y. Xu, and S. Zang, "Local heat transfer characteristics in channels with pin fin and pin fin-dimple arrays," ASME Paper GT2011-45449, 2011.

[6] S. C. Siw, M. K. Chyu, and M. A. Alvin, "Effects of pin detached space on heat transfer in a rib roughened channel," ASME Paper GT2011-46078, 2011.

[7] B. A. Brigham and G. J. VanFossen, "Length to diameter ratio and row number effects in short pin fin heat transfer," Journal of Engineering for Gas Turbines and Power, vol. 106, no. 1, pp. 241-245, 1984.

[8] P. M. Ligrani and G. I. Mahmood, "Variable property Nusselt numbers in a channel with pin fins," Journal of Thermophysics and Heat Transfer, vol. 17, no. 1, pp. 103-111, 2003.

[9] S. C. Siw, M. K. Chyu, and M. A. Alvin, "Heat transfer enhancement of internal cooling passage with triangular and semi-circular shaped pin-fin arrays," ASME Paper GT201269266, 2012.

[10] B. Sundén, G. Xie, and Q. Wang, "Predictions of enhanced heat transfer of an internal blade tip-wall with hemispherical dimples or protrusions," ASME Paper GT2010-22265, 2010.

[11] W. Chen, J. Ren, and H. Jiang, "Effect of turning vane configurations on heat transfer and pressure drop in a ribbed internal cooling system," ASME Paper GT2010-22273, 2010.

[12] D. E. Metzger, R. A. Barry, and J. P. Bronson, "Developing heat transfer in rectangular ducts with staggered arrays of short pinfins," Journal of Heat Transfer, vol. V 104, no. 4, pp. 700-706, 1982.

[13] D. E. Metzger and S. W. Haley, "Heat transfer experiments and flow visualization for arrays of short pin-fins," ASME Paper 82GT-138, 1982.

[14] E. M. Sparrow, J. W. Ramsey, and C. A. C. Altemani, "Experiments on in-line pin fin arrays and performance comparisons with staggered array," Journal of Heat Transfer, vol. 102, no. 1, pp. 44-50, 1980.
[15] A. Zukauskas, "Heat transfer from tubes in cross flow," Advances in Heat Transfer, vol. 8, pp. 96-160, 1972.

[16] G. J. VanFossen, "Heat-transfer coefficients for staggered arrays of short pin-fins," Journal of Engineering for Power, vol. 104, no. 2, pp. 268-274, 1982.

[17] R. J. Simoneau and G. J. VanFossen, "Effects of location in an array on heat transfer to a short cylinder in cross flow," Journal of Heat Transfer, vol. 106, no. 1, pp. 42-48, 1984.

[18] S. C. Lau, J. C. Han, and Y. S. Kim, “Turbulent heat transfer and friction in pin fin channels with lateral flow ejection," Journal of Heat Transfer, vol. 111, no. 1, pp. 51-58, 1989.

[19] R. D. McMillin and S. C. Lau, "Effect of trailing-edge ejection on local heat (mass) transfer in pin fin cooling channels in turbine blades," Journal of Turbomachinery, vol. 116, no. 1, pp. 159-168, 1994.

[20] M. K. Chyu, "Heat transfer and pressure drop for short pin-fin arrays with pin-endwall fillet," Journal of Heat Transfer, vol. 112, no. 4, pp. 926-932, 1990.

[21] M. K. Chyu and R. J. Goldstein, "Influence of an array of wallmounted cylinders on the mass transfer from a flat surface," International Journal of Heat and Mass Transfer, vol. 34, no. 9, pp. 2175-2186, 1991.

[22] M. K. Chyu, Y. C. Hsing, T. I. P. Shih, and V. Natarajan, "Heat transfer contributions of pins and endwall in pin-fin arrays: effects of thermal boundary condition modeling," Journal of Turbomachinery, vol. 121, no. 2, pp. 257-263, 1999.

[23] V. B. Grannis and E. M. Sparrow, "Numerical simulation of fluid flow through an array of diamond-shaped pin fins," Numerical Heat Transfer A, vol. 19, no. 4, pp. 381-403, 1991.

[24] D. A. Olson, "Heat transfer in thin, compact heat exchangers with circular, rectangular, or pin-fin flow passages," Journal of Heat Transfer, vol. 114, no. 2, pp. 373-382, 1992.

[25] M. K. Chyu, Y. C. Hsing, and V. Natarajan, "Convective heat transfer of cubic fin arrays in a narrow channel," Journal of Turbomachinery, vol. 120, no. 2, pp. 362-367, 1998.

[26] M. K. Chyu and V. Natarajan, "Heat transfer on the base surface of three-dimensional protruding elements," International Journal of Heat and Mass Transfer, vol. 39, no. 14, pp. 2925-2935, 1996.

[27] J. J. Hwang and C. C. Lu, "Lateral-flow effect on endwall heat transfer and pressure drop in a pin-fin trapezoidal duct of various pin shapes," ASME Paper 2000-GT-232, 2000.

[28] O. Uzol and C. Camci, "Elliptical pin fins as an alternative to circular pin fins for gas turbine blade cooling applications. Part 1: endwall heat transfer and total pressure loss characteristics," ASME Paper 2001-GT-180, 2001.

[29] O. Uzol and C. Camci, "Elliptical pin fins as an alternative to circular pin fins for gas turbine blade cooling applications. Part 2: wake flow field measurements and visualization using particle image velocimetry," ASME Paper 2001-GT-181, 2001.

[30] S. Y. Won, G. I. Mahmood, and P. M. Ligrani, "Spatially-resolved heat transfer and flow structure in a rectangular channel with pin fins," International Journal of Heat and Mass Transfer, vol. 47, no. 8-9, pp. 1731-1743, 2004.

[31] V. N. Murzin, S. A. Stoklitskii, and A. P. Chebotarev, "Creation of solitary vortices in a flow around shallow spherical depressions," Soviet Technical Physical Letters, vol. 12, no. 11, pp. 547-549, 1986.

[32] P. R. Gromov, A. B. Zobnin, M. I. Rabinovich, and M. M. Sushchik, "Creation of solitary vortices in a flow around shallow spherical depressions," Soviet Technical Physical Letters, vol. 12, no. 11, pp. 1323-1328, 1986. 
[33] V. S. Kesarev and A. P. Kozlov, "Convective heat transfer in turbulized flow past a hemispherical cavity," Heat Transfer Research, vol. 25, no. 2, pp. 156-160, 1993.

[34] V. N. Afanasyev, Y. P. Chudnovsky, A. I. Leontiev, and P. S. Roganov, "Turbulent flow friction and heat transfer characteristics for spherical cavities on a flat plate," Experimental Thermal and Fluid Science, vol. 7, no. 1, pp. 1-8, 1993.

[35] M. Y. Belen'kiy, M. A. Gotovskiy, B. M. Lekakh, B. S. Fokin, and K. S. Dolgushin, "Heat transfer augmentation using surfaces formed by a system of spherical cavities," Heat Transfer Research, vol. 25, no. 2, pp. 196-203, 1993.

[36] V. I. Terekhov, S. V. Kalinina, and Y. M. Mshvidobadze, "Flow structure and heat transfer on a surface with a unit hole depression," Russian Journal of Engineering Thermophysics, vol. 5, pp. 11-34, 1995.

[37] V. D. Zhak, "The taylor-goertler vortices and three-dimensional flow evolution in a cavity," Russian Journal of Engineering Thermophysics, vol. 5, pp. 165-176, 1995.

[38] A. V. Schukin, A. P. Kozlov, and R. S. Agachev, "Study and application of hemispheric cavities for surface heat transfer augmentation," ASME Paper 95-GT-59, 1995.

[39] P. W. Bearman and J. K. Harvey, "Control of circular cylinder flow by the use of dimples," AIAA Journal, vol. 31, no. 10, pp. 1753-1756, 1993.

[40] T. Kimura and M. Tsutahara, "Fluid dynamic effects of grooves on circular cylinder surface," AIAA Journal, vol. 29, no. 12, pp. 2062-2068, 1991.

[41] M. E. Kithcart and D. E. Klett, "Heat transfer and skin friction comparison of dimpled versus protrusion roughness," Journal of Enhanced Heat Transfer, vol. 3, no. 4, pp. 273-280, 1996.

[42] M. K. Chyu, Y. Yu, H. Ding, J. P. Downs, and F. O. Soechting, "Concavity enhanced heat transfer in an internal cooling passage," ASME Paper 97-GT-437, 1997.

[43] Y. L. Lin, T. I.-P. Shih, and M. K. Chyu, "Computations of flow and heat transfer in a channel with rows of hemispherical cavities," ASME Paper 99-GT-263, 1999.

[44] Y. F. Gortyshov, I. A. Popov, R. D. Amirkhanov, and K. E. Gulitsky, "Studies of hydrodynamics and heat exchange in channels with various types of intensifiers," in Proceedings of 11th International Heat Transfer Congress (IHTC '98), vol. 6, pp. 8388, 1998.

[45] H. K. Moon, T. O’Connell, and B. Glezer, "Channel height effect on heat transfer and friction in a dimpled passage," ASME Paper 99-GT-163, 1999.

[46] J. Chen, H. Müller-Steinhagen, and G. G. Duffy, "Heat transfer enhancement in dimpled tubes," Applied Thermal Engineering, vol. 21, no. 5, pp. 535-547, 2001.

[47] S. W. Moon and S. C. Lau, "Turbulent heat transfer measurements on a wall with concave and cylindrical dimples in a square channel," ASME Paper GT-2002-30208, 2002.

[48] G. I. Mahmood, M. L. Hill, D. L. Nelson, P. M. Ligrani, H. K. Moon, and B. Glezer, "Local heat transfer and flow structure on and above a dimpled surface in a channel," Journal of Turbomachinery, vol. 123, no. 1, pp. 115-123, 2001.

[49] G. I. Mahmood and P. M. Ligrani, "Heat transfer in a dimpled channel: combined influences of aspect ratio, temperature ratio, Reynolds number, and flow structure," International Journal of Heat and Mass Transfer, vol. 45, no. 10, pp. 2011-2020, 2002.

[50] M. Elfert, M. Voges, and J. Klinner, "Detailed flow investigation using PIV in a rotating square-sectioned two-pass cooling system with ribbed walls," ASME Paper GT2008-51183, 2008.
[51] N. K. Burgess and P. M. Ligrani, "Effects of dimple depth on channel nusselt numbers and friction factors," Journal of Heat Transfer, vol. 127, no. 8, pp. 839-847, 2005.

[52] P. M. Ligrani, J. L. Harrison, G. I. Mahmmod, and M. L. Hill, "Flow structure due to dimple depressions on a channel surface," Physics of Fluids, vol. 13, no. 11, pp. 3442-3451, 2001.

[53] G. I. Mahmood, M. Z. Sabbagh, and P. M. Ligrani, "Heat transfer in a channel with dimples and protrusions on opposite walls," Journal of Thermophysics and Heat Transfer, vol. 15, no. 3, pp. 275-283, 2001.

[54] P. M. Ligrani, G. I. Mahmood, J. L. Harrison, C. M. Clayton, and D. L. Nelson, "Flow structure and local Nusselt number variations in a channel with dimples and protrusions on opposite walls," International Journal of Heat and Mass Transfer, vol. 44, no. 23, pp. 4413-4425, 2001.

[55] S. A. Isaev, A. I. Leont'ev, and P. A. Baranov, "Identification of self-organized vortexlike structures in numerically simulated turbulent flow of a viscous incompressible liquid streaming around a well on a plane," Technical Physics Letters, vol. 26, no. 1, pp. 15-18, 2000.

[56] M. Al-Qahtani, H.-C. Chen, and J. C. Han, "A numerical study of flow and heat transfer in rotating rectangular channels $(\mathrm{AR}=4)$ with $45^{\circ} \mathrm{Rib}$ turbulators by reynolds stress turbulence model," ASME Paper GT-2002-30216, 2002.

[57] M. Al-Qahtani, Y. Jang, H. C. Chen, and J. C. Han, "Prediction of flow and heat transfer in rotating two-pass rectangular channels with $45^{\circ}$ Rib turbulators," ASME Paper 2001-GT-187, 2001.

[58] G. S. Azad, M. J. Uddin, J. C. Han, H. K. Moon, and B. Glezer, "Heat transfer in a two-pass rectangular rotating channel with 45-deg angleb rib turbulators," Journal of Turbomachinery, vol. 124, no. 2, pp. 251-259, 2002, ASME Paper 2001-GT-186.

[59] B. Bonhoff, S. Parneix, J. Leusch, B. V. Johnson, J. Schabacker, and A. Bölcs, "Experimental and numerical study of developed flow and heat transfer in coolant channels with 45 degree ribs," International Journal of Heat and Fluid Flow, vol. 20, no. 3, pp. 311-319, 1999.

[60] B. Bonhoff, U. Tomm, B. V. Johnson, and I. Jennions, "Heat transfer predictions for rotating U-shaped coolant channels with skewed ribs and with smooth walls," ASME Paper 97-GT162, 1997.

[61] R. S. Bunker, T. G. Wetzel, and D. L. Rigby, "Heat transfer in a complex trailing edge passage for a high pressure turbine blade-part 1: experimental measurements," ASME Paper GT2002-30212, 2002.

[62] L. Casarsa, M. Cakan, and T. Arts, "Characterization of the velocity and heat transfer fields in an internal cooling channel with high blockage ratio," ASME Paper GT-2002-30207, 2002.

[63] H. H. Cho, S. Y. Lee, and S. J. Wu, "The combined effects of rib arrangements and discrete ribs on local heat/mass transfer in a square duct," ASME Paper 2001-GT-175, 2001.

[64] S. Dutta and J. C. Han, "Local heat transfer in rotating smooth and ribbed two-pass square channels with three channel orientations," Journal of Heat Transfer, vol. 118, no. 3, pp. 578-584, 1996.

[65] S. Dutta, J. C. Han, and C. P. Lee, "Local heat transfer in a rotating two-pass ribbed triangular duct with two model orientations," International Journal of Heat and Mass Transfer, vol. 39, no. 4, pp. 707-715, 1996.

[66] F. Shin, Y. Wen-Jei, and Z. Nengli, "Local heat transfer in a rotating serpentine passage with rib-roughened surfaces," International Journal of Heat and Mass Transfer, vol. 37, no. 2, pp. 217-228, 1994. 
[67] T. S. Griffith, L. Al-Hadhrami, and J. C. Han, "Heat transfer in rotating rectangular cooling channels $(\mathrm{AR}=4)$ with angled ribs," Journal of Heat Transfer, vol. 124, no. 4, pp. 617-625, 2002.

[68] J. C. Han, L. R. Glicksman, and W. M. Rohsenow, "An investigation of heat transfer and friction for rib-roughened surfaces," International Journal of Heat and Mass Transfer, vol. 21, no. 8, pp. 1143-1156, 1978.

[69] J. C. Han, J. J. Huang, and C. P. Lee, "Augmented heat transfer in square channels with wedge-shaped and delta-shaped turbulence promoters," Journal of Enhanced Heat Transfer, vol. 1, no. 1, pp. 37-52, 1993.

[70] J. C. Han and J. S. Park, "Developing heat transfer in rectangular channels with rib turbulators," International Journal of Heat and Mass Transfer, vol. 31, no. 1, pp. 183-195, 1988.

[71] J. C. Han, Y. M. Zhang, and C. P. Lee, "Augmented heat transfer in square channels with parallel, crossed, and V-shaped angled ribs," Journal of Heat Transfer, vol. 113, no. 3, pp. 590-596, 1991.

[72] H. Iacovides, "Computation of flow and heat transfer through rotating ribbed passages," International Journal of Heat and Fluid Flow, vol. 19, no. 5, pp. 393-400, 1998.

[73] R. Jia, A. Saidi, and B. Sunden, "Heat transfer enhancement in square ducts with V-shaped Ribs of various angles," ASME Paper GT-2002-30209, 2002.

[74] B. V. Johnson, J. H. Wagner, G. D. Steuber, and F. C. Yeh, "Heat transfer in rotating serpentine passages with trips skewed to the flow," Journal of Turbomachinery, vol. 116, no. 1, pp. 113-123, 1994, ASME Paper 92-GT-191.

[75] B. V. Johnson, J. H. Wagner, G. D. Steuber, and F. C. Yeh, "Heat transfer in rotating serpentine passages with selected model orientations for smooth or skewed trip walls," Journal of Turbomachinery, vol. 116, no. 4, pp. 738-744, 1994, ASME Paper 93-GT-305.

[76] Y. L. Lin, T. I. P. Shih, M. A. Stephens, and M. K. Chyu, "A numerical study of flow and heat transfer in a smooth and ribbed u-duct with and without rotation," Journal of Heat Transfer, vol. 123, no. 2, pp. 219-232, 2001.

[77] T. M. Liou, M. Y. Chen, and Y. M. Wang, "Heat transfer, fluid flow, and pressure measurements inside a rotating two-pass duct with detached 90-deg ribs," Journal of Turbomachinery, vol. 125, no. 3, pp. 565-574, 2003, ASME Paper GT-2002-30201.

[78] G. I. Mahmood, P. M. Ligrani, and S. Y. Won, "Spatially-resolved heat transfer and flow structure in a rectangular channel with $45^{\circ}$ angled rib turbulators," ASME Paper GT-2002-30215, 2002.

[79] C. W. Park, S. C. Lau, and R. T. Kukreja, "Effect of rib size on heat (Mass) transfer distribution in a rotating channel," ASME Paper 97-AA-126, 1997.

[80] C. W. Park, S. C. Lau, and R. T. Kukreja, "Heat (mass) transfer in a rotating channel with ribs of various sizes on two walls," Journal of Thermophysics and Heat Transfer, vol. 12, no. 3, pp. 452-454, 1998.

[81] J. A. Parsons, J. C. Han, and Y. M. Zhang, "Wall heating effect on local heat transfer in a rotating two-pass square channel with $90^{\circ}$ rib turbulators," International Journal of Heat and Mass Transfer, vol. 37, no. 9, pp. 1411-1420, 1994.

[82] J. A. Parsons, J. C. Han, and Y. Zhang, "Effect of model orientation and wall heating condition on local heat transfer in a rotating two-pass square channel with rib turbulators," International Journal of Heat and Mass Transfer, vol. 38, no. 7, pp. 1151-1159, 1995.

[83] S. V. Prabhu and R. P. Vedula, "Pressure drop distribution in a rotating rectangular channel with one ribbed surface," ASME Paper 97-AA-118, 1997.
[84] D. L. Rigby, "Prediction of heat and mass transfer in a rotating ribbed coolant passage with a 180 degree turn," ASME Paper 98GT-329, 1998.

[85] D. L. Rigby and R. S. Bunker, "Heat transfer in a complex trailing edge passage for a high pressure turbine blade-part 2: simulation results," ASME Paper GT-2002-30213, 2002.

[86] D. L. Rigby, E. Steinthorsson, and A. A. Ameri, "Numerical prediction of heat transfer in a channel with ribs and bleed," ASME Paper 97-GT-431, 1997.

[87] J. Schabacker, A. Bolcs, and B. V. Johnson, "PIV investigation of the flow characteristics in an internal coolant passage with 45 rib arrangement," ASME Paper 99-GT-120, 1999.

[88] T. I.-P. Shih, Y.-L. Lin, M. A. Stephens, and M. K. Chyu, "Flow and heat transfer in a ribbed U-duct under typical engine conditions," ASME Paper 98-GT-213, 1998.

[89] M. A. Stephens, M. K. Chyu, and T. I.-P. Shih, "Computations of convective heat transfer in a square duct with inclined ribs of rounded cross section," ASME Paper 96-WA/HT-12, 1996.

[90] M. A. Stephens and T. I.-P. Shih, "Computation of compressible flow and heat transfer in a rotating duct with inclined ribs and 180 degree bend," ASME Paper 97-GT-192, 1997.

[91] M. E. Taslim, L. A. Bondi, and D. M. Kercher, "Experimental investigation of heat transfer in an orthogonally rotating channel roughened with 45 deg criss-cross ribs on two opposite walls," Journal of Turbomachinery, vol. 113, no. 3, pp. 346-353, 1991, ASME Paper 90-GT-332.

[92] M. E. Taslim, T. Li, and D. M. Kercher, "Experimental heat transfer and friction in channels roughened with angled, Vshaped, and discrete ribs on two opposite walls," Journal of Turbomachinery, vol. 118, no. 1, pp. 20-28, 1996.

[93] M. E. Taslim, T. Li, and S. D. Spring, "Measurements of heat transfer coefficients and friction factors in passages ribroughened on all walls," Journal of Turbomachinery, vol. 120, no. 3, pp. 564-570, 1998.

[94] M. E. Taslim and A. Lengkong, “ $45^{\circ}$ staggered rib heat transfer coefficient measurements in a square channel," Journal of Turbomachinery, vol. 120, no. 3, pp. 571-580, 1998.

[95] M. E. Taslim, A. Rahman, and S. D. Spring, "Experimental investigation of heat transfer coefficients in a spanwise rotating channel with two opposite rib-roughened walls," Journal of Turbomachinery, vol. 113, no. 1, pp. 75-82, 1991, ASME Paper 89GT-150.

[96] M. E. Taslim and C. M. Wadsworth, "An experimental investigation of the rib surface-averaged heat transfer coefficient in a rib-roughened square passage," Journal of Turbomachinery, vol. 119, no. 2, pp. 381-389, 1997.

[97] D. Thurman and P. Poinsatte, "Experimental heat transfer and bulk air temperature measurements for a multipass internal cooling model with ribs and bleed," ASME Paper 2000-GT-233, 2000.

[98] D. G. N. Tse and G. D. Steubner, "Flow in a rotating square serpentine coolant passage with skewed trips," ASME Paper 97GT-529, 1997.

[99] K. Watanabe and T. Takahashi, "LES simulation and experimental measurement of fully developed ribbed channel flow and heat transfer," ASME Paper GT-2002-30203, 2002.

[100] Y. M. Zhang, J. C. Han, J. A. Parsons, and C. P. Lee, "Surface heating effect on local heat transfer in a rotating two-pass square channel with $60 \mathrm{deg}$ angled rib turbulators," Journal of Turbomachinery, vol. 117, no. 2, pp. 272-280, 1995, ASME Paper 93-GT-336. 
[101] C. C. Chen and T. M. Liou, "Rotating effect on fluid flow in a smooth duct with a 180-deg sharp turn," ASME Paper 2000-GT0228, 2000.

[102] F. Zhou and S. Acharya, "Heat transfer at high rotation numbers in a two-pass 4:1 aspect ratio rectangular channel with $45 \mathrm{deg}$ skewed ribs," Journal of Turbomachinery, vol. 130, no. 2, Article ID 021019, 2008.

[103] J. H. Wagner, B. V. Johnson, and T. J. Hajek, "Heat transfer in rotating passages with smooth walls and radial outward flow," Journal of Turbomachinery, vol. 113, no. 1, pp. 42-51, 1991, ASME Paper 89-GT-272.

[104] M. Huh, J. Lei, Y. H. Liu, and J. C. Han, "High rotation number effects on heat transfer in a rectangular $(\mathrm{AR}=2: 1)$ two pass channel," ASME Paper GT2009-59421, 2009.

[105] A. Ogawa, Vortex Flow, CRC Press, New York, NY, USA, 1993.

[106] B. Glezer, H.-K. Moon, and T. O'Connell, "A novel technoique for the internal blade cooling," ASME Paper 96-GT-181, 1996.

[107] B. Glezer, H.-K. Moon, J. Kerrebrock, J. Bons, and G. Guenette, "Heat transfer in a rotating radial channel with swirling internal flow," ASME Paper 98-GT-214, 1998.

[108] A. E. Bergles, "Survey and evaluation of techniques to augment convective heat and mass transfer," International Journal of Heat and Mass Transfer, vol. 1, pp. 331-413, 1969.

[109] H. H. Bruun, A. Fitouri, and M. K. Khan, "Use of a multiposition single yawed hot-wire probe for measurements in swirling flow," in Proceedings of the Fluids Engineering Conference, vol. 167, pp. 57-65, June 1993.

[110] F. Chang and V. K. Dhir, "Turbulent flow field in tangentially injected swirl flows in tubes," International Journal of Heat and Fluid Flow, vol. 15, no. 5, pp. 346-356, 1994.

[111] A. W. Date, "Prediction of fully-developed flow in a tube containing a twisted-tape," International Journal of Heat and Mass Transfer, vol. 17, no. 8, pp. 845-859, 1974.

[112] M. Dong and D. G. Lilley, "Parameter effects on flow patterns in confined turbulent swirling flows," in Proceedings of the International Joint Power Generation Conference, vol. 17, October 1993.

[113] A. Fitouri, M. K. Khan, and H. H. Bruun, "A multiposition hot-wire technique for the study of swirling flows in vortex chambers," Experimental Thermal and Fluid Science, vol. 10, no. 1, pp. 142-151, 1995.

[114] W. R. Gambill and R. D. Bundy, "An evaluation of the present status of swirl-flow heat transfer," ASME Paper 62-HT-42, 1962.

[115] C. R. Hedlund and P. M. Ligrani, "Local swirl chamber heat transfer and flow structure at different Reynolds numbers," Journal of Turbomachinery, vol. 122, no. 2, pp. 375-385, 2000.

[116] C. R. Hedlund, P. M. Ligrani, H. K. Moon, and B. Glezer, "Heat transfer and flow phenomena in a swirl chamber simulating turbine blade internal cooling," Journal of Turbomachinery, vol. 121, no. 4, pp. 804-813, 1999.

[117] S. W. Hong and A. E. Bergles, "Augmentation of laminar flow heat transfer in tubes by means of twisted-tape inserts," Journal of Heat Transfer, vol. 98, no. 2, pp. 251-256, 1976.

[118] J. J. Hwang and C. S. Cheng, "Augmented heat transfer in a triangular duct by using multiple swirling jets," Journal of Heat Transfer, vol. 121, no. 3, pp. 683-690, 1999.

[119] J. B. W. Kok, F. J. J. Rosendal, and J. J. H. Brouwers, "LDAmeasurements on swirling flows in tubes," in Proceedings of the 5th International Conference on Laser Anemometry, vol. 2052 of Proceedings of SPIE, pp. 721-728, August 1993.

[120] F. Kreith and D. Margolis, "Heat transfer and friction in turbulent vortex flow," Applied Scientific Research, vol. 8, no. 1, pp. 457-473, 1959.
[121] R. Kumar and T. Conover, "Flow visualization studies of a swirling flow in a cylinder," Experimental Thermal and Fluid Science, vol. 7, no. 3, pp. 254-262, 1993.

[122] H. Li and Y. Tomita, "Characteristics of swirling flow in a circular pipe," Journal of Fluids Engineering, vol. 116, no. 2, pp. 370-373, 1994.

[123] P. M. Ligrani, C. R. Hedlund, B. T. Babinchak, R. Thambu, H. K. Moon, and B. Glezer, "Flow phenomena in swirl chambers," Experiments in Fluids, vol. 24, no. 3, pp. 254-264, 1998.

[124] H.-K. Moon, T. O'Connell, and B. Glezer, "Heat transfer enhancement in a circular channel using lengthwise continuous tangential injection," in Proceedings of the International Heat Transfer Congress (IHTC '98), Seoul, South Korea, 1998.

[125] P. Papadopoulos, D. M. France, and W. J. Minkowycz, "Heat transfer to dispersed swirl flow of high-pressure water with low wall superheat," Experimental Heat Transfer, vol. 4, no. 2, pp. 153-169, 1991.

[126] R. Razgaitis and J. P. Holman, "A survey of heat transfer in confined swirling flows," in Future Energy Production Systems, Heat and Mass Transfer Processes, vol. 2, pp. 831-866, Academic Press, New York, NY, USA, 1976.

[127] W. F. J. Sampers, A. P. G. G. Lamers, and A. A. van Steenhoven, "Experimental and numerical analysis of a turbulent swirling flow in a tube," in Proceedings of the 3rd UK National Conference incorporating 1st European Conference on Thermal Sciences, vol. 2 of ICHEME Symposium Series no. 129, pp. 765-771, September 1992.

[128] W. L. Fu, L. M. Wright, and J. C. Han, "Rotational buoyancy effects on heat transfer in five different aspect-ratio rectangular channels with smooth walls and 45 degree ribbed walls," Journal of Heat Transfer, vol. 128, no. 11, pp. 1130-1141, 2006, ASME Paper GT 2005-68493.

[129] B. J. Belnap, J. A. Van Rij, and P. M. Ligrani, "A Reynolds analogy for real component surface roughness," International Journal of Heat and Mass Transfer, vol. 45, no. 15, pp. 3089-3099, 2002.

[130] J. A. Van Rij, B. J. Belnap, and P. M. Ligrani, "Analysis and experiments on three-dimensional, irregular surface roughness," Journal of Fluids Engineering, Transactions of the ASME, vol. 124, no. 3, pp. 671-677, 2002.

[131] D. L. Gee and R. L. Webb, "Forced convection heat transfer in helically Rib-roughened tubes," International Journal of Heat and Mass Transfer, vol. 23, no. 8, pp. 1127-1136, 1980.

[132] X. Chi, T. I.-P. Shihb, K. M. Bryden et al., "Effects of pin-fin height on flow and heat transfer in a rectangular duct," ASME Paper GT2011-46014, 2011.

[133] J. K. Ostanek and K. A. Thole, "Flow field measurements in a single row of low aspect ratio pin-fins," ASME Paper GT201145757, 2011.

[134] J. K. Ostanek and K. A. Thole, "Effects of varying steamwise and spanwise spacing in pin-fin arrays," ASME Paper GT2012-68127, 2012.

[135] X. Wang, X. Bai, J. Wu, R. Liu, D. Zhu, and G. Liao, "Numerical study on heat transfer characteristics in rectangular ducts with pin-fins," ASME Paper GT2011-45286, 2011.

[136] Y. Rao, C. Wan, and S. Zang, "Comparisons of flow friction and heat transfer performance in rectangular channels with pin findimple arrays," ASME Paper GT2010-22442, 2010.

[137] C. Bianchini, B. Facchini, S. Zacchi, F. Simonetti, and L. Tachi, "Numerical and experimental investigation of turning flow effects of innovative pin fin arrangements for trailing edge cooling configurations," ASME Paper GT2010-23536, 2010. 
[138] M. L. Busche, L. P. Moualeu, N. Chowdhury, C. Tang, and F. E. Ames, "Heat transfer and pressure drop measurements in high solidity pin fin cooling arrays with incremental replenishment," ASME Paper GT2012-69289, 2012.

[139] J. B. Cowan, D. K. Tafti, and A. Kohli, "Investigation of sand particle deposition and erosion characteristics within a short pin fin array," ASME Paper GT2010-22362, 2010.

[140] S. C. Siw, M. K. Chyu, T. I.-P. Shih, and M. A. Alvin, "Effects of pin detached space on heat transfer and from pin fin arrays," ASME Paper GT2010-23227, 2010.

[141] L. V. Tran, M. I. Valentino, A. Saha et al., "PIV study on the dimple mid-plane of a narrow rectangular channel with dimples applied to one wall," ASME Paper GT2011-46132, 2011.

[142] C. N. Jordan and L. M. Wright, "Heat transfer enhancement in a rectangular $(\mathrm{AR}=3: 1)$ channel with $\mathrm{V}$-shaped dimples," ASME Paper GT2011-46128, 2011.

[143] R. Amano, K. Guntur, J. M. Lucci, and Y. Ashitaka, "Study of flow through a stationary ribbed channel for blade cooling," ASME Paper GT2010-23031, 2010.

[144] J. E. Dees, G. A. Ledezma, A. K. Tolpadi, G. M. Lashowski, and D. G. Bogard, "Experimental measurements and computational predictions for an internally cooled simulated turbine vane with 90 degree Rib turbulators," ASME Paper GT2010-23004, 2010.

[145] T. Hagari, K. Ishida, K.-I. Takeishi, Y. Oda, and H. Kitada, "Investigation on heat transfer characteristics of a cooling channel With dense array of angled Rib turbulator," ASME Paper GT2012-68949, 2012.

[146] C. LeBlanc, S. V. Ekkad, T. Lambert, and V. Rajendran, "Detailed heat transfer distributions in engine similar cooling channels for a turbine rotor blade with different Rib orientations," ASME Paper GT2011-45254, 2011.

[147] G. Ledezma and R. Bunker, "The optimal distribution of chordwise Rib Fin arrays for blade Tip Cap underside cooling," ASME Paper GT2012-68432, 2012.

[148] J. Lei, M. Huh, and J.-C. Han, "Effect of rib spacing on heat transfer in a two pass rectangular channel $(\mathrm{AR}=2: 1)$ at high rotation numbers," ASME Paper GT2011-45926, 2011.

[149] J. Lei, S.-J. Li, J.-C. Han, L. Zhang, and H.-K. Moon, "Heat transfer in rotating multi-pass rectangular ribbed channel with and without a turning vane," ASME Paper GT2012-69139, 2012.

[150] S. Patil and D. Tafti, "Large-eddy simulation with zonal near wall treatment of flow and heat transfer in a ribbed duct for the internal cooling of turbine blades," ASME Paper GT2011-45749, 2011.

[151] B. V. N. Ramakumar, V. R. Kollati, S. Goswami, V. Kulkarni, J. S. Liu, and D. C. Crites, "Numerical validation of heat transfer augmentation factor in serpentine passages with ribbed walls," ASME Paper GT2010-22636, 2010.

[152] K. Saha and S. Acharya, "Effect of entrance geometry on heat transfer in a narrow ( $\mathrm{AR}=1: 4)$ rectangular two pass channel with smooth and ribbed walls," ASME Paper GT2011-46076, 2011.

[153] T. Salameh and B. Sunden, "Effects of ribs on internal blade-tip cooling," ASME Paper GT2011-45118, 2011.

[154] H. Zhang, J. Wang, X. Wu, and H. Lu, "A simplified approach to design transverse ribs which array alternately in rectangular channel," ASME Paper, no. GT2010-22562, 2010.

[155] T. Salameh and B. Sunden, "Comparison of continuous and truncated ribs on internal blade tip cooling," ASME Paper GT2012-68028, 2012.

[156] G. Xie, S. Li, W. Zhang, and B. Sundén, “Computational analysis of side-wall heat transfer of a turbine blade internal cooling passage with truncated ribs on opposite walls," ASME Paper GT2012-68073, 2012.

[157] J. Wang, H. Liu, M. Mao, X. Li, and Z. Zhang, "Numerical investigations of fluid flow and heat transfer performances of semiattached rib channel design," ASME Paper GT2010-22563, 2010.

[158] D. M. Lee, J. S. Park, D. H. Lee, B. S. Kim, and H. H. Cho, "Heat transfer characteristics of a non-rotating two-pass rectangular duct with various guide vanes in the tip turn region," ASME Paper GT2011-45962, 2011.

[159] B. Cukurel, C. Selcan, and T. Arts, "Film cooling extraction effects on the aero-thermal characteristics of rib roughened cooling channel flow," ASME Paper GT2012-68680, 2012.

[160] T. Guo, H. Zhu, and D. Xu, "Heat transfer investigation of the channels with Rib turbulators and double-row bleed holes," ASME Paper GT2011-46319, 2011.

[161] Y. Xing and B. Weigand, "Experimental investigation on staggered impingement heat transfer on a rib roughened plate with different crossflow schemes," ASME Paper GT2010-22043, 2010.

[162] F. Coletti, M. Scialanga, and T. Arts, "Experiemental investigation of conjugate heat transfer in a rib-roughened trailing edge channel with crossing-jets," ASME Paper GT2010-22432, 2010.

[163] F. Hoefler, S. Schueren, J. von Wolfersdorf, and S. Naik, "Heat transfer characteristics of an oblique jet impingement configuration in a passage with ribbed surfaces," ASME Paper GT2010-22288, 2010.

[164] Q. Yang, Y. Lin, C. Zhang, C.-J. Sung, and Q. Xu, "Cooling effectiveness of impingement/effusion cooling with and turbulence promoter ribs," ASME Paper GT2012-69209, 2012.

[165] J. Liu, J. Gao, and T. Gao, "An experimental investigation of heat transfer characteristics in a steam-cooled square channel with rib turbulators," ASME Paper GT2011-46134, 2011.

[166] L. Shui, J. Gao, L. Xu, and X. Wang, "Numerical investigation of heat transfer and flow characteristics in a steam-cooled square ribbed duct," ASME Paper GT2010-22407, 2010.

[167] T. S. Dhanasekaran and T. Wang, "Mist/air cooling in a twopass rectangular rotating channel with 45-deg angled Rib turbulators," ASME Paper GT2011-45954, 2011.

[168] R. S. Amano, K. S. Guntur, J. Martinez-Lucci, and S. Kumar, "Numerical comparison of heat transfer and pressure drop in gas turbine blade cooling channels with dimples and ribturbulators," ASME Paper GT2011-45277, 2011.

[169] L. Qui, H. Deng, and Z. Tao, "Effect of channel orientation in a rotating wedge-shaped cooling channel with pin fins and ribs," ASME Paper GT2012-68439, 2012.

[170] J. Lan, Y. Xie, and D. Zhang, "Heat transfer enhancement in a rectangular channel with the combination of ribs, dimples and protrusions," ASME Paper GT2011-46031, 2011.

[171] Z. Feng, Z. Dou, J. Wang, S. Ma, and Z. Zhang, "Numerical investigations of cooling enhancement with internal ribs and external coolant film," ASME Paper GT2012-68682, 2012.

[172] L. Wang, B. Sunden, A. Borg, and H. Abrahamsson, "Heat transfer in a channel under the effects of a shallow-angle jet impingement and a rib," ASME Paper GT2011-46333, 2011.

[173] S. Acharya, F. Zhou, J. Lagrone, G. Mahmood, and R. S. Bunker, "Latticework (Vortex) cooling effectiveness: rotating channel experiments," Journal of Turbomachinery, vol. 127, no. 3, pp. 471478, 2005, ASME Paper 2004-GT-53983.

[174] K. Takeishi, Y. Oda, Y. Miyake, and Y. Motoda, "Experimental and numerical study on the convective heat transfer and pressure loss in rectangular ducts with inclined pin-fins on a wavy endwall," ASME Paper 2012-69625, 2012. 
[175] Y. Motoda, K. Takeishi, Y. Oda, and Y. Miyake, "A study of the effect of angled pin fins on endwall heat transfer," in Proceedings of the International Conference on Power Engineering (ICOPE '09), Kobe, Japan, 2009.

[176] R. S. Bunker, J. C. Bailey, C.-P. Lee, and C. W. Stevens, "In-wall netweork (Mesh) cooling augmentation of gas turbine airfoils," ASME Paper 2004-54260, 2004.

[177] Y. Servouze and J. C. Sturgis, "Heat transfer and flow field measurements in a rib-roughened branch of a rotating two-pass duct," ASME Paper GT2003-38048, 2003.

[178] J. P. Bons and J. L. Kerrebrock, "Complementary velocity and heat transfer measurements in a rotating cooling passage with smooth walls," Journal of Turbomachinery, vol. 121, no. 4, pp. 651-662, 1999, ASME Paper 98-GT-464.

[179] L. M. Wright, W. L. Fu, and J. C. Han, "Influence of entrance geometry on heat transfer in rotating rectangular cooling channels ( $\mathrm{AR}=4: 1$ ) with angled ribs," Journal of Heat Transfer, vol. 127, no. 4, pp. 378-387, 2005.

[180] S. Acharya, D. E. Nikitopoulos, and P. Agarwal, "Heat/mass transfer in a 4:1 AR smooth and ribbed coolant passage with rotation in 90-degree and 45-degree orientations," ASME Paper GT2004-53928, 2004.

[181] D. H. Lee, D. H. Rhee, and H. H. Cho, "Heat transfer measurements in a rotating equilateral triangular channel with various rib arrangements," ASME Paper GT2006-90973, 2006.

[182] Y. H. Liu, L. M. Wright, W. L. Fu, and J. C. Han, "Rib spacing effect on heat transfer and pressure loss in a rotating twopass rectangular channel ( $\mathrm{AR}=1: 2)$ with 45-degree angled ribs," AIAA Journal of Thermophysics and Heat Transfer, vol. 21, no. 3, pp. 582-595, 2006, ASME Paper GT2006-90368.

[183] L. Al-Hadhrami, T. Griffith, and J. C. Han, "Heat transfer in twopass rotating rectangular channels $(\mathrm{AR}=2)$ with five different orientations of $45 \mathrm{deg}$ v-shaped rib turbulators," Journal of Heat Transfer, vol. 125, no. 2, pp. 232-242, 2003, AIAA Paper 20020789.

[184] L. Al-Hadhrami and J. C. Han, "Effect of rotation on heat transfer in two-pass square channels with five different orientations of $45^{\circ}$ angled rib turbulators," International Journal of Heat and Mass Transfer, vol. 46, no. 4, pp. 653-669, 2003.

[185] H. D. Chiang and H. L. Li, "Jet impingement and forced convection cooling experimental study in rotating turbine blades," ASME Paper GT2009-59795, 2009.

[186] W. L. Fu, L. M. Wright, and J. C. Han, "Heat transfer in two-pass rotating rectangular channels $(\mathrm{AR}=1: 2$ and $\mathrm{AR}=1: 4)$ with $45^{\circ}$ angled Rib turbulators," Journal of Turbomachinery, vol. 127, no. 1, pp. 164-174, 2004, ASME Paper GT2004-53261.

[187] W. L. Fu, L. M. Wright, and J. C. Han, "Heat transfer in twopass rotating rectangular channels $(\mathrm{AR}=1: 2$ and $\mathrm{AR}=1: 4)$ with smooth walls," Journal of Heat Transfer, vol. 127, no. 3, pp. 265$277,2005$.

[188] T. J. Hajek and A. W. Higgins, "Coolant passage heat transfer with rotation," NASA N88-11160, Lewis Research Center, Turbine Engine Hot Section Technology, 1985.

[189] T. J. Hajek, J. H. Wagner, and B. V. Johnson, "Coolant passage heat transfer with rotation,” NASA N89-17314, Lewis Research Center, Turbine Engine Hot Section Technology, 1986.

[190] J. C. Han, Y. M. Zhang, and K. Kalkuehler, "Uneven wall temperature effect on local heat transfer in a rotating two-pass square channel with smooth walls," Journal of Heat Transfer, vol. 115, no. 4, pp. 912-920, 1993.
[191] S. S. Hsieh and W. J. Liu, "Uneven wall heat flux effect on local heat transfer in rotating two-pass channels with two opposite ribbed walls," Journal of Heat Transfer, vol. 118, no. 4, pp. 864876, 1996.

[192] S. S. Hsieh, Y. S. Wang, and M. H. Chiang, "Local heat transfer and velocity measurements in a rotating ribbed twopass square channel with uneven wall temperatures," Journal of Heat Transfer, vol. 119, no. 4, pp. 843-848, 1997.

[193] M. Huh, Y. H. Liu, J. C. Han, and S. Chopra, "Effect of rib spacing on heat transfer in a two-pass rectangular channel $(\mathrm{AR}=1: 4)$ with a sharp entrance at high rotation numbers," ASME Paper GT2008-50311, 2008.

[194] G. J. Hwang, S. C. Tzeng, C. P. Mao, and C. Y. Soong, "Heat transfer in a radially rotating four-pass serpentine channel with staggered half-V rib turbulators," Journal of Heat Transfer, vol. 123, no. 1, pp. 39-50, 2001.

[195] H. Ito and K. Nanbu, "Flow in rotating straight pipes of circular cross section," Journal of Basic Engineering, vol. 93, no. 3, pp. 383-394, 1971.

[196] T. M. Liou and C. C. Chen, "Heat transfer in a rotating twopass smooth passage with a $180^{\circ}$ rectangular turn," International Journal of Heat and Mass Transfer, vol. 42, no. 2, pp. 231-247, 1998.

[197] T. M. Liou, C. C. Chen, and M. Y. Chen, "TLCT and LDV measurements of heat transfer and fluid flow in a rotating sharp turning duct," International Journal of Heat and Mass Transfer, vol. 44, no. 9, pp. 1777-1787, 2001.

[198] T. M. Liou, M. Y. Chen, and M. H. Tsai, "Fluid flow and heat transfer in a rotating two-pass square duct with in-line 90-deg ribs," Journal of Turbomachinery, vol. 124, no. 2, pp. 260-268, 2002, ASME Paper 2001-GT-185.

[199] T. M. Liou, Y. S. Hwang, and M. Y. Chen, "Heat transfer improvement by arranging detached ribs on suction surfaces of rotating internal coolant passages," International Journal of Heat and Mass Transfer, vol. 50, no. 11-12, pp. 2414-2424, 2007.

[200] Y. H. Liu, M. Huh, J. C. Han, and S. Chopra, "Heat transfer in a two-pass rectangular channel $(\mathrm{AR}=1: 4)$ under high rotation numbers," Journal of Heat Transfer, vol. 130, no. 8, Article ID 081701, 9 pages, 2008, ASME Paper GT2007-27067.

[201] S. Mochizuki, J. Takamura, S. Yamawaki, and W. J. Yang, "Heat transfer in serpentine flow passages with rotation," Journal of Turbomachinery, vol. 116, no. 1, pp. 133-140, 1994, ASME Paper 92-GT-190.

[202] J. H. Wagner, B. V. Johnson, R. A. Graziani, and F. C. Yeh, "Heat transfer in rotating serpentine passages with trips normal to the flow," NASA TM 103758, 1991.

[203] J. H. Wagner, B. V. Johnson, R. A. Graziani, and F. C. Yeh, "Heat transfer in rotating serpentine passages with trips normal to the flow," Journal of Turbomachinery, vol. 114, no. 4, pp. 847-857, 1991.

[204] J. H. Wagner, B. V. Johnson, and F. C. Kopper, "Heat transfer in rotating serpentine passages with smooth walls," Journal of Turbomachinery, vol. 113, no. 3, pp. 321-330, 1991, ASME Paper 90-GT-331.

[205] W. J. Yang, N. Zhang, and J. Chiou, "Local heat transfer in a rotating serpentine flow passage," Journal of Heat Transfer, vol. 114, no. 2, pp. 354-361, 1992.

[206] F. Zhou, J. Lagrone, and S. Acharya, "Internal cooling in 4:1 AR passages at high rotation numbers," Journal of Heat Transfer, vol. 129, no. 12, pp. 1666-1675, 2007. 
[207] G. J. Hwang, S. C. Tzeng, and C. P. Mao, "Heat transfer of compressed air flow in a spanwise rotating four-pass serpentine channel," Journal of Heat Transfer, vol. 121, no. 3, pp. 583-591, 1999.

[208] T. S. Griffith, L. Al-Hadhrami, and J. C. Han, "Heat transfer in rotating rectangular cooling channels $(\mathrm{AR}=4)$ with angled ribs," Journal of Heat Transfer, vol. 124, no. 4, pp. 617-625, 2002.

[209] T. S. Griffith, L. Al-Hadhrami, and J. C. Han, "Heat transfer in rotating rectangular cooling channels $(\mathrm{AR}=4)$ with dimples," Journal of Turbomachinery, vol. 125, no. 3, pp. 555-563, 2003, ASME Paper 2002-GT-30220.

[210] J. C. Han and Y. M. Zhang, "Effect of uneven wall temperature on local heat transfer in a rotating square channel with smooth walls and radial outward flow," Journal of Heat Transfer, vol. 114, no. 4, pp. 850-858, 1992.

[211] J. C. Han, Y. M. Zhang, and C. P. Lee, "Influence of surface heating condition on local heat transfer in a rotating square channel with smooth walls and radial outward flow," Journal of Turbomachinery, vol. 116, no. 1, pp. 149-158, 1994, ASME Paper 92-GT-188.

[212] E. Lee, L. M. Wright, and J. C. Han, "Heat transfer in rotating rectangular channels $(\mathrm{AR}=4: 1)$ with $\mathrm{V}$-shaped and angled rib turbulators with and without gaps," ASME Paper GT200338900, 2003.

[213] L. M. Wright, E. Lee, and J. C. Han, "Effect of rotation on heat transfer in rectangular channels with pin fins," Journal of Thermophysics and Heat Transfer, vol. 18, no. 2, pp. 263-272, 2004.

[214] N. Zhang, J. Chiou, S. Fann, and W. J. Yang, "Local heat transfer distribution in a rotating serpentine rib-roughened flow passage," Journal of Heat Transfer, vol. 115, no. 3, pp. 560567, 1993.

[215] G. J. Hwang and C. R. Kuo, "Experimental studies and correlations of convective heat transfer in a radially rotating serpentine passage," Journal of Heat Transfer, vol. 119, no. 3, pp. 460-466, 1997.

[216] K. M. Kim, Y. Y. Kim, D. H. Lee, D. H. Rhee, and H. H. Cho, "Influence of duct aspect ratio on heat/mass transfer in coolant passages with rotation," International Journal of Heat and Fluid Flow, vol. 28, no. 3, pp. 357-373, 2007.

[217] C. R. Kuo and G. J. Hwang, "Experimental studies and correlations of radially outward and inward air-flow heat transfer in a rotating square duct," Journal of Heat Transfer, vol. 118, no. 1, pp. 23-30, 1996.

[218] C. R. Kuo and G. J. Hwang, "Aspect ratio effect on convective heat transfer of radially outward flow in rotating rectangular ducts," International Journal of Rotating Machinery, vol. 1, no. 1, pp. 1-18, 1994.

[219] L. M. Wright, W. L. Fu, and J. C. Han, “Thermal performance of angled, $\mathrm{V}$-shaped, and $\mathrm{W}$-shaped rib turbulators in rotating rectangular cooling channels $(\mathrm{AR}=4: 1)$," Journal of Turbomachinery, vol. 126, no. 4, pp. 604-614, 2004, ASME Paper 2004GT-54073.

[220] S. W. Chang and W. D. Morris, "Heat transfer in a radially rotating square duct fitted with in-line transverse ribs," International Journal of Thermal Sciences, vol. 42, no. 3, pp. 267-282, 2003.

[221] S. W. Chang, T. L. Yang, T. M. Liou, and G. F. Hong, "Heat transfer of rotating rectangular duct with compound scaled roughness and V-ribs at high rotation numbers," International Journal of Thermal Sciences, vol. 48, no. 1, pp. 174-187, 2009.

[222] S. W. Chang, T. L. Yang, T. M. Liou, and H. G. Fang, "Heat transfer in rotating scale-roughened trapezoidal duct at high rotation numbers," Applied Thermal Engineering, vol. 29, no. 89, pp. 1682-1693, 2009.

[223] S. Dutta, J. C. Han, Y. Zhang, and C. P. Lee, "Local heat transfer in a rotating two-pass triangular duct with smooth walls," Journal of Turbomachinery, vol. 118, no. 3, pp. 435-443, 1996, ASME Paper 94-GT-337.

[224] S. P. Harasgama and W. D. Morris, "The influence of rotation on the heat transfer characteristics of circular, triangular, and square-sectioned coolant passages of gas turbine rotor blades," Journal of Turbomachinery, vol. 110, no. 1, pp. 44-50, 1988.

[225] S. S. Hsieh and Y. J. Hong, "Heat transfer coefficients in an orthogonally rotating duct with turbulators," Journal of Heat Transfer, vol. 117, no. 1, pp. 69-78, 1995.

[226] W. D. Morris and T. Ayhan, "Observations on the influence of rotation on heat transfer in the coolant channels of gas turbine rotor blades," Proceedings of the Institution of Mechanical Engineers, vol. 193, pp. 303-311, 1979.

[227] W. D. Morris and S. W. Chang, "An experimental study of heat transfer in a simulated turbine blade cooling passage," International Journal of Heat and Mass Transfer, vol. 40, no. 15, pp. 3703-3716, 1997.

[228] W. D. Morris and R. Salemi, "An attempt to uncouple the effect of Coriolis and buoyancy forces experimentally on heat transfer in smooth circular tubes that rotate in the orthogonal mode," Journal of Turbomachinery, vol. 114, no. 4, pp. 858-864, 1992, ASME Paper 91-GT-17.

[229] A. Murata, S. Mochizuki, and T. Takahashi, "Local heat transfer measurements of an orthogonally rotating square duct with angled rib turbulators," International Journal of Heat and Mass Transfer, vol. 42, no. 16, pp. 3047-3056, 1999.

[230] L. M. Wright, Y. H. Liu, J. C. Han, and S. Chopra, "Heat transfer in trailing edge, wedge-shaped cooling channels under high rotation numbers," Journal of Heat Transfer, vol. 130, no. 7, Article ID 071701, 11 pages, 2007, ASME Paper GT2007-27093.

[231] S. W. Chang, T. M. Liou, J. H. Hung, and W. H. Yeh, "Heat transfer in a radially rotating square-sectioned duct with two opposite walls roughened by 45 deg staggered ribs at high rotation numbers," Journal of Heat Transfer, vol. 129, no. 2, pp. 188-199, 2007.

[232] S. Dutta, J. C. Han, and C. P. Lee, "Experimental heat transfer in a rotating triangular duct: effect of model orientation," Journal of Heat Transfer, vol. 117, no. 4, pp. 1058-1061, 1995.

[233] T. M. Liou, S. W. Chang, J. H. Hung, and S. F. Chiou, "High rotation number heat transfer of a $45^{\circ}$ rib-roughened rectangular duct with two channel orientations," International Journal of Heat and Mass Transfer, vol. 50, no. 19-20, pp. 40634078, 2007.

[234] W. D. Morris and K. F. Rahmat-Abadi, "Convective heat transfer in rotating ribbed tubes," International Journal of Heat and Mass Transfer, vol. 39, no. 11, pp. 2253-2266, 1996.

[235] F. T. Willett and A. E. Bergles, "Heat transfer in rotating narrow rectangular pin-fin ducts," Experimental Thermal and Fluid Science, vol. 25, no. 7, pp. 573-582, 2002.

[236] S. W. Chang, T. L. Yang, and W. J. Wang, "Heat transfer in a rotating twin-pass trapezoidal-sectioned passage roughened by skewed ribs on two opposite walls," Heat Transfer Engineering, vol. 27, no. 10, pp. 63-79, 2006.

[237] W. D. Morris and G. Ghavami-Nasr, "Heat transfer measurements in rectangular channels with orthogonal mode rotation," Journal of Turbomachinery, vol. 113, no. 3, pp. 339-345, 1991, ASME Paper 90-GT-138. 
[238] S. Acharya, V. Eliades, and D. E. Nikitopoulos, "Heat transfer enhancements in rotating two-pass coolant channels with profiled ribs: part 1-average results," Journal of Turbomachinery, vol. 123, no. 1, pp. 97-106, 2001, ASME Paper 2000-GT-227.

[239] P. Agarwal, S. Acharya, and D. E. Nikitopoulos, "Heat transfer in 1:4 rectangular passages with rotation," Journal of Turbomachinery, vol. 125, no. 4, pp. 726-733, 2003.

[240] H. H. Cho, Y. Y. Kim, K. Kim, and D. H. Rhee, "Effects of rib arrangements and rotation speed on heat transfer in a two-pass duct," ASME Paper GT-2003-38609, 2003.

[241] H. H. Cho, S. Y. Lee, and D. H. Rhee, "Effects of cross ribs on heat/mass transfer in a two-pass rotating duct," Heat and Mass Transfer, vol. 40, no. 10, pp. 743-755, 2004.

[242] H. H. Cho, S. Y. Lee, J. H. Won, and D. H. Rhee, "Heat/mass transfer in a two-pass rotating rectangular duct with and without $70^{\circ}$-angled ribs," Heat and Mass Transfer, vol. 40, no. 6-7, pp. 467-475, 2004.

[243] K. M. Kim, D. H. Lee, and H. H. Cho, "Detailed measurement of heat/mass transfer and pressure drop in a rotating two-pass duct with transverse ribs," Heat and Mass Transfer, vol. 43, no. 8, pp. 801-815, 2007.

[244] C. W. Park and S. C. Lau, "Effect of channel orientation of local heat (mass) transfer distributions in a rotating two-pass square channel with smooth walls," Journal of Heat Transfer, vol. 120, no. 3, pp. 624-632, 1998.

[245] C. W. Park, S. C. Lau, and R. T. Kukreja, "Heat (mass) transfer in a rotating channel with ribs of various sizes on two walls," Journal of Thermophysics and Heat Transfer, vol. 12, no. 3, pp. 452-454, 1998.

[246] C. W. Park, S. C. Lau, and R. T. Kukreja, "Heat/mass transfer in a rotating two-pass channel with transverse ribs," Journal of Thermophysics and Heat Transfer, vol. 12, no. 1, pp. 80-86, 1998.

[247] C. W. Park, C. Yoon, and S. C. Lau, "Heat (mass) transfer in a diagonally oriented rotating two-pass channel with ribroughened walls," Journal of Heat Transfer, vol. 122, no. 1, pp. 208-211, 2000.

[248] S. W. Chang, T.-M. Liou, and T.-H. Lee, "Detailed heat transfer distributions and pressure drop measurements for a rotating parallelogram channel with radially outward flow," ASME Paper GT2011-45456, 2011.

[249] S.-Y. Hu, T. I.-P. Shih, M. K. Chyu et al., "Flow and heat transfer in the tip-turn region of a U-duct under rotating and nonrotating conditions," ASME Paper GT2011-46013, 2011.

[250] M. Huh, J. Lei, and J.-C. Han, "Influence of channel orientation on heat transfer in a two-pass smooth and ribbed rectangular channel (AR=2:1) under large rotation numbers," ASME Paper GT2010-22190, 2010.

[251] A. P. Rallabandi, Y.-H. Liu, and J.-C. Han, "Heat transfer in trailing edge wedge-shaped pin-fin channels with slot ejection under high rotation numbers," ASME Paper GT2010-22832, 2010.

[252] S. W. Chang, T.-M. Liou, and T.-H. Lee, "Heat transfer of rotating rectangular channel with diamond shaped pin-fin array at high rotation numbers," ASME Paper GT2012-68676, 2012.

[253] J. A. Lamont and S. V. Ekkad, "Effects of rotation on jet impingement channel heat transfer," ASME Paper GT201145744, 2011.

[254] M. A. Elyyan and D. K. Tafti, "Investigation of coriolis forces effect of flow structure and heat transfer distribution in a rotating dimpled channel," ASME Paper GT2010-22657, 2010.
[255] S. W. Chang, T.-M. Liou, and W. C. Chen, "Heat transfer in a rotating rectangular channel with two opposite walls roughened with spherical protrusions at high rotation numbers," ASME Paper GT2010-22609, 2010.

[256] L. Bonanni, C. Carcasci, B. Facchini, and L. Tarchi, "Experiemental survey on heat transfer in a trailing edge cooling system: effects of rotation in internal cooling ducts," ASME Paper GT2012-69638, 2012.

[257] D. Borello, G. Delibra, C. Bianchini, and A. Andreini, "Unsteady CFD analysis of turbulent flow and heat transfer in a gas turbine blade trailing edge subjected to rotation," ASME Paper GT201269903, 2012.

[258] M. Pascotto, A. Armellini, L. Casarsa, P. Giannattasio, and C. Mucignat, "Effects of rotation and channel orientation on the flow field inside a trailing edge internal cooling channel," ASME Paper GT2012-68050, 2012.

[259] M. Schroll, M. Elfert, and L. Lange, "Investigation of the effect of rotation on the flow in a two-pass cooling system with smooth and ribbed walls using PIV," ASME Paper GT2011-46427, 2011.

[260] M. Schüler, Dreher, H. -M, M. Elfert, S. O. Neumann, and B. Weigand, "Numerical predictions of the effect of rotation on fluid flow and heat transfer in an engine-similar two-pass internal cooling channel with smooth and ribbed walls," ASME Paper GT2010-22870, 2010.

[261] H. A. El-Husayni, M. E. Taslim, and D. M. Kercher, "Experimental heat transfer investigation of stationary and orthogonally rotating asymmetric and symmetric heated smooth and turbulated channels," Journal of Turbomachinery, vol. 116, no. 1, pp. 124-132, 1994, ASME Paper 92-GT-189.

[262] J. Guidez, "Study of the convective heat transfer in a rotating coolant channel," Journal of Turbomachinery, vol. 111, no. 1, pp. 43-50, 1989.

[263] T. J. Hajek, J. H. Wagner, and B. V. Johnson, "Coolant passage heat transfer with rotation," NASA, Lewis Research Center, Turbine Engine Hot Section Technology, 1987.

[264] E. Sethuraman, S. Acharya, and D. E. Nikitopoulos, "Mass/heat transfer in rotating, smooth, high-aspect ratio (4:1) coolant channels with curved walls," Journal of Turbomachinery, vol. 131, no. 2, Article ID 021002, 9 pages, 2009.

[265] F. T. Willett and A. E. Bergles, "Heat transfer in rotating narrow rectangular ducts with heated sides parallel to the r-z plane," Journal of Heat Transfer, vol. 124, no. 1, pp. 1-7, 2002.

[266] F. T. Willett and A. E. Bergles, "Heat transfer in rotating narrow rectangular ducts with heated sides oriented at $60^{\circ}$ to the $\mathrm{r}-\mathrm{z}$ plane," Journal of Turbomachinery, vol. 123, no. 2, pp. 288-295, 2001, ASME Paper 2000-GT-224.

[267] R. J. Clifford, W. D. Morris, and S. P. Harasgama, "An experimental study of local and mean heat transfer in a triangularsectioned duct rotating in the orthogonal mode," Journal of Engineering for Gas Turbines and Power, vol. 106, no. 3, pp. 661667, 1984.

[268] Y. H. Liu, M. Huh, J. C. Han, and H. K. Moon, "High rotation number effects on heat transfer in a triangular channel with $45^{\circ}$, inverted $45^{\circ}$, and $90^{\circ}$ ribs," ASME Paper GT2009-59216, 2009.

[269] Y. H. Liu, M. Huh, D. H. Rhee, J. C. Han, and H. K. Moon, "Heat transfer in leading edge, triangular shaped cooling channels with angled ribs under high rotation numbers," Journal of Turbomachinery, vol. 131, no. 4, Article ID 041017, 12 pages, 2009, ASME Paper GT2008-50344.

[270] Y. Mori, T. Fukada, and W. Nakayama, "Convective heat transfer in a rotating radial circular pipe (2nd report)," International 
Journal of Heat and Mass Transfer, vol. 14, no. 11, pp. 1807-1824, 1971.

[271] T. M. Liou, C. C. Chen, and M. Y. Chen, "Rotating effect on fluid flow in two smooth ducts Connected by a 180-degree bend," ASME Journal of Fluids Engineering, vol. 125, no. 1, pp. 138-148, 2003. 

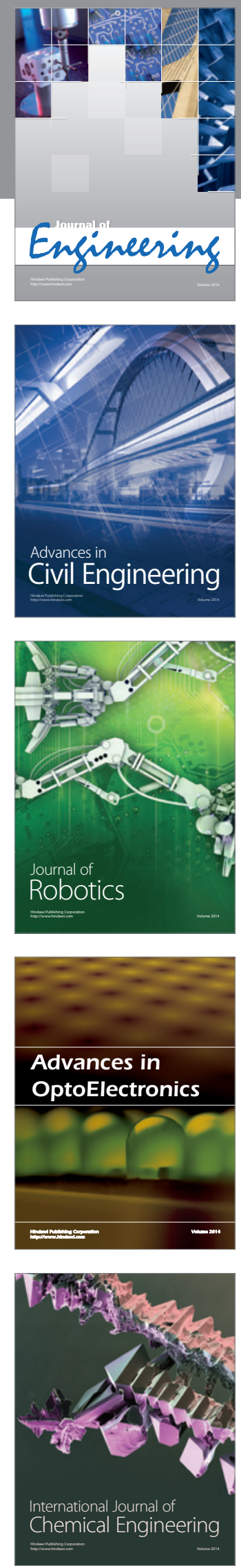

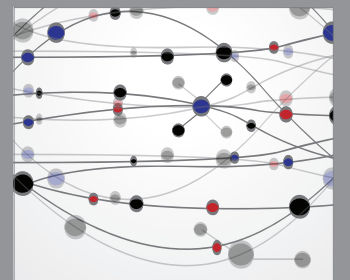

The Scientific World Journal
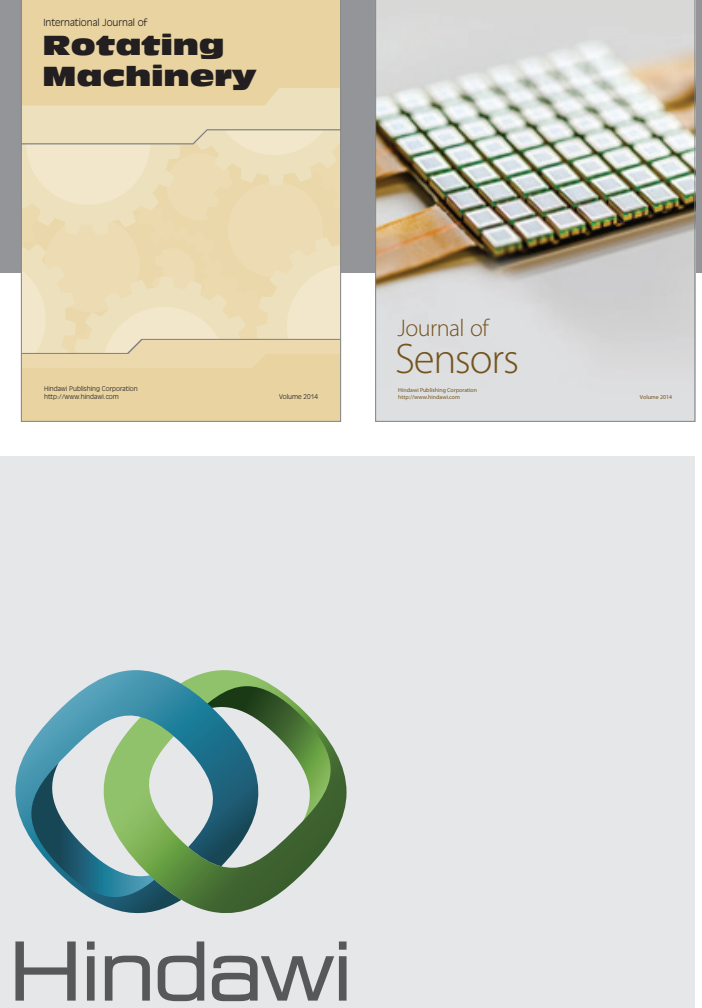

Submit your manuscripts at http://www.hindawi.com
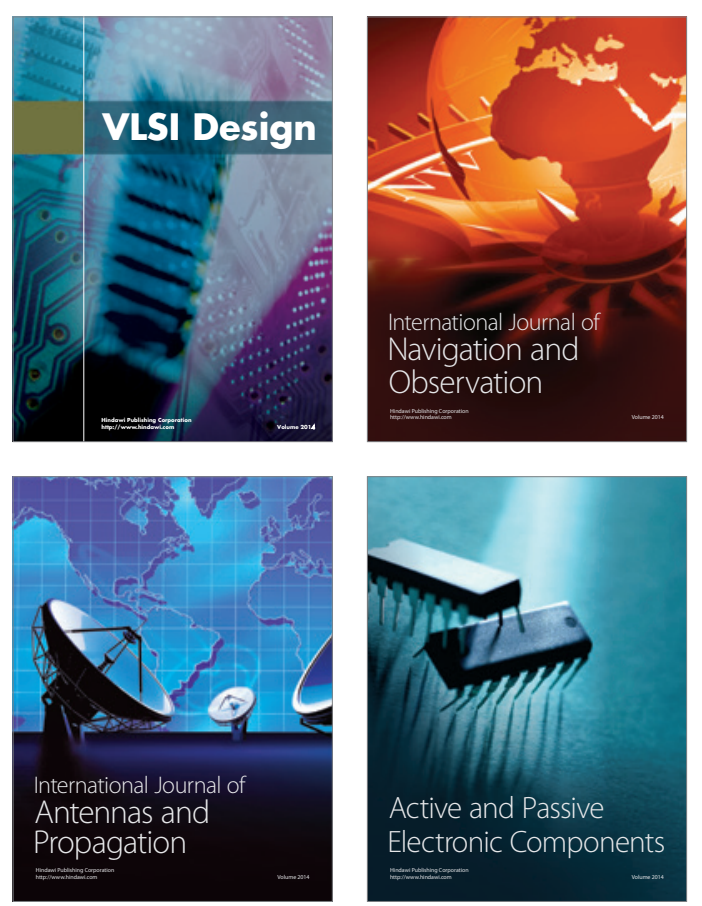
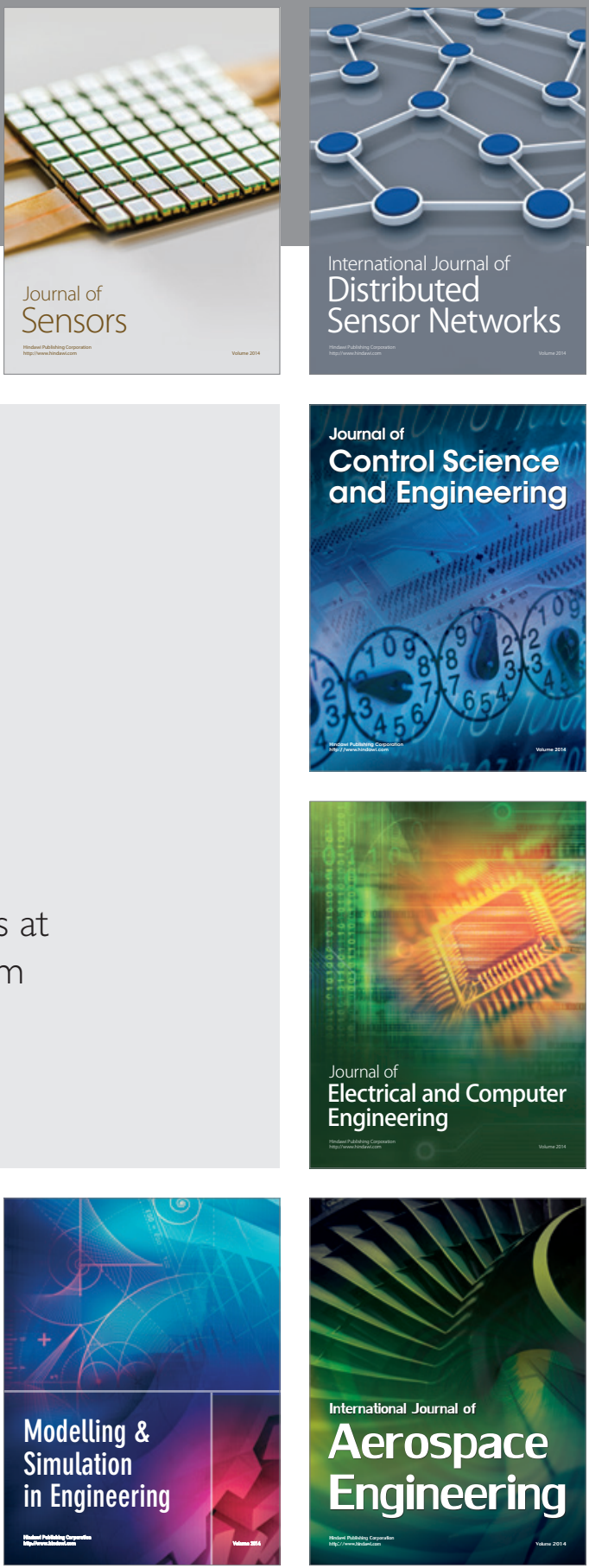

Journal of

Control Science

and Engineering
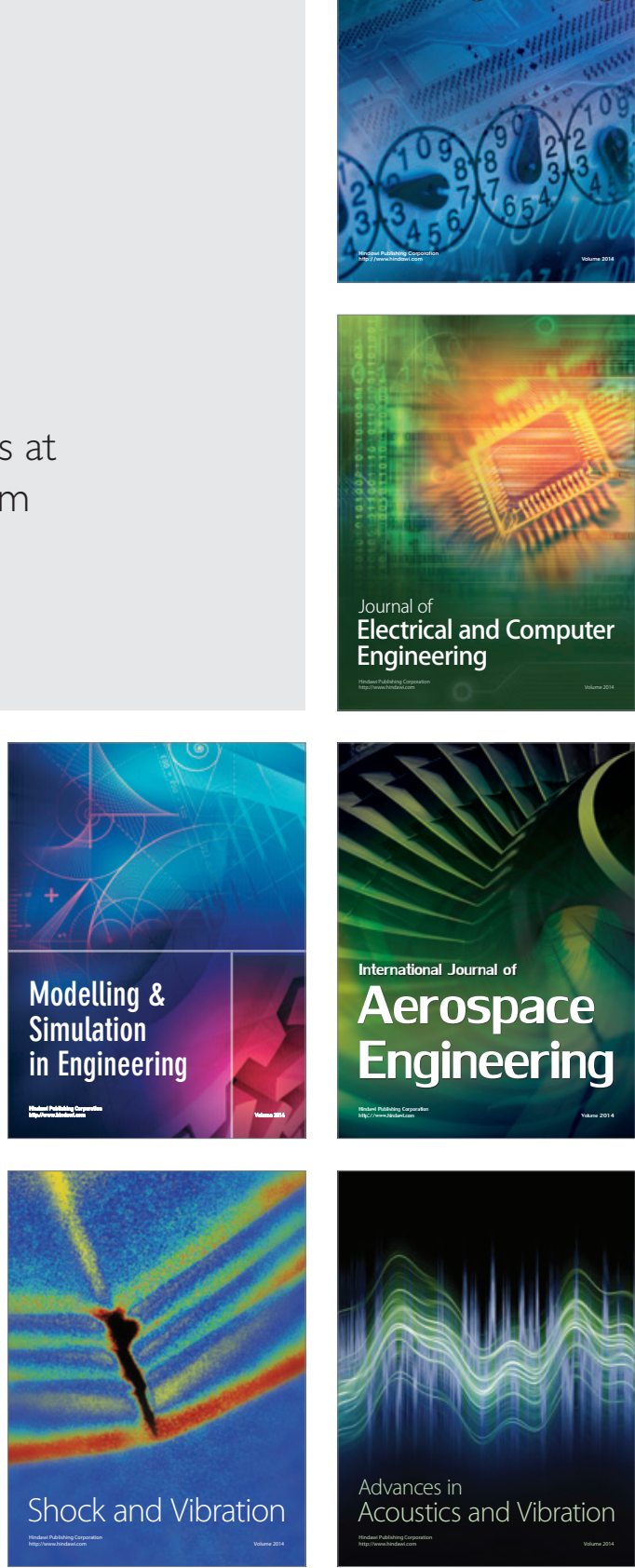